\title{
DECAY ESTIMATES ON SOLUTIONS OF THE LINEARIZED COMPRESSIBLE NAVIER-STOKES EQUATION AROUND A PARALLEL FLOW IN A CYLINDRICAL DOMAIN
}

\author{
Reika AOYAMA
}

(Received 19 June 2014 and revised 30 October 2014)

Abstract. This paper is concerned with the stability of a parallel flow of the compressible Navier-Stokes equation in a cylindrical domain. It is proved that the linearized semigroup around the parallel flow decays in the $L^{2}$-norm as a one-dimensional heat semigroup when the Reynolds and Mach numbers are sufficiently small. The proof is given by combining the energy method of Iooss-Padula and a variant of the Matsumura-Nishida energy method.

\section{Introduction}

We consider the system of equations for a barotropic motion of viscous compressible gas

$$
\begin{gathered}
\partial_{t} \rho+\operatorname{div}(\rho v)=0, \\
\rho\left(\partial_{t} v+v \cdot \nabla v\right)-\mu \Delta v-\left(\mu+\mu^{\prime}\right) \nabla \operatorname{div} v+\nabla P(\rho)=\rho g
\end{gathered}
$$

in a cylindrical domain $\Omega=D \times \mathbb{R}$ :

$$
\Omega=\left\{x=\left(x^{\prime}, x_{3}\right) ; x^{\prime}=\left(x_{1}, x_{2}\right) \in D, x_{3} \in \mathbb{R}\right\} .
$$

Here $D$ is a bounded and connected domain in $\mathbb{R}^{2}$ with a smooth boundary $\partial D ; \rho=\rho(x, t)$ and $v={ }^{\mathrm{T}}\left(v^{1}(x, t), v^{2}(x, t), v^{3}(x, t)\right)$ denote the unknown density and velocity at time $t \geq 0$ and position $x \in \Omega$, respectively; $P(\rho)$ is the pressure that is a smooth function of $\rho$ and satisfies

$$
P^{\prime}\left(\rho_{*}\right)>0
$$

for a given positive constant $\rho_{*} ; \mu$ and $\mu^{\prime}$ are the viscosity coefficients that are assumed to be constants satisfying

$$
\mu>0, \quad \frac{2}{3} \mu+\mu^{\prime} \geq 0
$$

and $g$ is an external force of the form $g={ }^{\mathrm{T}}\left(g^{1}\left(x^{\prime}\right), g^{2}\left(x^{\prime}\right), g^{3}\left(x^{\prime}\right)\right)$ with $g^{1}$ and $g^{2}$ satisfying

$$
\left(g^{1}\left(x^{\prime}\right), g^{2}\left(x^{\prime}\right)\right)=\left(\partial_{x_{1}} \Phi\left(x^{\prime}\right), \partial_{x_{2}} \Phi\left(x^{\prime}\right)\right),
$$

2010 Mathematics Subject Classification: Primary 35Q30; Secondary 76N15.

Keywords: compressible Navier-Stokes equation; parallel flow; cylindrical domain; linearized semigroup; decay estimates.

(C) 2015 Faculty of Mathematics, Kyushu University 
where $\Phi$ and $g^{3}$ are given smooth functions of $x^{\prime}$. Here and in what follows ${ }^{\mathrm{T}}$. stands for the transposition.

The system (1.1)-(1.2) is considered under the boundary condition

$$
\left.v\right|_{\partial D}=0
$$

and the initial condition

$$
\left.(\rho, v)\right|_{t=0}=\left(\rho_{0}, v_{0}\right) .
$$

One can see that problem (1.1)-(1.3) has the stationary solution $\bar{u}_{s}={ }^{\mathrm{T}}\left(\bar{\rho}_{s}, \bar{v}_{s}\right) ; \bar{\rho}_{s}$ is determined by

$$
\left\{\begin{array}{l}
\text { Const. }-\Phi\left(x^{\prime}\right)=\int_{\rho_{*}}^{\bar{\rho}_{s}} \frac{P^{\prime}(\eta)}{\eta} d \eta, \\
\int_{D} \bar{\rho}_{s}-\rho_{*} d x^{\prime}=0 ;
\end{array}\right.
$$

and $\bar{v}_{s}$ takes the form

$$
\bar{v}_{s}={ }^{\mathrm{T}}\left(0,0, \bar{v}_{s}^{3}\left(x^{\prime}\right)\right),
$$

where $\bar{v}_{s}^{3}\left(x^{\prime}\right)$ is the solution of

$$
\left\{\begin{array}{l}
-\Delta^{\prime} \bar{v}_{s}^{3}=\frac{1}{\mu} \bar{\rho}_{s} g^{3}, \\
\left.\bar{v}_{s}^{3}\right|_{\partial D}=0 .
\end{array}\right.
$$

Here $\Delta^{\prime}=\partial_{x_{1}}^{2}+\partial_{x_{2}}^{2}$. We are interested in the large time behavior of solutions to problem (1.1)-(1.4) when the initial value $\left.(\rho, v)\right|_{t=0}=\left(\rho_{0}, v_{0}\right)$ is sufficiently close to the stationary solution $\bar{u}_{s}={ }^{\mathrm{T}}\left(\bar{\rho}_{s}, \bar{v}_{s}\right)$. As a first step of the analysis, we study the linearized problem in this paper and establish decay estimates on solutions of the linearized equation around the parallel flow $\bar{u}_{s}$.

As for the asymptotic behavior of multi-dimensional compressible Navier-Stokes equations on unbounded domains, a lot of results have been obtained through the studies on the problems about global existence, stability, convergence rates and so on; see, for example, $[\mathbf{1}, \mathbf{3}, \mathbf{8}, \mathbf{1 2}-16,18]$ and references therein. Concerning the stability of parallel flows, in [9], the stability of a plane Poiseuille-type flow in an infinite layer of $\mathbb{R}^{n}$ was considered under the perturbations in some $L^{2}$-Sobolev space on the infinite layer. It was shown in [9] that the low-frequency part of the linearized semigroup behaves like a $(n-1)$-dimensional heat kernel and the high-frequency part decays exponentially as $t \rightarrow \infty$, provided that the Reynolds and Mach numbers are sufficiently small and the density of the parallel flow is sufficiently close to the given constant $\rho_{*}$. The nonlinear problem was studied by Kagei [7]; and it was proved that the stationary parallel flow is asymptotically stable under sufficiently small initial perturbations in some $L^{2}$-Sobolev space. Furthermore, the asymptotic behavior of the perturbation is described by a $(n-1)$-dimensional heat equation when $n \geq 3$. When $n=2$, the asymptotic behavior of the perturbation is no longer described by a linear equation but by a one-dimensional viscous Burgers equation.

As for the case of the cylindrical domain $\Omega$, Iooss and Padula [4] studied the linearized stability of a stationary parallel flow in $\Omega$ under the perturbations periodic in $x_{3}$. It was shown in [4] that the linearized operator generates a $C_{0}$-semigroup in $L^{2}$ on the basic periodicity cell 
under vanishing average condition for the density component. In particular, if the Reynolds number is suitably small, then the semigroup decays exponentially as time goes to infinity.

On the other hand, in [11], the stability of the rest state $\tilde{u}_{s}={ }^{\mathrm{T}}\left(\rho_{*}, 0\right)$ was considered under the perturbations in some $L^{2}$-Sobolev space on $\Omega$. It was shown in [11] that the solution of the linearized problem decays in $L^{2}(\Omega)$ in the order $t^{-1 / 4}$ and its asymptotic leading part is given by a solution of a one-dimensional heat equation. Furthermore, the asymptotic leading part of the perturbation is given by that for the linearized problem. (See also [5].)

The purpose of this paper is to extend the analysis for the rest state in [11] to the case of the general parallel flow in a cylindrical domain. We will establish decay estimates for solutions of the linearized problem for (1.1)-(1.4), which play an important role in the analysis of the nonlinear problem. To state our result more precisely, consider the nondimensional linearized problem:

$$
\partial_{t} u+L u=0,\left.\quad u\right|_{t=0}=u_{0}
$$

Here $u={ }^{\mathrm{T}}(\phi, w)={ }^{\mathrm{T}}\left(\gamma^{2}\left(\rho-\rho_{s}\right), v-v_{s}\right)$, and $L$ denotes the linearized operator on $L^{2}(\tilde{\Omega})$ defined by

$$
L=\left(\begin{array}{cc}
v_{s} \cdot \nabla & \gamma^{2} \operatorname{div}\left(\rho_{s} \cdot\right) \\
\nabla\left(\frac{\tilde{P}^{\prime}\left(\rho_{s}\right)}{\gamma^{2} \rho_{s}} \cdot\right) & -\frac{v}{\rho_{s}} \Delta I_{3}-\frac{\nu+v^{\prime}}{\rho_{s}} \nabla \operatorname{div}+v_{s} \cdot \nabla
\end{array}\right)+\left(\begin{array}{cc}
0 & 0 \\
\frac{v}{\gamma^{2} \rho_{s}^{2}} \Delta v_{s} I_{3} & \mathrm{~T}\left(\nabla v_{s}\right)
\end{array}\right),
$$

with domain

$$
D(L)=\left\{u={ }^{\mathrm{T}}(\phi, w) \in L^{2}(\tilde{\Omega}) ; w \in H_{0}^{1}(\tilde{\Omega}), L u \in L^{2}(\tilde{\Omega})\right\},
$$

where $\tilde{\Omega}, \tilde{D}, \tilde{\rho}, \tilde{v}, \rho_{s}, v_{s}$ and $\tilde{P}\left(\rho_{s}\right)$ are the non-dimensional form of $\Omega, D, \rho, v, \bar{\rho}_{s}, \bar{v}_{s}$ and $P\left(\bar{\rho}_{s}\right)$, respectively; $I_{3}$ denotes the $3 \times 3$ identity matrix; $v, v^{\prime}$ and $\gamma$ are some positive constants. We will prove that the linearized semigroup $e^{-t L}$ satisfies

$$
\left\|\partial_{x^{\prime}}^{k} \partial_{x_{3}}^{l} e^{-t L} u_{0}\right\|_{L^{2}(\tilde{\Omega})} \leq C\left\{t^{-1 / 4-l / 2}\left\|u_{0}\right\|_{L^{1}\left(\mathbb{R}: L^{2}(\tilde{D})\right)}+e^{-d t}\left\|u_{0}\right\|_{H^{1}(\tilde{\Omega})}\right\}
$$

for $t \geq 0$ and $0 \leq k+l \leq 1$, provided that the Reynolds number $\operatorname{Re}=1 / v$ and Mach number $\mathrm{Ma}=1 / \gamma$ are sufficiently small and that $\bar{\rho}_{s}$ is sufficiently close to $\rho_{*}$.

To prove (1.6), we consider the Fourier transform of (1.5) in $x_{3} \in \mathbb{R}$ which is written as

$$
\partial_{t} \widehat{u}+\widehat{L} \widehat{u}_{\xi}=0,\left.\quad \widehat{u}\right|_{t=0}=\widehat{u}_{0},
$$

where $\xi \in \mathbb{R}$ denotes the dual variable. The operator $\widehat{L}_{\xi}$ has different properties in the cases $|\xi| \ll 1$ and $|\xi| \gg 1$. We thus decompose the semigroup $e^{-t L}$ into two parts: $e^{-t L}=$ $\mathcal{F}^{-1}\left(e^{-t \widehat{L}_{\xi}}|| \xi \mid \leq 1\right)+\mathcal{F}^{-1}\left(e^{-t \widehat{L}_{\xi}}|| \xi \mid>1\right)$, where $\mathcal{F}^{-1}$ denotes the inverse Fourier transform. As for the low-frequency part, we take a new approach. A straightforward application of the arguments in $[\mathbf{9}, \mathbf{1 1}]$ seems to yield a more restrictive smallness conditions for the Reynolds and Mach numbers. To overcome this, we combine the arguments in $[\mathbf{9 , 1 1}]$ and the energy method in [4]. As in [9, 11], we decompose the low-frequency part of the semigroup according to the spectral properties of the linearized operator with zero frequency. The decay estimate for the $L^{2}$ norm is then established with the aid of the energy method in [4] applied to the decomposed system. Based on the decay estimate for $L^{2}$ norm, we obtain the estimate 
for the $L^{2}$ norm of the derivatives. We note that this approach also enables us to improve the decay estimate in $\left[\mathbf{9}, \mathbf{1 0}\right.$, Theorem 3.2] which is the one with $t^{-1 / 4}\left\|u_{0}\right\|_{L^{1}\left(\mathbb{R} ; L^{2}(\tilde{D})\right)}$ in (1.6) replaced by $t^{-1 / 4}\left\|u_{0}\right\|_{L^{1}\left(\mathbb{R} ; H^{1}(\tilde{D}) \times L^{2}(\tilde{D})\right)}$. On the other hand, in the case of the high-frequency part, we employ the Fourier-transformed version of Matsumura-Nishida's energy method as in $[9,11]$.

This paper is organized as follows. In Section 2 we first rewrite the problem into the system of equations in a non-dimensional form and then present the existence of a stationary solution of parallel flow type. We state our main results in Section 3. We derive the decay estimate of the low-frequency part in Section 4, and the high-frequency part in Section 5.

\section{Stationary solution and formulation of the problem}

We first rewrite the problem into the one in the non-dimensional form. We introduce the following non-dimensional variables:

$$
\begin{gathered}
x=\ell \tilde{x}, \quad v=V \tilde{v}, \quad \rho=\rho_{*} \tilde{\rho}, \quad t=\frac{\ell}{V} \tilde{t}, \\
P=\rho_{*} V^{2} \tilde{P}, \quad \Phi=\frac{V^{2}}{\ell} \tilde{\Phi}, \quad g^{3}=\frac{V^{2}}{\ell} \tilde{g}^{3}, \\
V=\left|\bar{v}_{s}^{3}\right|_{C_{*}^{3}(D)}=\sum_{k=0}^{3} \sup _{x^{\prime} \in D} \ell^{k}\left|\partial_{x^{\prime}}^{k} \bar{v}_{s}^{3}\left(x^{\prime}\right)\right|, \quad \ell=\left(\int_{D} d x^{\prime}\right)^{1 / 2} .
\end{gathered}
$$

The problem (1.1)-(1.3) is then transformed into the following non-dimensional problem on $\tilde{\Omega}=\tilde{D} \times \mathbb{R}:$

$$
\begin{gathered}
\partial_{\tilde{t}} \tilde{\rho}+\operatorname{div}_{\tilde{x}}(\tilde{\rho} \tilde{v})=0, \quad \tilde{t}>0, \\
\tilde{\rho}\left(\partial_{\tilde{t}} \tilde{v}+\tilde{v} \cdot \nabla_{\tilde{x}} \tilde{v}\right)-v \Delta_{\tilde{x}} \tilde{v}-\left(\nu+v^{\prime}\right) \nabla_{\tilde{x}} \operatorname{div}_{\tilde{x}} \tilde{v}+\tilde{P}^{\prime}(\tilde{\rho}) \nabla_{\tilde{x}} \tilde{\rho}=\tilde{\rho} \tilde{g}, \\
\left.\tilde{v}\right|_{\partial \tilde{D}}=0, \\
\left.(\tilde{\rho}, \tilde{v})\right|_{\tilde{t}=0}=\left(\tilde{\rho}_{0}, \tilde{v}_{0}\right) .
\end{gathered}
$$

Here $\tilde{D}$ is a bounded and connected domain in $\mathbb{R}^{2} ; \tilde{g}={ }^{\mathrm{T}}\left(\partial_{\tilde{x}_{1}} \tilde{\Phi}, \partial_{\tilde{x}_{2}} \tilde{\Phi}, \tilde{g}^{3}\right)$; and $v$ and $v^{\prime}$ are non-dimensional parameters:

$$
v=\frac{\mu}{\rho_{*} \ell V}, \quad v^{\prime}=\frac{\mu^{\prime}}{\rho_{*} \ell V} .
$$

We also introduce a parameter $\gamma$ :

$$
\gamma=\sqrt{\tilde{P}^{\prime}(1)}=\frac{\sqrt{P^{\prime}\left(\rho_{*}\right)}}{V} .
$$

In what follows, for simplicity, we omit tilde of $\tilde{x}, \tilde{t}, \tilde{v}, \tilde{\rho}, \tilde{g}, \tilde{P}, \tilde{\Phi}, \tilde{D}$ and $\tilde{\Omega}$ and write them as $x, t, v, \rho, g, P, \Phi, D$ and $\Omega$.

We next introduce some notation which will be used throughout the paper. For a domain $X$ and $1 \leq p \leq \infty$ we denote by $L^{p}(X)$ the usual Lebesgue space on $X$ and its norm is denoted by $\|\cdot\|_{L^{p}(X)}$. Let $m$ be a non-negative integer. Here $H^{m}(X)$ denotes the $m$ th-order 
$L^{2}$ Sobolev space on $X$ with norm $\|\cdot\|_{H^{m}(X)}$ and $C_{0}^{m}(X)$ stands for the set of all $C^{m}$ functions which have compact support in $X$. We denote by $H_{0}^{m}(X)$ the completion of $C_{0}^{m}(X)$ in $H^{m}(X)$.

We simply denote by $L^{p}(X)$ (respectively $H^{m}(X)$ ) the set of all vector fields $w=$ $\mathrm{T}\left(w^{1}, w^{2}, w^{3}\right)$ on $X$ and its norm is denoted by $\|\cdot\|_{L^{p}(X)}$ (respectively $\left.\|\cdot\|_{H^{m}(X)}\right)$. For $u={ }^{\mathrm{T}}(\phi, w)$ with $\phi \in H^{k}(X)$ and $w={ }^{\mathrm{T}}\left(w^{1}, w^{2}, w^{3}\right) \in H^{m}(X)$, we define $\|u\|_{H^{k}(X) \times H^{m}(X)}$ by $\|u\|_{H^{k}(X) \times H^{m}(X)}=\|\phi\|_{H^{k}(X)}+\|w\|_{H^{m}(X)}$.

In the case $X=\Omega$ we abbreviate $L^{p}(\Omega)$ (respectively $H^{m}(\Omega)$ ) as $L^{p}$ (respectively $H^{m}$ ). In particular, the norm $\|\cdot\|_{L^{p}(\Omega)}=\|\cdot\|_{L^{p}}$ (respectively $\left.\|\cdot\|_{H^{m}}\right)$.

In the case $X=D$ we denote the norm of $L^{p}(D)$ (respectively $H^{m}(D)$ ) by $|\cdot|_{p}$ (respectively $|\cdot|_{H^{m}}$ ). We denote the inner product of $L^{2}(D)$ by

$$
(f, g)=\int_{D} f\left(x^{\prime}\right) \overline{g\left(x^{\prime}\right)} d x^{\prime}, \quad f, g \in L^{2}(D) .
$$

Here $\bar{g}$ denotes the complex conjugate of $g$. For $u_{j}={ }^{\mathrm{T}}\left(\phi_{j}, w_{j}\right)(j=1,2)$, we also define a weighted inner product $\left\langle u_{1}, u_{2}\right\rangle$ by

$$
\left\langle u_{1}, u_{2}\right\rangle=\frac{1}{\gamma^{2}} \int_{D} \phi_{1} \bar{\phi}_{2} \frac{P^{\prime}\left(\rho_{s}\right)}{\gamma^{2} \rho_{s}} d x^{\prime}+\int_{D} w_{1} \cdot \bar{w}_{2} \rho_{s} d x^{\prime},
$$

where $\rho_{s}=\rho_{s}\left(x^{\prime}\right)$ is the density of the parallel flow $u_{s}$. As will be seen in Proposition 2.1 below, $\rho_{s}$ and $P^{\prime}\left(\rho_{s}\left(x^{\prime}\right)\right) / \rho_{s}\left(x^{\prime}\right)$ are strictly positive in $D$.

For $f \in L^{1}(D)$ we denote the mean value of $f$ in $D$ by $\langle f\rangle$ :

$$
\langle f\rangle=(f, 1)=\int_{D} f d x^{\prime} .
$$

For $u={ }^{\mathrm{T}}(\phi, w) \in L^{1}(D)$ with $w={ }^{\mathrm{T}}\left(w^{1}, w^{2}, w^{3}\right)$ we define $\langle u\rangle$ by

$$
\langle u\rangle=\langle\phi\rangle+\left\langle w_{1}\right\rangle+\left\langle w_{2}\right\rangle+\left\langle w_{3}\right\rangle .
$$

We often write $x \in \Omega$ as

$$
x={ }^{\mathrm{T}}\left(x^{\prime}, x_{3}\right), \quad x^{\prime}={ }^{\mathrm{T}}\left(x_{1}, x_{2}\right) \in D .
$$

Partial derivatives of a function $u$ in $x, x^{\prime}, x_{3}$ and $t$ are denoted by $\partial_{x} u, \partial_{x^{\prime}} u, \partial_{x_{3}} u$ and $\partial_{t} u$. We also write higher-order partial derivatives of $u$ in $x$ as $\partial_{x}^{k} u=\left(\partial_{x}^{\alpha} u ;|\alpha|=k\right)$.

We denote the $n \times n$ identity matrix by $I_{n}$. We define $4 \times 4$ diagonal matrices $Q_{0}, \tilde{Q}$, $Q^{\prime}$ and $Q_{3}$ by

$$
\begin{array}{ll}
Q_{0}=\operatorname{diag}(1,0,0,0), & \tilde{Q}=\operatorname{diag}(0,1,1,1), \\
Q^{\prime}=\operatorname{diag}(0,1,1,0), & Q_{3}=\operatorname{diag}(0,0,0,1) .
\end{array}
$$

We then have, for $u={ }^{\mathrm{T}}(\phi, w)$ with $w={ }^{\mathrm{T}}\left(w^{1}, w^{2}, w^{3}\right)$,

$$
Q_{0} u=\left(\begin{array}{c}
\phi \\
0
\end{array}\right), \quad \tilde{Q} u=\left(\begin{array}{c}
0 \\
w
\end{array}\right), \quad Q^{\prime} u=\left(\begin{array}{c}
0 \\
w^{1} \\
w^{2} \\
0
\end{array}\right), \quad Q_{3} u=\left(\begin{array}{c}
0 \\
0 \\
0 \\
w^{3}
\end{array}\right) .
$$


For a function $f=f\left(x_{3}\right)\left(x_{3} \in \mathbb{R}\right)$, we denote its Fourier transform by $\widehat{f}$ or $\mathcal{F}[f]$ :

$$
\widehat{f}(\xi)=\mathcal{F}[f](\xi)=\int_{\mathbb{R}} f\left(x_{3}\right) e^{-i \xi x_{3}} d x_{3}, \quad \xi \in \mathbb{R} .
$$

The inverse Fourier transform is denoted by $\mathcal{F}^{-1}$ :

$$
\mathcal{F}^{-1}[f]\left(x_{3}\right)=(2 \pi)^{-1} \int_{\mathbb{R}} f(\xi) e^{i \xi x_{3}} d \xi, \quad x_{3} \in \mathbb{R} .
$$

Let us state the existence of a stationary solution of Poiseuille flow.

Proposition 2.1. For $\Phi \in C^{3}(\bar{D})$ and $g^{3} \in H^{3}(D)$, there exists a positive constant $\delta_{0}$ such that if $|\Phi|_{C^{3}} \leq \delta_{0}$, then (2.1)-(2.3) has a stationary solution $u_{s}={ }^{\mathrm{T}}\left(\rho_{s}, v_{s}\right) \in C^{3}(\bar{D})$. Here $\rho_{s}$ is determined by

$$
\left\{\begin{array}{l}
\text { Const. }-\Phi\left(x^{\prime}\right)=\int_{1}^{\rho_{s}\left(x^{\prime}\right)} \frac{P^{\prime}(\eta)}{\eta} d \eta, \\
\int_{D} \rho_{s} d x^{\prime}=1, \quad \rho_{1}<\rho_{s}\left(x^{\prime}\right)<\rho_{2} \quad\left(\rho_{1}<1<\rho_{2}\right),
\end{array}\right.
$$

and $v_{s}$ is a function of the form $v_{s}={ }^{\mathrm{T}}\left(0,0, v_{s}^{3}\right)$ with $v_{s}^{3}=v_{s}^{3}\left(x^{\prime}\right)$ being the solution of

$$
\left\{\begin{array}{l}
-\Delta^{\prime} v_{s}^{3}=\frac{1}{v} \rho_{s} g^{3} \\
\left.v_{s}^{3}\right|_{\partial D}=0
\end{array}\right.
$$

Furthermore, $u_{s}={ }^{\mathrm{T}}\left(\rho_{s}, v_{s}\right)$ satisfies the estimates:

$$
\begin{gathered}
\left|\rho_{s}\left(x^{\prime}\right)-1\right|_{C^{3}} \leq C|\Phi|_{C^{3}}\left(1+|\Phi|_{C^{3}}\right)^{3}, \\
\left|v_{s}^{3}\right|_{C^{3}} \leq C\left|v_{s}^{3}\right|_{H^{5}} \leq C|\Phi|_{C^{3}}\left(1+|\Phi|_{C^{3}}\right)^{3}\left|g^{3}\right|_{H^{3}} .
\end{gathered}
$$

Proposition 2.1 can be proved in a similar manner to the proof of [17, Lemma 2.1]. We omit the proof.

From now on we simply denote $v+v^{\prime}$ by $\tilde{v}$ :

$$
\tilde{v}=v+v^{\prime} .
$$

Setting $\rho=\rho_{s}+\gamma^{-2} \phi$ and $v=v_{s}+w$ in (2.1)-(2.4), we arrive at the initial boundary value problem for the disturbance $u={ }^{\mathrm{T}}(\phi, w)$ that is written as follows:

$$
\begin{gathered}
\partial_{t} \phi+v_{s}^{3} \partial_{x_{3}} \phi+\gamma^{2} \operatorname{div}\left(\rho_{s} w\right)=f^{0}(\phi, w), \\
\partial_{t} w-\frac{v}{\rho_{s}} \Delta w-\frac{\tilde{v}}{\rho_{s}} \nabla \operatorname{div} w+\nabla\left(\frac{P^{\prime}\left(\rho_{s}\right)}{\gamma^{2} \rho_{s}} \phi\right) \\
+\frac{v}{\gamma^{2} \rho_{s}^{2}} \Delta^{\prime} v_{s}^{3} \phi e_{3}+v_{s}^{3} \partial_{x_{3}} w+\left(w^{\prime} \cdot \nabla^{\prime} v_{s}^{3}\right) e_{3}=f(\phi, w), \\
\left.w\right|_{\partial \Omega}=0, \\
\left.(\phi, w)\right|_{t=0}=\left(\phi_{0}, w_{0}\right) .
\end{gathered}
$$


Here $e_{3}={ }^{\mathrm{T}}(0,0,1) \in \mathbb{R}^{3}$ and $\nabla^{\prime}={ }^{\mathrm{T}}\left(\partial_{x_{1}}, \partial_{x_{2}}\right)$;

$$
\begin{gathered}
f^{0}(\phi, w)=-\operatorname{div}(\phi w), \\
f(\phi, w)=-w \cdot \nabla w+\frac{v \phi}{\left(\phi+\gamma^{2} \rho_{s}\right) \rho_{s}}\left(-\Delta w+\frac{1}{\gamma^{2} \rho_{s}} \Delta^{\prime} v_{s} \phi\right)-\frac{\tilde{v} \phi}{\left(\phi+\gamma^{2} \rho_{s}\right) \rho_{s}} \nabla \operatorname{div} w \\
+\frac{\phi}{\gamma^{2} \rho_{s}} \nabla\left(\frac{P^{\prime}\left(\rho_{s}\right) \phi}{\gamma^{2} \rho_{s}}\right)-\frac{1}{2 \gamma^{4} \rho_{s}} \nabla\left(P^{\prime \prime}\left(\rho_{s}\right) \phi^{2}\right)+\tilde{P}_{3}\left(\rho_{s}, \phi, \partial_{x^{\prime}} \phi\right),
\end{gathered}
$$

and

$$
\begin{aligned}
\tilde{P}_{3}\left(\rho_{s}, \phi, \partial_{x^{\prime}} \phi\right) \\
=\frac{\phi^{3}}{\gamma^{4}\left(\phi+\gamma^{2} \rho_{s}\right) \rho_{s}^{3}} \nabla P\left(\rho_{s}\right)-\frac{1}{2 \gamma^{6} \rho_{s}} \nabla\left(\phi^{3} P_{3}\left(\rho_{s}, \phi\right)\right) \\
\quad+\frac{\phi}{2 \gamma^{6} \rho_{s}^{2}} \nabla\left(P^{\prime \prime}\left(\rho_{s}\right) \phi^{2}+\frac{1}{\gamma^{2}} \phi^{3} P_{3}\left(\rho_{s}, \phi\right)\right) \\
\quad-\frac{\phi^{2}}{\gamma^{2}\left(\phi+\gamma^{2} \rho_{s}\right) \rho_{s}^{2}} \nabla\left(\frac{1}{\gamma^{2}} P^{\prime}\left(\rho_{s}\right) \phi+\frac{1}{2 \gamma^{4}} P^{\prime \prime}\left(\rho_{s}\right) \phi^{2}+\frac{1}{2 \gamma^{6}} \phi^{3} P_{3}\left(\rho_{s}, \phi\right)\right),
\end{aligned}
$$

with

$$
P_{3}\left(\rho_{s}, \phi\right)=\int_{0}^{1}(1-\theta)^{2} P^{\prime \prime \prime}\left(\rho_{s}+\theta \gamma^{-2} \phi\right) d \theta .
$$

Our main concern in this paper is decay estimates of solutions to the linearized problem, i.e. problem (2.5)-(2.8) with $f^{0}(\phi, w)=0$ and $f(\phi, w)=0$.

\section{Main results}

Let us consider the linearized problem

$$
\partial_{t} u+L u=0, \quad u={ }^{\mathrm{T}}(\phi, w),\left.\quad w\right|_{\partial D}=0,\left.\quad u\right|_{t=0}=u_{0} .
$$

Here $L$ is the operator on $L^{2}(\Omega)$ defined by

$$
\begin{aligned}
L & =\left(\begin{array}{cc}
v_{s} \cdot \nabla & \gamma^{2} \operatorname{div}\left(\rho_{s} \cdot\right) \\
\nabla\left(\frac{P^{\prime}\left(\rho_{s}\right)}{\gamma^{2} \rho_{s}} \cdot\right) & -\frac{v}{\rho_{s}} \Delta I_{3}-\frac{v+v^{\prime}}{\rho_{s}} \nabla \operatorname{div}+v_{s} \cdot \nabla
\end{array}\right)+\left(\begin{array}{cc}
0 & 0 \\
\frac{v}{\gamma^{2} \rho_{s}^{2}} \Delta^{\prime} v_{s} & e_{3} \otimes\left(\nabla v_{s}^{3}\right)
\end{array}\right) \\
& \equiv L_{1}+L_{2}
\end{aligned}
$$

with domain

$$
D(L)=\left\{u={ }^{\mathrm{T}}(\phi, w) \in L^{2}(\Omega) ; w \in H_{0}^{1}(\Omega), L u \in L^{2}(\Omega)\right\} .
$$

Here, for $\mathbf{a}={ }^{\mathrm{T}}\left(a_{1}, a_{2}, a_{3}\right)$ and $\mathbf{b}={ }^{\mathrm{T}}\left(b_{1}, b_{2}, b_{3}\right)$, we denote the $3 \times 3$ matrix $\left(a_{i} b_{j}\right)$ by $\mathbf{a} \otimes \mathbf{b}$.

In a similar manner to that in [4], one can show that $-L_{1}$ generates a $C_{0}$-semigroup on $L^{2}(\Omega)$. Since $\left\|L_{2} u\right\|_{2} \leq C\|u\|_{2}$, it follows from the standard perturbation theory that $-L$ generates a $C_{0}$-semigroup $e^{-t L}$ on $L^{2}(\Omega)$. It is not difficult to prove that if 
$u_{0} \in H^{1}(\Omega) \times H_{0}^{1}(\Omega)$, then

$$
\begin{aligned}
e^{-t L} u_{0} & \in C\left([0, T] ; H^{1}(\Omega) \times H_{0}^{1}(\Omega)\right), \\
Q_{0} e^{-t L} u_{0} & \in H^{1}\left(0, T ; L^{2}(\Omega)\right), \\
\tilde{Q} e^{-t L} u_{0} & \in L^{2}\left(0, T ; H^{2}(\Omega)\right) \cap H^{1}\left(0, T ; L^{2}(\Omega)\right)
\end{aligned}
$$

for all $T>0$.

In what follows we set

$$
\omega=\left\|\rho_{s}-1\right\|_{C^{3}} .
$$

The main results of this paper are stated as follows.

THEOREM 3.1. Suppose that $u_{0}={ }^{\mathrm{T}}\left(\phi_{0}, w_{0}\right) \in\left(H^{1}(\Omega) \times H_{0}^{1}(\Omega)\right) \cap L^{1}(\Omega)$. There exist positive constants $v_{0}, \gamma_{0}$ and $\omega_{0}$ such that if $v \geq v_{0}, \gamma^{2} /\left(2 v+v^{\prime}\right) \geq \gamma_{0}$ and $\left(2 v+v^{\prime}\right) \omega / v \leq$ $\omega_{0}$, then the estimate

$$
\left\|\partial_{x^{\prime}}^{k} \partial_{x_{3}}^{l} e^{-t L} u_{0}\right\|_{L^{2}(\Omega)} \leq C\left\{(1+t)^{-1 / 4-l / 2}\left\|u_{0}\right\|_{L^{1}\left(\mathbb{R}: L^{2}(D)\right)}+e^{-d t}\left\|u_{0}\right\|_{H^{1}(\Omega)}\right\}
$$

holds for $t \geq 0$ and $0 \leq k+l \leq 1$ with positive constants $C$ and $d$.

To prove Theorem 3.1, we consider the Fourier transform of (3.1) in $x_{3}$ variable which is written as

$$
\begin{gathered}
\partial_{t} \widehat{\phi}+i \xi v_{s}^{3} \widehat{\phi}+\gamma^{2} \nabla^{\prime} \cdot\left(\rho_{s} \widehat{w}^{\prime}\right)+\gamma^{2} i \xi \rho_{s} \widehat{w}^{3}=0, \\
\partial_{t} \widehat{w}^{\prime}-\frac{v}{\rho_{s}}\left(\Delta^{\prime}-\xi^{2}\right) \widehat{w}^{\prime}-\frac{\tilde{v}}{\rho_{s}} \nabla^{\prime}\left(\nabla^{\prime} \cdot \widehat{w}^{\prime}+i \xi \widehat{w}^{3}\right)+\nabla^{\prime}\left(\frac{P^{\prime}\left(\rho_{s}\right)}{\gamma^{2} \rho_{s}} \widehat{\phi}\right)+i \xi v_{s}^{3} \widehat{w}^{\prime}=0, \\
\partial_{t} \widehat{w}^{3}-\frac{v}{\rho_{s}}\left(\Delta^{\prime}-\xi^{2}\right) \widehat{w}^{3}-\frac{\tilde{v}}{\rho_{s}} i \xi\left(\nabla^{\prime} \cdot \widehat{w}^{\prime}+i \xi \widehat{w}^{3}\right)+i \xi\left(\frac{P^{\prime}\left(\rho_{s}\right)}{\gamma^{2} \rho_{s}} \widehat{\phi}\right) \\
+i \xi v_{s}^{3} \widehat{w}^{3}+\frac{v}{\gamma^{2} \rho_{s}^{2}} \Delta^{\prime} v_{s}^{3} \widehat{\phi}+\widehat{w}^{\prime} \cdot \nabla^{\prime} v_{s}^{3}=0, \\
\left.\widehat{w}\right|_{\partial D}=0
\end{gathered}
$$

for $t>0$, and

$$
\left.\mathrm{T}_{(}(\widehat{\phi}, \widehat{w})\right|_{t=0}={ }^{\mathrm{T}}\left(\widehat{\phi}_{0}, \widehat{w}_{0}\right)=\widehat{u}_{0}
$$

We thus arrive at the following problem

$$
\partial_{t} \widehat{u}+\widehat{L} \xi \widehat{u}=0,\left.\quad \widehat{u}\right|_{t=0}=\widehat{u}_{0}
$$

with a parameter $\xi \in \mathbb{R}$. Here $\widehat{u}={ }^{\mathrm{T}}\left(\widehat{\phi}\left(x^{\prime}, t\right), \widehat{w}\left(x^{\prime}, t\right)\right) \in D\left(\widehat{L}_{\xi}\right) \quad\left(x^{\prime} \in D, t>0\right), \widehat{u}_{0} \in$ $H^{1}(D) \times H_{0}^{1}(D)$, and $\widehat{L}_{\xi}$ is the operator on $L^{2}(D)$ of the form

$$
\widehat{L}_{\xi}=\widehat{A}_{\xi}+\widehat{B}_{\xi}+\widehat{C}_{0},
$$


where

$$
\begin{gathered}
\widehat{A}_{\xi}=\left(\begin{array}{ccc}
0 & 0 & 0 \\
0 & -\frac{v}{\rho_{s}}\left(\Delta^{\prime}-|\xi|^{2}\right) I_{2}-\frac{\tilde{v}}{\rho_{s}} \nabla^{\prime} \nabla^{\prime} . & -i \frac{\tilde{v}}{\rho_{s}} \xi \nabla^{\prime} \\
0 & -i \frac{\tilde{v}}{\rho_{s}} \xi \nabla^{\prime} . & -\frac{v}{\rho_{s}}\left(\Delta^{\prime}-|\xi|^{2}\right)+\frac{\tilde{v}}{\rho_{s}}|\xi|^{2}
\end{array}\right), \\
\widehat{B}_{\xi}=\left(\begin{array}{ccc}
i \xi v_{s}^{3} & \gamma^{2} \nabla^{\prime} \cdot\left(\rho_{s} \cdot\right) & i \gamma^{2} \rho_{s} \xi \\
\nabla^{\prime}\left(\frac{P^{\prime}\left(\rho_{s}\right)}{\gamma^{2} \rho_{s}}\right) & i \xi v_{s}^{3} I_{2} & 0 \\
i \xi \frac{P^{\prime}\left(\rho_{s}\right)}{\gamma^{2} \rho_{s}} & 0 & i \xi v_{s}^{3}
\end{array}\right), \quad \widehat{C}_{0}=\left(\begin{array}{ccc}
0 & 0 & 0 \\
0 & 0 & 0 \\
\frac{v}{\gamma^{2} \rho_{s}^{2}} \Delta^{\prime} v_{s}^{3} & \mathrm{~T}\left(\nabla^{\prime} v_{s}^{3}\right) & 0
\end{array}\right)
\end{gathered}
$$

with domain of definition

$$
D\left(\widehat{L}_{\xi}\right)=\left\{\widehat{u}={ }^{\mathrm{T}}(\widehat{\phi}, \widehat{w}) \in L^{2}(D) ; \widehat{w} \in H_{0}^{1}(D), \widehat{L} \xi \widehat{u} \in L^{2}(D)\right\} .
$$

Note that $D\left(\widehat{L}_{\xi}\right)=D\left(\widehat{L}_{0}\right)$ for all $\xi \in \mathbb{R}$, where $\widehat{L}_{0}=\left.\widehat{L}_{\xi}\right|_{\xi=0}$.

As in the case of $L$, following [4], one can show that $-\widehat{L}_{\xi}$ generates a $C_{0}$-semigroup on $L^{2}(D)$. Furthermore if $\widehat{u}_{0} \in H^{1}(D) \times H_{0}^{1}(D)$, then

$$
\begin{aligned}
e^{-t \widehat{L}_{\xi}} \widehat{u}_{0} & \in C\left([0, T] ; H^{1}(D) \times H_{0}^{1}(D)\right), \\
Q_{0} e^{-t \widehat{L}_{\xi}} \widehat{u}_{0} & \in H^{1}\left(0, T ; H^{1}(D)\right), \\
\tilde{Q} e^{-t \widehat{L}_{\xi}} \widehat{u}_{0} & \in L^{2}\left(0, T ; H^{2}(D)\right) \cap H^{1}\left(0, T ; L^{2}(D)\right)
\end{aligned}
$$

for any $T>0$.

To prove Theorem 3.1 we decompose $e^{-t L} u_{0}$ in the following way. We define $\chi_{1}(\xi)$ and $\chi_{\infty}(\xi)$ by $\chi_{1}(\xi)=1$ if $|\xi| \leq 1, \chi_{1}(\xi)=0$ if $|\xi|>1$, and $\chi_{\infty}(\xi)=1-\chi_{1}(\xi)$.

We decompose $e^{-t L} u_{0}$ as

$$
e^{-t L} u_{0}=U_{1}(t) u_{0}+U_{\infty}(t) u_{0}
$$

where

$$
U_{j}(t) u_{0}=\mathcal{F}^{-1}\left[\chi_{j} e^{-t \widehat{L}_{\xi}} \widehat{u}_{0}\right], \quad j=1, \infty .
$$

We can then obtain the following decay estimates for $U_{1}(t) u_{0}$ and $U_{\infty}(t) u_{0}$.

THEOREM 3.2. There exist positive constants $v_{0}, \gamma_{0}, \omega_{0}$ and $d$ such that if $v \geq v_{0}$, $\gamma^{2} /(v+\tilde{v}) \geq \gamma_{0}^{2}$ and $(v+\tilde{v}) \omega / v \leq \omega_{0}$, then for any $l=0,1, \ldots$, there exists a positive constant $C=C(l)$ such that the estimates

$$
\begin{aligned}
\left\|\partial_{x_{3}}^{l} U_{1}(t) u_{0}\right\|_{L^{2}} & \leq C(1+t)^{-1 / 4-l / 2}\left\|u_{0}\right\|_{L^{1}\left(\mathbb{R}: L^{2}(D)\right)}, \\
\left\|\partial_{x^{\prime}} \partial_{x_{3}}^{l} U_{1}(t) u_{0}\right\|_{L^{2}} & \leq C\left\{(1+t)^{-1 / 4-l / 2}\left\|u_{0}\right\|_{L^{1}\left(\mathbb{R}: L^{2}(D)\right)}+e^{-d t}\left(\left\|u_{0}\right\|_{L^{2}}+\left\|\partial_{x^{\prime}} u_{0}\right\|_{L^{2}}\right)\right\}
\end{aligned}
$$

hold for $t \geq 0$. 
THEOREM 3.3. There exist positive constants $v_{0}, \gamma_{0}, \omega_{0}$ and $d$ such that if $v \geq v_{0}$, $\gamma^{2} /(v+\tilde{v}) \geq \gamma_{0}^{2}$ and $\omega \leq \omega_{0}$, then the estimate

$$
\left\|U_{\infty}(t) u_{0}\right\|_{H^{1}} \leq C e^{-d t}\left\|u_{0}\right\|_{H^{1}}
$$

holds for $t \geq 0$ with a positive constant $C$.

Theorem 3.1 follows from Theorems 3.2 and 3.3. In Section 4 we will prove Theorem 3.2 and in Section 5 we will give an outline of the proof of Theorem 3.3.

\section{Decay estimate of the low-frequency part}

In this section we give a proof of Theorem 3.2. Theorem 3.2 is a consequence of Propositions 4.12 and 4.20 below.

For simplicity we omit $\widehat{\cdot}$ of $\widehat{u}, \widehat{\phi}$ and $\widehat{w}$ in (3.3)-(3.8).

To prove Theorem 3.2 we decompose $u(t)$ based on a spectral property of $\widehat{L}_{\xi}$ with $\xi=0$, namely, $\widehat{L}_{0}$.

We introduce the adjoint operator $\widehat{L}_{\xi}^{*}$ of $\widehat{L}_{\xi}$ with the weighted inner product $\langle\cdot, \cdot\rangle$. We define $\widehat{L}_{\xi}^{*}$ by

$$
\widehat{L}_{\xi}^{*}=\widehat{A}_{\xi}^{*}+\widehat{B}_{\xi}^{*}+\widehat{C}_{0}^{*}
$$

with domain of definition

$$
D\left(\widehat{L}_{\xi}^{*}\right)=\left\{u={ }^{\mathrm{T}}(\phi, w) \in L^{2}(D) ; w \in H_{0}^{1}(D), \widehat{L}_{\xi}^{*} u \in L^{2}(D)\right\},
$$

where

$$
\widehat{A}_{\xi}^{*}=\widehat{A}_{\xi}, \quad \widehat{B}_{\xi}^{*}=-\widehat{B}_{\xi}
$$

and

$$
\widehat{C}_{0}^{*}=\left(\begin{array}{ccc}
0 & 0 & \frac{\gamma^{2} v \Delta^{\prime} v_{s}^{3}}{P^{\prime}\left(\rho_{s}\right)} \\
0 & 0 & \nabla^{\prime} v_{s}^{3} \\
0 & 0 & 0
\end{array}\right)
$$

Note that $D\left(\widehat{L}_{\xi}\right)=D\left(\widehat{L}_{\xi}^{*}\right)$ for any $\xi \in \mathbb{R}$. It follows that

$$
\begin{gathered}
\left\langle\widehat{A}_{\xi} u, v\right\rangle=\left\langle u, \widehat{A}_{\xi}^{*} v\right\rangle=\left\langle u, \widehat{A}_{\xi} v\right\rangle, \\
\left\langle\widehat{B}_{\xi} u, v\right\rangle=\left\langle u, \widehat{B}_{\xi}^{*} v\right\rangle=-\left\langle u, \widehat{B}_{\xi} v\right\rangle, \\
\left\langle\widehat{C}_{0} u, v\right\rangle=\left\langle u, \widehat{C}_{0}^{*} v\right\rangle
\end{gathered}
$$

and

$$
\left\langle\widehat{L}_{\xi} u, v\right\rangle=\left\langle u, \widehat{L}_{\xi}^{*} v\right\rangle
$$

for $u, v \in D\left(\widehat{L}_{\xi}\right)$.

We begin with a lemma on the zero-eigenvalue of $\widehat{L}_{0}$ and $\widehat{L}_{0}^{*}$. 
LEMMA 4.1. (i) There exists a constant $\omega_{0}>0$ such that if $(v+\tilde{v}) \omega / v \leq \omega_{0}$, then $\lambda=0$ is a simple eigenvalue of $\widehat{L}_{0}$ and $\widehat{L}_{0}^{*}$.

(ii) The eigenspaces for $\lambda=0$ of $\widehat{L}_{0}$ and $\widehat{L}_{0}^{*}$ are spanned by $u^{(0)}$ and $u^{(0) *}$, respectively, where

$$
u^{(0)}={ }^{\mathrm{T}}\left(\phi^{(0)}, w^{(0)}\right), \quad w^{(0)}={ }^{\mathrm{T}}\left(0,0, w^{(0), 3}\right)
$$

and

$$
u^{(0) *}={ }^{\mathrm{T}}\left(\phi^{(0) *}, 0\right)
$$

Here

$$
\phi^{(0)}\left(x^{\prime}\right)=\alpha_{0} \frac{\gamma^{2} \rho_{s}\left(x^{\prime}\right)}{P^{\prime}\left(\rho_{s}\left(x^{\prime}\right)\right)}, \quad \alpha_{0}=\left(\int_{D} \frac{\gamma^{2} \rho_{s}\left(x^{\prime}\right)}{P^{\prime}\left(\rho_{s}\left(x^{\prime}\right)\right)} d x^{\prime}\right)^{-1} ;
$$

and $w^{(0), 3}$ is the solution of the following problem

$$
\left\{\begin{array}{l}
-\Delta^{\prime} w^{(0), 3}=-\frac{1}{\gamma^{2} \rho_{s}} \Delta^{\prime} v_{s}^{3} \phi^{(0)} \\
\left.w^{(0), 3}\right|_{\partial D}=0
\end{array}\right.
$$

and

$$
\phi^{(0) *}\left(x^{\prime}\right)=\frac{\gamma^{2}}{\alpha_{0}} \phi^{(0)}\left(x^{\prime}\right) .
$$

(iii) The eigenprojections $\widehat{\Pi}^{(0)}$ and $\widehat{\Pi}^{(0) *}$ for $\lambda=0$ of $\widehat{L}_{0}$ and $\widehat{L}_{0}^{*}$ are given by

$$
\begin{gathered}
\widehat{\Pi}^{(0)} u=\left\langle u, u^{(0) *}\right\rangle u^{(0)}=\left\langle Q_{0} u\right\rangle u^{(0)}, \\
\widehat{\Pi}^{(0) *} u=\left\langle u, u^{(0)}\right\rangle u^{(0) *}
\end{gathered}
$$

for $u={ }^{\mathrm{T}}(\phi, w)$, respectively.

(iv) Let $u^{(0)}$ be written as $u^{(0)}=u_{0}^{(0)}+u_{1}^{(0)}$, where

$$
u_{0}^{(0)}={ }^{\mathrm{T}}\left(\phi^{(0)}, 0\right), \quad u_{1}^{(0)}={ }^{\mathrm{T}}\left(0, w^{(0)}\right) .
$$

Then

$$
u^{(0) *}=\frac{\gamma^{2}}{\alpha_{0}} u_{0}^{(0)}
$$

and

$$
\left\langle u, u^{(0)}\right\rangle=\frac{\alpha_{0}}{\gamma^{2}}\langle\phi\rangle+\left(w^{3}, w^{(0), 3} \rho_{s}\right)
$$

for $u={ }^{\mathrm{T}}(\phi, w)={ }^{\mathrm{T}}\left(\phi, w^{\prime}, w^{3}\right)$.

Remark 4.2. We have $\phi^{(0)}=O(1), \alpha_{0}=O(1)$ and $w^{(0), 3}=O\left(1 / \gamma^{2}\right)$ as $\gamma^{2} \rightarrow \infty$.

Proof. Let $\widehat{L}_{0} u=0$ for $u={ }^{\mathrm{T}}\left(\phi, w^{\prime}, w^{3}\right) \in D\left(\widehat{L}_{0}\right)$. Then

$$
\left\{\begin{array}{l}
\gamma^{2} \nabla^{\prime} \cdot\left(\rho_{s} w^{\prime}\right)=0, \\
-\frac{v}{\rho_{s}} \Delta^{\prime} w^{\prime}-\frac{\tilde{v}}{\rho_{s}} \nabla^{\prime} \nabla^{\prime} \cdot w^{\prime}+\nabla^{\prime}\left(\frac{P^{\prime}\left(\rho_{s}\right)}{\gamma^{2} \rho_{s}} \phi\right)=0, \\
-\frac{v}{\rho_{s}} \Delta^{\prime} w^{3}+\frac{v}{\gamma^{2} \rho_{s}^{2}} \Delta^{\prime} v_{s}^{3} \phi+w^{\prime} \cdot \nabla^{\prime} v_{s}^{3}=0, \\
\left.w\right|_{\partial D}=0 .
\end{array}\right.
$$


We take the weighted inner product of $(4.1)$ with $^{\mathrm{T}}\left(\phi, w^{\prime}, 0\right)$ to get

$$
v\left|\nabla^{\prime} w^{\prime}\right|_{2}^{2}+\tilde{v}\left|\nabla^{\prime} \cdot w^{\prime}\right|_{2}^{2}=0 .
$$

Since $w^{\prime} \in H_{0}^{1}(D)$, we have $w^{\prime}=0$. It then follows that

$$
\left\{\begin{array}{l}
\nabla^{\prime}\left(\frac{P^{\prime}\left(\rho_{s}\right)}{\gamma^{2} \rho_{s}} \phi\right)=0 \\
-\Delta^{\prime} w^{3}=-\frac{1}{\gamma^{2} \rho_{s}} \Delta^{\prime} v_{s}^{3} \phi \\
\left.w^{3}\right|_{\partial D}=0
\end{array}\right.
$$

This implies that $\left(P^{\prime}\left(\rho_{S}\right) / \gamma^{2} \rho_{s}\right) \phi$ is a constant since $D$ is connected, and we conclude that $\operatorname{Ker}\left(\widehat{L}_{0}\right)=\operatorname{span}\left\{u^{(0)}\right\}$. Note that $\int_{D} \phi^{(0)} d x^{\prime}=1$.

Let $\widehat{L}_{0}^{*} u=0$ for $u={ }^{\mathrm{T}}\left(\phi, w^{\prime}, w^{3}\right)$. Then

$$
\left\{\begin{array}{l}
-\gamma^{2} \nabla^{\prime} \cdot\left(\rho_{s} w^{\prime}\right)+\frac{\gamma^{2} v}{P^{\prime}\left(\rho_{s}\right)} \Delta^{\prime} v_{s}^{3} w^{3}=0, \\
-\frac{v}{\rho_{s}} \Delta^{\prime} w^{\prime}-\frac{\tilde{v}}{\rho_{s}} \nabla^{\prime} \nabla^{\prime} \cdot w^{\prime}-\nabla^{\prime}\left(\frac{P^{\prime}\left(\rho_{s}\right)}{\gamma^{2} \rho_{s}} \phi\right)+w^{3} \nabla^{\prime} v_{s}^{3}=0 \\
-\frac{v}{\rho_{s}} \Delta^{\prime} w^{3}=0 \\
\left.w\right|_{\partial D}=0 .
\end{array}\right.
$$

The third equation, together with $\left.w^{3}\right|_{\partial D}=0$, implies that $w^{3}=0$ and, hence,

$$
\left\{\begin{array}{l}
-\gamma^{2} \nabla^{\prime} \cdot\left(\rho_{s} w^{\prime}\right)=0, \\
-\frac{v}{\rho_{s}} \Delta^{\prime} w^{\prime}-\frac{\tilde{v}}{\rho_{s}} \nabla^{\prime} \nabla^{\prime} \cdot w^{\prime}-\nabla^{\prime}\left(\frac{P^{\prime}\left(\rho_{s}\right)}{\gamma^{2} \rho_{s}} \phi\right)=0 \\
\left.w^{\prime}\right|_{\partial D}=0 .
\end{array}\right.
$$

Similarly to the case of $\widehat{L}_{0}$, one can show that $w^{\prime}=0$ and $\left(P^{\prime}\left(\rho_{s}\right) / \gamma^{2} \rho_{s}\right) \phi$ is a constant. We set $\phi^{(0) *}=\left(\gamma^{2} / \alpha_{0}\right) \phi^{(0)}\left(x^{\prime}\right)$. Note that $\int_{D} \phi^{(0)} \overline{\phi^{(0) *}}\left(P^{\prime}\left(\rho_{s}\right) / \gamma^{4} \rho_{s}\right) d x^{\prime}=1$. We thus proved (i), (ii) and (iii) except for the simplicity of $\lambda=0$. The assertion (iv) can be verified by direct computations.

It remains to prove the simplicity of $\lambda=0$. Since we have already proved that $\operatorname{Ker}\left(\widehat{L}_{0}\right)=$ $\operatorname{span}\left\{u^{(0)}\right\}$ and $\operatorname{Ker}\left(\widehat{L}_{0}^{*}\right)=\operatorname{span}\left\{u^{(0) *}\right\}$, if we would prove the following lemma, then the proof of the simplicity of $\lambda=0$ would be complete.

LEMMA 4.3. There exists a constant $\omega_{0}>0$ such that if $(v+\tilde{v}) \omega / v \leq \omega_{0}$, then $R\left(\widehat{L}_{0}\right)$ and $R\left(\widehat{L}_{0}^{*}\right)$ are closed, and it holds that

$$
L^{2}(D)=\operatorname{Ker}\left(\widehat{L}_{0}\right) \oplus R\left(\widehat{L}_{0}\right), \quad L^{2}(D)=\operatorname{Ker}\left(\widehat{L}_{0}^{*}\right) \oplus R\left(\widehat{L}_{0}^{*}\right) .
$$

To prove Lemma 4.3, we show the following proposition. 
Proposition 4.4. There exists a constant $\omega_{0}>0$ such that if $(v+\tilde{v}) \omega / v \leq \omega_{0}$, then for any $f={ }^{\mathrm{T}}\left(f^{0}, g\right)={ }^{\mathrm{T}}\left(f^{0}, g^{\prime}, g^{3}\right) \in L^{2}(D)$ with $\left\langle f^{0}\right\rangle=0$, there is a unique solution $u=$ $\mathrm{T}_{(}(\phi, w) \in D\left(\widehat{L}_{0}\right)$ of the following problem:

$$
\left\{\begin{array}{l}
\widehat{L}_{0} u=f, \\
\langle\phi\rangle=0 .
\end{array}\right.
$$

Proof. Let us prove that if $\left\langle f^{0}\right\rangle=0$, then (4.2) has a unique solution $u={ }^{\mathrm{T}}(\phi, w) \in L^{2}(D) \times$ $H_{0}^{1}(D)$ with $\langle\phi\rangle=0$. The problem (4.2) is rewritten as the following system:

$$
\left\{\begin{array}{l}
\nabla^{\prime} \cdot w^{\prime}=F\left[w^{\prime} ; f^{0}\right], \\
-v \Delta^{\prime} w^{\prime}+\nabla^{\prime} \phi=G^{\prime}\left[\phi, w^{\prime} ; f^{0}, g^{\prime}\right] \\
\left.w^{\prime}\right|_{\partial D}=0 \\
\langle\phi\rangle=0
\end{array}\right.
$$

and

$$
\left\{\begin{array}{l}
-v \Delta^{\prime} w^{3}=G^{3}\left[\phi, w^{\prime} ; g^{3}\right] \\
\left.w^{3}\right|_{\partial D}=0
\end{array}\right.
$$

where

$$
\begin{gathered}
F\left[w^{\prime} ; f^{0}\right]=\nabla^{\prime} \cdot\left(\left(1-\rho_{s}\right) w^{\prime}\right)+\frac{1}{\gamma^{2}} f^{0}, \\
G^{\prime}\left[\phi, w^{\prime} ; f^{0}, g^{\prime}\right]= \\
\tilde{v} \nabla^{\prime} F\left[w^{\prime} ; f^{0}\right]+\nabla^{\prime}\left(\left(1-\rho_{s}\right) \phi\right)+\nabla^{\prime} \rho_{s} \phi \\
+\rho_{s} \nabla^{\prime}\left(\left(1-\frac{P^{\prime}\left(\rho_{s}\right)}{\gamma^{2} \rho_{s}}\right) \phi\right)+\rho_{s} g^{\prime}, \\
G^{3}\left[\phi, w^{\prime} ; g^{3}\right]=-\frac{v}{\gamma^{2} \rho_{s}} \Delta^{\prime} v_{s}^{3} \phi-\rho_{s} w^{\prime} \cdot \nabla^{\prime} v_{s}^{3}+\rho_{s} g^{3} .
\end{gathered}
$$

We define a set $\dot{X}$ by

$$
\dot{X}=\left\{\left(p, v^{\prime}\right) ; p \in L^{2}(D), v^{\prime}={ }^{\mathrm{T}}\left(v^{1}, v^{2}\right) \in H_{0}^{1}(D),\langle p\rangle=0\right\}
$$

with norm

$$
\left|\left(p, v^{\prime}\right)\right|_{\dot{X}}=|p|_{2}+v\left|\nabla^{\prime} v^{\prime}\right|_{2}
$$

We assume that $\left(\tilde{\phi}, \tilde{w}^{\prime}\right) \in \dot{X}$. Let us consider the following problem

$$
\left\{\begin{array}{l}
\nabla^{\prime} \cdot w^{\prime}=F\left[\tilde{w}^{\prime} ; f^{0}\right], \\
-v \Delta^{\prime} w^{\prime}+\nabla^{\prime} \phi=G^{\prime}\left[\tilde{\phi}, \tilde{w}^{\prime} ; f^{0}, g^{\prime}\right], \\
\left.w^{\prime}\right|_{\partial D}=0 .
\end{array}\right.
$$

It holds that

$$
\begin{gathered}
F\left[\tilde{w}^{\prime} ; f^{0}\right] \in L^{2}(D), \quad\left\langle F\left[\tilde{w}^{\prime} ; f^{0}\right]\right\rangle=0, \\
G^{\prime}\left[\tilde{\phi}, \tilde{w}^{\prime} ; f^{0}, g^{\prime}\right] \in H^{-1}(D),
\end{gathered}
$$


where $H^{-1}(D)$ denotes the dual space to $H_{0}^{1}(D)$ with norm $|\cdot|_{H^{-1}}$. In fact, we have from the Poincaré inequality that

$$
\begin{gathered}
\left|F\left[\tilde{w}^{\prime} ; f^{0}\right]\right|_{2} \leq\left|\nabla^{\prime} \cdot\left(\left(1-\rho_{S}\right) \tilde{w}^{\prime}\right)\right|_{2}+\frac{1}{\gamma^{2}}\left|f^{0}\right|_{2} \\
\leq C\left\{\omega\left|\tilde{w}^{\prime}\right|_{H^{1}}+\frac{1}{\gamma^{2}}\left|f^{0}\right|_{2}\right\} \\
\leq C\left\{\omega\left|\nabla^{\prime} \tilde{w}^{\prime}\right|_{2}+\frac{1}{\gamma^{2}}\left|f^{0}\right|_{2}\right\} \\
\left|G^{\prime}\left[\tilde{\phi}, \tilde{w}^{\prime} ; f^{0}, g^{\prime}\right]\right|_{H^{-1}} \leq \\
C\left\{\left|\nabla^{\prime} F\left[\tilde{w}^{\prime} ; f^{0}\right]\right|_{H^{-1}}+\left|\nabla^{\prime}\left(\left(1-\rho_{s}\right) \tilde{\phi}\right)\right|_{H^{-1}}\right. \\
\left.+\left|\nabla^{\prime} \rho_{s} \tilde{\phi}\right|_{H^{-1}}+\left|\rho_{S} \nabla^{\prime}\left(\left(1-\frac{P^{\prime}\left(\rho_{S}\right)}{\gamma^{2} \rho_{s}}\right) \tilde{\phi}\right)\right|_{H^{-1}}+\left|g^{\prime}\right|_{H^{-1}}\right\} \\
\leq C\left\{\tilde{v}\left|F\left[\tilde{w}^{\prime} ; f^{0}\right]\right|_{2}+\omega|\tilde{\phi}|_{2}+\left|g^{\prime}\right|_{2}\right\} \\
\leq C\left\{\omega\left(|\tilde{\phi}|_{2}+\tilde{v}\left|\nabla^{\prime} \tilde{w}^{\prime}\right|_{2}\right)+\frac{\tilde{v}}{\gamma^{2}}\left|f^{0}\right|_{2}+\left|g^{\prime}\right|_{2}\right\} .
\end{gathered}
$$

From [19, III.1.4, Theorem 1.4.1], we see that there is a unique solution $\left(\phi, w^{\prime}\right) \in \dot{X}$ of (4.5) and the following estimate holds:

$$
\begin{aligned}
|\phi|_{2}+v\left|\nabla^{\prime} w^{\prime}\right|_{2} & \leq C\left\{\left.v\left|F\left[\tilde{w}^{\prime} ; f^{0}\right]\right|\right|_{2}+\left|G^{\prime}\left[\tilde{\phi}, \tilde{w}^{\prime} ; f^{0}, g^{\prime}\right]\right|_{H^{-1}}\right\} \\
& \leq C_{1}\left\{\omega\left(|\tilde{\phi}|_{2}+(\nu+\tilde{v})\left|\nabla^{\prime} \tilde{w}^{\prime}\right|_{2}\right)+\frac{\nu+\tilde{v}}{\gamma^{2}}\left|f^{0}\right|_{2}+\left|g^{\prime}\right|_{2}\right\},
\end{aligned}
$$

where $C_{1}$ is a positive constant. Let us define a map $\Gamma: \dot{X} \rightarrow \dot{X}$ by

$$
\Gamma\left(\tilde{\phi}, \tilde{w}^{\prime}\right)=\left(\phi, w^{\prime}\right) \quad \text { for }\left(\tilde{\phi}, \tilde{w}^{\prime}\right) \in \dot{X}
$$

where $\left(\phi, w^{\prime}\right) \in \dot{X}$ is a solution of (4.5). We see from (4.6) that

$$
\left|\Gamma\left(\tilde{\phi}, \tilde{w}^{\prime}\right)\right|_{\dot{X}} \leq C_{1}\left\{\omega\left(|\tilde{\phi}|_{2}+(v+\tilde{v})\left|\nabla^{\prime} \tilde{w}^{\prime}\right|_{2}\right)+\frac{v+\tilde{v}}{\gamma^{2}}\left|f^{0}\right|_{2}+\left|g^{\prime}\right|_{2}\right\} .
$$

Since we have the estimate

$$
\left|\Gamma\left(\tilde{\phi}_{1}, \tilde{w}_{1}^{\prime}\right)-\Gamma\left(\tilde{\phi}_{2}, \tilde{w}_{2}^{\prime}\right)\right|_{\dot{X}} \leq C_{1} \omega\left\{|\tilde{\phi}|_{2}+(v+\tilde{v})\left|\nabla^{\prime} \tilde{w}^{\prime}\right|_{2}\right\}
$$

for $\left(\tilde{\phi}_{1}, \tilde{w}_{1}^{\prime}\right),\left(\tilde{\phi}_{2}, \tilde{w}_{2}^{\prime}\right) \in \dot{X}$, if we take $\omega$ sufficiently small satisfying $\omega<\left(1 / 2 C_{1}\right) \min \{1$, $v /(v+\tilde{v})\}$, then we see that $\Gamma: \dot{X} \rightarrow \dot{X}$ is a contraction map. This implies that there is a unique $\left(\phi, w^{\prime}\right) \in \dot{X}$ such that $\Gamma\left(\phi, w^{\prime}\right)=\left(\phi, w^{\prime}\right)$, i.e. there is a unique solution $\left(\phi, w^{\prime}\right) \in \dot{X}$ of (4.3).

Furthermore, for a solution $\left(\phi, w^{\prime}\right) \in \dot{X}$ of (4.3), since

$$
G^{3}\left[\phi, w^{\prime} ; g^{3}\right] \in L^{2}(D),
$$

there is a unique solution $w^{3} \in H_{0}^{1}(D)$ of (4.4). Consequently, we have

$$
\widehat{L}_{0} u=f \quad \text { in the sense of distribution, }
$$


where $f={ }^{\mathrm{T}}\left(f^{0}, g^{\prime}, g^{3}\right) \in L^{2}(D)$ with $\left\langle f^{0}\right\rangle=0$. Since $f \in L^{2}(D)$, it holds that $\widehat{L}_{0} u \in$ $L^{2}(D)$. It then follows that

$$
u \in D\left(\widehat{L}_{0}\right) .
$$

This completes the proof.

Proof of Lemma 4.3. We have already proved that

$$
\operatorname{Ker}\left(\widehat{L}_{0}\right)=\widehat{\Pi}^{(0)} L^{2}(D) .
$$

To prove $R\left(\widehat{L}_{0}\right)=\left(I-\widehat{\Pi}^{(0)}\right) L^{2}(D)$, we first show that

$$
u={ }^{\mathrm{T}}(\phi, w) \in\left(I-\widehat{\Pi}^{(0)}\right) L^{2}(D) \quad \text { if and only if }\langle\phi\rangle=0 .
$$

Let us prove (4.7). We can decompose $u={ }^{\mathrm{T}}(\phi, w)$ as

$$
u=\langle\phi\rangle u^{(0)}+u_{1}
$$

Here

$$
\langle\phi\rangle u^{(0)} \in \Pi^{(0)} L^{2}(D), \quad u_{1}={ }^{\mathrm{T}}\left(\phi_{1}, w_{1}\right) \in\left(I-\Pi^{(0)}\right) L^{2}(D) .
$$

This implies that if $\langle\phi\rangle=0$, then

$$
u=\langle\phi\rangle u^{(0)}+u_{1}=u_{1} \in\left(I-\widehat{\Pi}^{(0)}\right) L^{2}(D) .
$$

On the other hand, if $u={ }^{\mathrm{T}}(\phi, w) \in\left(I-\widehat{\Pi}^{(0)}\right) L^{2}(D)$, then there exists $\tilde{u}={ }^{\mathrm{T}}(\tilde{\phi}, \tilde{w}) \in L^{2}(D)$ such that

$$
u=\tilde{u}-\langle\tilde{\phi}\rangle u^{(0)} .
$$

It then follows that

$$
\langle\phi\rangle=\langle\tilde{\phi}\rangle-\langle\tilde{\phi}\rangle=0 .
$$

We thus conclude that (4.7) holds true.

We next show that $R\left(\widehat{L}_{0}\right)=\left(I-\widehat{\Pi}^{(0)}\right) L^{2}(D)$. Since $\left\langle Q_{0} \widehat{L}_{0} u\right\rangle=\left\langle\nabla^{\prime} \cdot\left(\rho_{s} w^{\prime}\right)\right\rangle=0$, we see from (4.7) that $\widehat{L}_{0} u \in\left(I-\widehat{\Pi}^{(0)}\right) L^{2}(D)$ and, therefore,

$$
R\left(\widehat{L}_{0}\right) \subset\left(I-\Pi^{(0)}\right) L^{2}(D) .
$$

On the other hand, if $f={ }^{\mathrm{T}}\left(f^{0}, g^{\prime}, g^{3}\right) \in\left(I-\widehat{\Pi}^{(0)}\right) L^{2}(D)$, then it follows from (4.7) that $\left\langle f^{0}\right\rangle=0$. By Proposition 4.4, there exists a unique solution $u={ }^{\mathrm{T}}(\phi, w) \in D\left(\widehat{L}_{0}\right)$ such that $\widehat{L}_{0} u=f$ with $\langle\phi\rangle=0$. This implies that $f \in R\left(\widehat{L}_{0}\right)$ and, thus,

$$
\left(I-\Pi^{(0)}\right) L^{2}(D) \subset R\left(\widehat{L}_{0}\right) .
$$

Therefore, we see that $R\left(\widehat{L}_{0}\right)=\left(I-\widehat{\Pi}^{(0)}\right) L^{2}(D)$. Consequently, we have that $R\left(\widehat{L}_{0}\right)$ is closed and

$$
L^{2}(D)=\operatorname{Ker}\left(\widehat{L}_{0}\right) \oplus R\left(\widehat{L}_{0}\right) .
$$

Similarly, one can prove that $\operatorname{Ker}\left(\widehat{L}_{0}^{*}\right)=\widehat{\Pi}^{(0) *} L^{2}(D)$ and $R\left(\widehat{L}_{0}^{*}\right)=\left(I-\widehat{\Pi}^{(0) *}\right) L^{2}(D)$. We thus see that $R\left(\widehat{L}_{0}^{*}\right)$ is closed and

$$
L^{2}(D)=\operatorname{Ker}\left(\widehat{L}_{0}^{*}\right) \oplus R\left(\widehat{L}_{0}^{*}\right) .
$$

This completes the proof of Lemma 4.1. 
We are now ready to prove Theorem 3.2. We decompose $u(t)$ as follows

$$
\begin{aligned}
u(t) & =\sigma(t) u^{(0)}+u_{1}(t), \\
\sigma(t) & =\left\langle Q_{0} u(t)\right\rangle=\left\langle u(t), u^{(0) *}\right\rangle, \\
u_{1}(t) & =\left(I-\widehat{\Pi}^{(0)}\right) u(t) .
\end{aligned}
$$

The density component of $u_{1}$ is denoted by $\phi_{1}$ and the velocity component is denoted by $w_{1}$, namely,

$$
u_{1}={ }^{\mathrm{T}}\left(\phi_{1}, w_{1}\right) \text {. }
$$

Note that $\left\langle\phi_{1}\right\rangle=0$ and $\left.w_{1}\right|_{\partial D}=0$; the latter follows from $u^{(0)} \in D\left(\widehat{L}_{0}\right)$ which implies that $\left.w^{(0), 3}\right|_{\partial D}=0$.

Remark 4.5. (i) The boundary condition $\left.w_{1}\right|_{\partial D}=0$ implies that the Poincaré inequality holds for $w_{1}:\left|w_{1}\right|_{2} \leq C\left|\partial_{x^{\prime}} w_{1}\right|_{2}$.

(ii) The vanishing mean value condition $\left\langle\phi_{1}\right\rangle=0$ implies that the Poincaré inequality holds for $\phi_{1}:\left|\phi_{1}\right|_{2} \leq C\left|\partial_{x^{\prime}} \phi_{1}\right|_{2}$.

We define $\tilde{M}_{\xi}$ by

$$
\tilde{M}_{\xi}=\widehat{L}_{\xi}-\widehat{L}_{0}=\tilde{A}_{\xi}+\tilde{B}_{\xi},
$$

where

$$
\begin{gathered}
\tilde{A}_{\xi}=\widehat{A}_{\xi}-\widehat{A}_{0}=\left(\begin{array}{ccc}
0 & 0 & 0 \\
0 & \frac{v}{\rho_{s}} \xi^{2} I_{2} & -\frac{\tilde{v}}{\rho_{s}} i \xi \nabla^{\prime} \\
0 & -\frac{\tilde{v}}{\rho_{s}} i \xi \nabla^{\prime} \cdot & \frac{v+\tilde{v}}{\rho_{s}} \xi^{2}
\end{array}\right), \\
\tilde{B}_{\xi}=\widehat{B}_{\xi}-\widehat{B}_{0}=\left(\begin{array}{ccc}
i \xi v_{s}^{3} & 0 & \gamma^{2} i \xi \rho_{s} \\
0 & i \xi v_{s}^{3} I_{2} & 0 \\
i \xi \frac{P^{\prime}\left(\rho_{s}\right)}{\gamma^{2} \rho_{s}} & 0 & i \xi v_{s}^{3}
\end{array}\right) .
\end{gathered}
$$

Decomposing $u(t)$ in (3.8) as $u(t)=\sigma(t) u^{(0)}+u_{1}(t)$, we obtain

$$
\partial_{t}\left(\sigma u^{(0)}+u_{1}\right)+\widehat{L}_{0} u_{1}+\tilde{M}_{\xi}\left(\sigma u^{(0)}+u_{1}\right)=0 .
$$

Applying $\Pi^{(0)}$ and $I-\Pi^{(0)}$ to this equation, we have

$$
\left\{\begin{array}{l}
\partial_{t} \sigma+\left\langle Q_{0} \tilde{M}_{\xi}\left(\sigma u^{(0)}+u_{1}\right)\right\rangle=0, \\
\partial_{t} u_{1}+\widehat{L}_{0} u_{1}+\left(I-\widehat{\Pi}^{(0)}\right) \tilde{M}_{\xi}\left(\sigma u^{(0)}+u_{1}\right)=0 .
\end{array}\right.
$$

Since $\widehat{\Pi}^{(0)} \tilde{M}_{\xi} u=\left\langle Q_{0} \tilde{M}_{\xi} u\right\rangle u^{(0)}$ and $Q_{0} \tilde{M}_{\xi}=Q_{0} \tilde{B}_{\xi}$, we obtain

$$
\begin{aligned}
& \partial_{t} \sigma+\left\langle Q_{0} \tilde{B}_{\xi}\left(\sigma u^{(0)}+u_{1}\right)\right\rangle=0, \\
& \partial_{t} u_{1}+\widehat{L}_{\xi} u_{1}+\tilde{M}_{\xi}\left(\sigma u^{(0)}\right)-\left\langle Q_{0} \tilde{B}_{\xi}\left(\sigma u^{(0)}+u_{1}\right)\right\rangle u^{(0)}=0, \\
& \left.w_{1}\right|_{\partial D}=0, \quad \sigma(0)=\sigma_{0}, \quad u_{1}(0)=u_{1,0},
\end{aligned}
$$


where $\sigma_{0}$ and $u_{1,0}$ are given by

$$
\sigma_{0}=\left\langle u_{0}, u^{(0) *}\right\rangle, \quad u_{1,0}=\left(I-\widehat{\Pi}^{(0)}\right) u_{0} .
$$

We see from (3.9) that if $u_{0} \in H^{1}(D) \times H_{0}^{1}(D)$, then

$$
\begin{aligned}
\sigma & \in H^{1}(0, T), \\
u_{1} & \in C\left([0, T] ; H^{1}(D) \times H_{0}^{1}(D)\right), \\
\phi_{1} & \in H^{1}\left(0, T ; H^{1}(D)\right), \\
w_{1} & \in L^{2}\left(0, T ; H^{2}(D)\right) \cap H^{1}\left(0, T ; L^{2}(D)\right)
\end{aligned}
$$

for all $T>0$.

LEMMA 4.6. For $u_{1}={ }^{\mathrm{T}}\left(\phi_{1}, w_{1}^{\prime}, w_{1}^{3}\right) \in R\left(I-\widehat{\Pi}^{(0)}\right)$, the following estimates hold:

(i) $\left|\left\langle Q_{0} \tilde{B}_{\xi} u^{(0)}\right\rangle\right| \leq C|\xi|$;

(ii) $\left|\left\langle Q_{0} \tilde{B}_{\xi} u_{1}\right\rangle\right| \leq C|\xi|\left(\left|\phi_{1}\right|_{2}+\gamma^{2}\left|w_{1}^{3}\right|_{2}\right)$;

(iii) $\left|\left\langle Q_{0} \tilde{B}_{\xi} u_{1}\right\rangle\right| \leq C\left(|\xi|\left|\phi_{1}\right|_{2}+\gamma^{2}\left|\nabla^{\prime} \cdot w_{1}^{\prime}+i \xi w_{1}^{3}\right|_{2}+\gamma^{2} \omega\left|w_{1}^{\prime}\right|_{2}\right)$.

Lemma 4.6 can be proved by direct computations. We omit the proof.

We will employ an energy method to obtain the decay estimate on solutions of (4.8)(4.10). We write (4.9) as

$$
\left\{\begin{aligned}
\partial_{t} \phi_{1} & +i \xi v_{s}^{3} \phi_{1}+\gamma^{2} \nabla^{\prime} \cdot\left(\rho_{s} w_{1}^{\prime}\right)+\gamma^{2} i \xi \rho_{s} w_{1}^{3} \\
& +i \xi v_{s}^{3} \sigma \phi^{(0)}+\gamma^{2} i \xi \rho_{s} \sigma w^{(0), 3}-\left\langle Q_{0} \tilde{B}_{\xi}\left(\sigma u^{(0)}+u_{1}\right)\right\rangle \phi^{(0)}=0, \\
\partial_{t} w_{1}^{\prime} & -\frac{v}{\rho_{s}}\left(\Delta^{\prime}-\xi^{2}\right) w_{1}^{\prime}-\frac{\tilde{v}}{\rho_{s}} \nabla^{\prime}\left(\nabla^{\prime} \cdot w_{1}^{\prime}+i \xi w_{1}^{3}\right)+\nabla^{\prime}\left(\frac{P^{\prime}\left(\rho_{s}\right)}{\gamma^{2} \rho_{s}} \phi_{1}\right)+i \xi v_{s}^{3} w_{1}^{\prime} \\
& -\frac{\tilde{v}}{\rho_{s}} i \xi \nabla^{\prime}\left(\sigma w^{(0), 3}\right)=0, \\
\partial_{t} w_{1}^{3}- & \frac{v}{\rho_{s}}\left(\Delta^{\prime}-\xi^{2}\right) w_{1}^{3}-\frac{\tilde{v}}{\rho_{s}} i \xi\left(\nabla^{\prime} \cdot w_{1}^{\prime}+i \xi w_{1}^{3}\right)+i \xi\left(\frac{P^{\prime}\left(\rho_{s}\right)}{\gamma^{2} \rho_{s}} \phi_{1}\right)+i \xi v_{s}^{3} w_{1}^{3} \\
& +\frac{v}{\gamma^{2} \rho_{s}^{2}} \Delta^{\prime} v_{s}^{3} \phi_{1}+w_{1}^{\prime} \cdot \nabla^{\prime} v_{s}^{3}+\frac{v+\tilde{v}}{\rho_{s}} \xi^{2} \sigma w^{(0), 3}+i \xi \alpha_{0} \sigma \\
& +i \xi v_{s}^{3} \sigma w^{(0), 3}-\left\langle Q_{0} \tilde{B}_{\xi}\left(\sigma u^{(0)}+u_{1}\right)\right\rangle w^{(0), 3}=0 .
\end{aligned}\right.
$$

Before proceeding further we introduce some notation. For $u={ }^{\mathrm{T}}(\phi, w)$ we define $E_{0}[u]$ by

$$
E_{0}[u]=\frac{1}{\gamma^{2}}\left|\sqrt{\frac{P^{\prime}\left(\rho_{s}\right)}{\gamma^{2} \rho_{s}}} \phi\right|_{2}^{2}+\left|\sqrt{\rho_{s}} w\right|_{2}^{2} .
$$

For $w={ }^{\mathrm{T}}\left(w^{\prime}, w^{3}\right)$ with $w^{\prime}={ }^{\mathrm{T}}\left(w^{1}, w^{2}\right)$ we define $\tilde{D}_{\xi}[w]$ by

$$
\tilde{D}_{\xi}[w]=v\left(\left|\nabla^{\prime} w\right|_{2}^{2}+|\xi|^{2}|w|_{2}^{2}\right)+\tilde{v}\left|\nabla^{\prime} \cdot w^{\prime}+i \xi w^{3}\right|_{2}^{2} .
$$

For $\phi$ we define $\dot{\phi}$ by

$$
\dot{\phi}=\partial_{t} \phi+i \xi v_{s}^{3} \phi
$$


PROPOSITION 4.7. There exist constants $v_{0}>0$ and $\omega_{0}>0$ such that if $v \geq v_{0}$ and $(v+\tilde{v}) \omega / v \leq \omega_{0}$, then the following estimates hold:

$$
\begin{gathered}
\frac{1}{2} \frac{d}{d t}\left(\frac{\alpha_{0}}{\gamma^{2}}|\sigma|^{2}+E_{0}\left[u_{1}\right]\right)+\frac{1}{2} \tilde{D}_{\xi}\left[w_{1}\right] \leq C\left\{\left(\frac{1}{\gamma^{2}}+\frac{v+\tilde{v}}{\gamma^{4}}\right)|\xi|^{2}|\sigma|^{2}+\left(\frac{1}{\gamma^{2}}+\frac{v}{\gamma^{4}}\right)\left|\phi_{1}\right|_{2}^{2}\right\}, \\
\frac{v+\tilde{v}}{\gamma^{4}}\left|\dot{\phi}_{1}\right|_{2}^{2} \leq C\left\{\frac{v+\tilde{v}}{\gamma^{4}}|\xi|^{2}|\sigma|^{2}+\frac{v+\tilde{v}}{\gamma^{4}}|\xi|^{2}\left|\phi_{1}\right|_{2}^{2}+\left(1+\frac{v+\tilde{v}}{v} \omega^{2}\right) \tilde{D}_{\xi}\left[w_{1}\right]\right\} .
\end{gathered}
$$

Proof. Multiplying (4.8) by $\bar{\sigma}(t)$ and taking real part of the resulting equation, we have

$$
\frac{1}{2} \frac{d}{d t}|\sigma|^{2}+\operatorname{Re}\left\{\left\langle Q_{0} \tilde{B}_{\xi}\left(\sigma u^{(0)}+u_{1}\right)\right\rangle \bar{\sigma}\right\}=0 .
$$

Since $\tilde{B}_{\xi}^{*}=-\tilde{B}_{\xi}$ and $u^{(0) *}=\left(\gamma^{2} / \alpha_{0}\right) u_{0}^{(0)}$, we see that

$$
\begin{aligned}
\left\langle Q_{0} \tilde{B}_{\xi} u_{1}\right\rangle \bar{\sigma} & =\left\langle\tilde{B}_{\xi} u_{1}, \sigma u^{(0) *}\right\rangle \\
& =-\left\langle u_{1}, \tilde{B}_{\xi}\left(\sigma u^{(0) *}\right)\right\rangle \\
& =-\frac{\gamma^{2}}{\alpha_{0}}\left\langle u_{1}, \tilde{B}_{\xi}\left(\sigma u_{0}^{(0)}\right)\right\rangle,
\end{aligned}
$$

where $u_{0}^{(0)}={ }^{\mathrm{T}}\left(\phi^{(0)}, 0\right)$. On the other hand, since

$$
\left\langle Q_{0} \tilde{B}_{\xi}\left(\sigma u^{(0)}\right)\right\rangle \bar{\sigma}=i \xi|\sigma|^{2}\left\{\left\langle v_{s}^{3} \phi_{1}\right\rangle+\left\langle\gamma^{2} \rho_{s} w_{1}^{3}\right\rangle\right\}
$$

we have

$$
\operatorname{Re}\left\{\left\langle Q_{0} \tilde{B}_{\xi}\left(\sigma u^{(0)}\right)\right\rangle \bar{\sigma}\right\}=0 .
$$

We thus obtain from (4.14)-(4.16) that

$$
\frac{1}{2} \frac{d}{d t}|\sigma|^{2}-\frac{\gamma^{2}}{\alpha_{0}} \operatorname{Re}\left\langle u_{1}, \tilde{B}_{\xi}\left(\sigma u_{0}^{(0)}\right)\right\rangle=0 .
$$

We next take the weighted inner product of (4.11) with $u_{1}$. The real part of the resulting equation then gives

$$
\begin{aligned}
& \frac{1}{2} \frac{d}{d t} E_{0}\left[u_{1}\right]+\operatorname{Re}\left\langle\widehat{L}_{0} u_{1}, u_{1}\right\rangle+\operatorname{Re}\left\langle\tilde{M}_{\xi}\left(\sigma u^{(0)}+u_{1}\right), u_{1}\right\rangle \\
& -\operatorname{Re}\left\{\left\langle Q_{0} \tilde{B}_{\xi}\left(\sigma u^{(0)}+u_{1}\right)\right\rangle\left\langle u^{(0)}, u_{1}\right\rangle\right\}=0 .
\end{aligned}
$$

Since $\widehat{B}_{\xi}^{*}=-\widehat{B}_{\xi}$, we see that $\operatorname{Re}\left\langle\widehat{B}_{\xi} u_{1}, u_{1}\right\rangle=0$. It then follows that

$$
\begin{aligned}
\operatorname{Re} & \left\langle\widehat{L}_{0} u_{1}, u_{1}\right\rangle+\operatorname{Re}\left\langle\tilde{M}_{\xi}\left(\sigma u^{(0)}+u_{1}\right), u_{1}\right\rangle \\
& =\operatorname{Re}\left\langle\widehat{C}_{0} u_{1}, u_{1}\right\rangle+\operatorname{Re}\left\langle\widehat{A}_{\xi} u_{1}, u_{1}\right\rangle+\operatorname{Re}\left\langle\tilde{A}_{\xi}\left(\sigma u^{(0)}\right), u_{1}\right\rangle+\operatorname{Re}\left\langle\tilde{B}_{\xi}\left(\sigma u^{(0)}\right), u_{1}\right\rangle \\
& =\operatorname{Re}\left\langle\widehat{C}_{0} u_{1}, u_{1}\right\rangle+\tilde{D}_{\xi}\left[w_{1}\right]+\operatorname{Re}\left\langle\tilde{A}_{\xi}\left(\sigma u^{(0)}\right), u_{1}\right\rangle+\operatorname{Re}\left\langle\tilde{B}_{\xi}\left(\sigma u^{(0)}\right), u_{1}\right\rangle .
\end{aligned}
$$

This, together with (4.18), gives

$$
\begin{aligned}
& \frac{1}{2} \frac{d}{d t} E_{0}\left[u_{1}\right]+\tilde{D}_{\xi}\left[w_{1}\right]+\operatorname{Re}\left\langle\widehat{C}_{0} u_{1}, u_{1}\right\rangle+\operatorname{Re}\left\langle\tilde{A}_{\xi}\left(\sigma u^{(0)}\right), u_{1}\right\rangle \\
& \quad+\operatorname{Re}\left\langle\tilde{B}_{\xi}\left(\sigma u^{(0)}\right), u_{1}\right\rangle-\operatorname{Re}\left\{\left\langle Q_{0} \tilde{B}_{\xi}\left(\sigma u^{(0)}+u_{1}\right)\right\rangle\left\langle u^{(0)}, u_{1}\right\rangle\right\}=0 .
\end{aligned}
$$


We add $\left(\alpha_{0} / \gamma^{2}\right) \times(4.17)$ to $(4.19)$, to obtain

$$
\begin{aligned}
& \frac{1}{2} \frac{d}{d t}\left(\frac{\alpha_{0}}{\gamma^{2}}|\sigma|^{2}+E_{0}\left[u_{1}\right]\right)+\tilde{D}_{\xi}\left[w_{1}\right]+\operatorname{Re}\left\langle\widehat{C}_{0} u_{1}, u_{1}\right\rangle+\operatorname{Re}\left\langle\tilde{A}_{\xi}\left(\sigma u^{(0)}\right), u_{1}\right\rangle \\
& \quad+\operatorname{Re}\left\langle\tilde{B}_{\xi}\left(\sigma u_{1}^{(0)}, u_{1}\right)\right\rangle-\operatorname{Re}\left\{\left\langle Q_{0} \tilde{B}_{\xi}\left(\sigma u^{(0)}+u_{1}\right)\right\rangle\left\langle u^{(0)}, u_{1}\right\rangle\right\}=0,
\end{aligned}
$$

where $u_{1}^{(0)}={ }^{\mathrm{T}}\left(0, w^{(0)}\right)$. Here we used the equation

$$
-\operatorname{Re}\left\langle u_{1}, \tilde{B}_{\xi}\left(\sigma u_{0}^{(0)}\right)\right\rangle+\operatorname{Re}\left\langle\tilde{B}_{\xi}\left(\sigma u^{(0)}\right), u_{1}\right\rangle=\operatorname{Re}\left\langle\tilde{B}_{\xi}\left(\sigma u_{1}^{(0)}\right), u_{1}\right\rangle .
$$

By the Poincaré inequality we have

$$
\begin{aligned}
\left|\operatorname{Re}\left\langle\tilde{A}_{\xi}\left(\sigma u^{(0)}\right), u_{1}\right\rangle\right| & \leq C\left(\frac{v}{\gamma^{2}}|\xi|^{2}|\sigma|\left|w_{1}^{3}\right|_{2}+\frac{\tilde{v}}{\gamma^{2}}|\xi||\sigma|\left|\nabla^{\prime} \cdot w_{1}^{\prime}+i \xi w_{1}^{3}\right|_{2}\right) \\
& \leq \frac{1}{8} \tilde{D}_{\xi}\left[w_{1}\right]+C \frac{v+\tilde{v}}{\gamma^{4}}|\xi|^{2}|\sigma|^{2}, \\
\left|\operatorname{Re}\left\langle\tilde{B}_{\xi}\left(\sigma u_{1}^{(0)}\right), u_{1}\right\rangle\right| & \leq C|\xi||\sigma|\left(\frac{1}{\gamma^{2}}\left|\phi_{1}\right|_{2}+\frac{1}{\gamma^{2}}\left|w_{1}^{3}\right|_{2}\right) \\
& \leq C\left(\frac{1}{\gamma^{2}}+\frac{1}{v \gamma^{4}}\right)|\xi|^{2}|\sigma|^{2}+\frac{1}{\gamma^{2}}\left|\phi_{1}\right|_{2}^{2}+\frac{1}{8} \tilde{D}_{\xi}\left[w_{1}\right] .
\end{aligned}
$$

Since $\left\langle\phi_{1}\right\rangle=0$, it holds that

$$
\left|\left\langle u^{(0)}, u_{1}\right\rangle\right| \leq C \frac{1}{\gamma^{2}}\left|w_{1}^{3}\right|_{2} .
$$

Applying Lemma 4.6 and the Poincaré and Hölder inequalities, we thus have the following estimates:

$$
\begin{aligned}
\left|\operatorname{Re}\left\{\left\langle Q_{0} \tilde{B}_{\xi}\left(\sigma u^{(0)}+u_{1}\right)\right\rangle\left\langle u^{(0)}, u_{1}\right\rangle\right\}\right| \leq & C|\xi|\left(|\sigma|+\left|\phi_{1}\right|_{2}+\gamma^{2}\left|w_{1}^{3}\right|_{2}\right) \frac{1}{\gamma^{2}}\left|w_{1}^{3}\right|_{2} \\
\leq & \frac{1}{8} \tilde{D}_{\xi}\left[w_{1}\right] \\
& +C\left(\frac{1}{v \gamma^{4}}|\xi|^{2}|\sigma|^{2}+\frac{1}{v \gamma^{4}}\left|\phi_{1}\right|_{2}^{2}+\frac{1}{v} \tilde{D}_{\xi}\left[w_{1}\right]\right), \\
\left|\operatorname{Re}\left\{\left\langle\widehat{C}_{0} u_{1}, u_{1}\right\rangle\right\}\right| \leq & C\left(\frac{v}{\gamma^{4}}\left|\phi_{1}\right|_{2}^{2}+\frac{1}{v} \tilde{D}_{\xi}\left[w_{1}\right]\right) .
\end{aligned}
$$

Therefore, we find that there exists a constant $v_{0}>0$ such that if $v \geq v_{0}$, then

$$
\frac{1}{2} \frac{d}{d t}\left(\frac{\alpha_{0}}{\gamma^{2}}|\sigma|^{2}+E_{0}\left[u_{1}\right]\right)+\frac{1}{2} \tilde{D}_{\xi}\left[w_{1}\right] \leq C\left\{\left(\frac{1}{\gamma^{2}}+\frac{v+\tilde{v}}{\gamma^{4}}\right)|\xi|^{2}|\sigma|^{2}+\left(\frac{1}{\gamma^{2}}+\frac{v}{\gamma^{4}}\right)\left|\phi_{1}\right|_{2}^{2}\right\} .
$$

We next estimate $\dot{\phi}_{1}$. By the first equation of (4.11) it holds that

$$
\begin{aligned}
\frac{1}{\gamma^{2}} \dot{\phi}_{1}= & -\left(\nabla^{\prime} \cdot\left(\rho_{s} w_{1}^{\prime}\right)+i \xi \rho_{s} w_{1}^{3}\right) \\
& -\frac{1}{\gamma^{2}}\left\{i \xi v_{s}^{3} \sigma \phi^{(0)}+\gamma^{2} i \xi \rho_{s} \sigma w^{(0), 3}-\left\langle Q_{0} \tilde{B}_{\xi}\left(\sigma u^{(0)}+u_{1}\right)\right\rangle\right\} .
\end{aligned}
$$


We thus obtain

$$
\frac{1}{\gamma^{4}}\left|\dot{\phi}_{1}\right|_{2}^{2} \leq C\left\{\frac{1}{\gamma^{4}}|\xi|^{2}|\sigma|^{2}+\frac{1}{\gamma^{4}}|\xi|^{2}\left|\phi_{1}\right|_{2}^{2}+\left(\frac{1}{v+\tilde{v}}+\frac{\omega^{2}}{v}\right) \tilde{D}_{\xi}\left[w_{1}\right]\right\} .
$$

Multiplying by $v+\tilde{v}$ to both sides, we have the desired estimate. This completes the proof.

Let us estimate $\left|\phi_{1}\right|_{2}$. We first introduce the Bogovskii lemma.

PROPOSITION 4.8. Let $\dot{L^{2}}(D)$ be defined by

$$
\dot{L}^{2}(D)=\left\{f \in L^{2}(D):\langle f\rangle=0\right\} .
$$

There exists a bounded operator $\mathcal{B}: \dot{L}^{2}(D) \rightarrow H_{0}^{1}(D)$ such that

$$
\begin{gathered}
-\nabla^{\prime} \cdot \mathcal{B} f=f, \\
\left|\nabla^{\prime} \mathcal{B} f\right|_{2} \leq C|f|_{2}
\end{gathered}
$$

for any $f \in \dot{L^{2}}(D)$.

Proof. See, for example, [2, III.3, Theorem 3.2].

The proof of the following proposition is based on the argument in [4].

Proposition 4.9. There exist constants $v_{0}>0$ and $\omega_{0}>0$ such that if $v \geq v_{0}, \gamma \geq 1$ and $(v+\tilde{v}) \omega / v \leq \omega_{0}$, then the following estimates hold:

$$
\begin{aligned}
\frac{d}{d t} J_{0}\left[u_{1}\right]+\frac{1}{2} \frac{1}{v+\tilde{v}}\left|\phi_{1}\right|_{2}^{2} \leq & C\left\{\left(\frac{\gamma^{2}}{v(v+\tilde{v})}+1\right) \tilde{D}_{\xi}\left[w_{1}\right]+\frac{v}{v+\tilde{v}}|\xi|^{2} \tilde{D}_{\xi}\left[w_{1}\right]\right. \\
+ & \left.\left(\frac{1}{\gamma^{2}}+\frac{v+\tilde{v}}{\gamma^{4}}\right)|\xi|^{2}|\sigma|^{2}\right\} \\
\left|J_{0}\left[u_{1}\right]\right| \leq & C\left\{\frac{\gamma^{2}}{v(v+\tilde{v})}\left|w_{1}\right|_{2}^{2}+\frac{v}{\gamma^{2}(v+\tilde{v})}\left|\phi_{1}\right|_{2}^{2}\right\}
\end{aligned}
$$

where

$$
J_{0}\left[u_{1}\right]=\frac{1}{v+\tilde{v}} \operatorname{Re}\left(w_{1}^{\prime}, \rho_{s} \psi^{\prime}\right)
$$

with $\psi^{\prime}=\mathcal{B} \phi_{1}$.

Proof. Set $\psi^{\prime}=\mathcal{B} \phi_{1}$. Taking the inner product of $(4.11)_{2}$ with $\rho_{s} \psi^{\prime}$, we get

$$
\operatorname{Re}\left(\partial_{t} w_{1}^{\prime}, \rho_{s} \psi^{\prime}\right)+\operatorname{Re}\left(\nabla^{\prime}\left(\frac{P^{\prime}\left(\rho_{s}\right)}{\gamma^{2} \rho_{s}} \phi_{1}\right), \rho_{s} \psi^{\prime}\right)=\operatorname{Re} I,
$$

where

$$
\begin{aligned}
I= & -v\left(\nabla^{\prime} w_{1}^{\prime}, \nabla^{\prime} \psi^{\prime}\right)-v \xi^{2}\left(w_{1}^{\prime}, \psi^{\prime}\right)+\tilde{v}\left(\nabla^{\prime} \cdot w_{1}^{\prime}, \phi_{1}\right)+\tilde{v} i \xi\left(w_{1}^{3}, \phi_{1}\right) \\
& -i \xi\left(\rho_{s} v_{s}^{3} w_{1}^{\prime}, \psi^{\prime}\right)+\tilde{v} i \xi\left(\sigma w^{(0), 3}, \phi_{1}\right) .
\end{aligned}
$$

Let us estimate the first term of the left-hand side of (4.22). It holds that

$$
\operatorname{Re}\left(\partial_{t} w_{1}^{\prime}, \rho_{s} \psi^{\prime}\right)=\frac{d}{d t} \operatorname{Re}\left(w_{1}^{\prime}, \rho_{s} \psi^{\prime}\right)-\operatorname{Re}\left(w_{1}^{\prime}, \rho_{s} \partial_{t} \psi^{\prime}\right)
$$


Since

$$
-\nabla^{\prime} \cdot \partial_{t} \psi^{\prime}=\partial_{t} \phi_{1}
$$

and

$$
\begin{aligned}
\partial_{t} \phi_{1}=- & \left\{i \xi v_{s}^{3} \phi_{1}+\gamma^{2} \nabla^{\prime} \cdot\left(\rho_{s} w_{1}^{\prime}\right)+i \xi \gamma^{2} \rho_{s} w_{1}^{3}\right. \\
& \left.+i \xi v_{s}^{3} \sigma \phi^{(0)}+\gamma^{2} i \xi \rho_{s} \sigma w^{(0), 3}-\left\langle Q_{0} \tilde{B}_{\xi}\left(\sigma u^{(0)}+u_{1}\right)\right\rangle \phi^{(0)}\right\},
\end{aligned}
$$

we obtain

$$
\begin{aligned}
\left|\operatorname{Re}\left(w_{1}^{\prime}, \rho_{s} \partial_{t} \psi^{\prime}\right)\right| \leq & C\left|w_{1}^{\prime}\right|_{2}\left|\partial_{t} \psi^{\prime}\right|_{2} \\
\leq & C\left|w_{1}^{\prime}\right|_{2}\left\{|\xi|\left|\phi_{1}\right|_{2}+\gamma^{2}\left|\nabla^{\prime} \cdot w_{1}^{\prime}\right|_{2}+\gamma^{2}\left|w_{1}^{\prime}\right|_{2}+\gamma^{2}|\xi|^{2}\left|w_{1}^{3}\right|_{2}+|\xi||\sigma|\right\} \\
\leq & \frac{1}{8}\left|\phi_{1}\right|_{2}^{2}+C\left\{\left(\gamma^{2}+\frac{\gamma^{2}}{v+\tilde{v}}\right)\left|w_{1}\right|_{2}^{2}+\left(1+\gamma^{2}\right)|\xi|^{2}\left|w_{1}\right|_{2}^{2}+\gamma^{2}\left|\nabla^{\prime} \cdot w_{1}^{\prime}\right|_{2}^{2}\right. \\
& \left.+\frac{v+\tilde{v}}{\gamma^{2}}|\xi|^{2}|\sigma|^{2}\right\} \\
\leq & \frac{1}{8}\left|\phi_{1}\right|_{2}^{2}+C\left\{\left(\frac{1}{v}+\frac{\gamma^{2}}{v}+\frac{\gamma^{2}}{v(v+\tilde{v})}\right) \tilde{D}_{\xi}\left[w_{1}\right]+\frac{v+\tilde{v}}{\gamma^{2}}|\xi|^{2}|\sigma|^{2}\right\} .
\end{aligned}
$$

We next estimate the second term of the left-hand side of (4.22). There exists $\omega_{0}>0$ such that if $\omega \leq \omega_{0}$, then it holds that

$$
\begin{aligned}
\operatorname{Re}\left(\nabla^{\prime}\left(\frac{P^{\prime}\left(\rho_{s}\right)}{\gamma^{2} \rho_{s}} \phi_{1}\right), \rho_{s} \psi^{\prime}\right) & =\left|\sqrt{\frac{P^{\prime}\left(\rho_{s}\right)}{\gamma^{2}}} \phi_{1}\right|_{2}^{2}-\operatorname{Re}\left(\frac{P^{\prime}\left(\rho_{s}\right)}{\gamma^{2} \rho_{s}} \phi_{1},\left(\nabla^{\prime} \rho_{s}\right) \cdot \psi^{\prime}\right) \\
& \geq C(1-\omega)\left|\sqrt{\frac{P^{\prime}\left(\rho_{s}\right)}{\gamma^{2}}} \phi_{1}\right|_{2}^{2} \\
& \geq \frac{3}{4}\left|\phi_{1}\right|_{2}^{2} .
\end{aligned}
$$

As for $I$, we have

$$
|I| \leq \frac{1}{8}\left|\phi_{1}\right|_{2}^{2}+C\left\{\left(v+\frac{\tilde{v}^{2}}{v+\tilde{v}}+\frac{1}{v}\right) \tilde{D}_{\xi}\left[w_{1}\right]+v|\xi|^{2} \tilde{D}_{\xi}\left[w_{1}\right]+\frac{\tilde{v}^{2}}{\gamma^{4}}|\xi|^{2}|\sigma|^{2}\right\} .
$$

Therefore, it holds that

$$
\begin{aligned}
\frac{d}{d t} \operatorname{Re}\left(w_{1}^{\prime}, \rho_{s} \psi^{\prime}\right)+\frac{1}{2}\left|\phi_{1}\right|_{2}^{2} \leq & C\left\{\left(\frac{1}{v}+\frac{\gamma^{2}}{v}+\frac{\gamma^{2}}{v(v+\tilde{v})}+v+\frac{\tilde{v}^{2}}{v+\tilde{v}}\right) \tilde{D}_{\xi}\left[w_{1}\right]\right. \\
& \left.+v|\xi|^{2} \tilde{D}_{\xi}\left[w_{1}\right]+\left(\frac{v+\tilde{v}}{\gamma^{2}}+\frac{(v+\tilde{v})^{2}}{\gamma^{4}}\right)|\xi|^{2}|\sigma|^{2}\right\} .
\end{aligned}
$$

Multiplying both sides of this inequality by $1 /(v+\tilde{v})$, we have the desired estimate. This completes the proof.

We next derive the estimate for $\sigma$. We introduce a notation. Let us define $J_{1}[u]$ by

$$
J_{1}[u]=\operatorname{Re}\left\{i \xi \frac{1}{v+\tilde{v}}\left\langle\rho_{s}\left(A+|\xi|^{2}\right)^{-1}\left[\rho_{s} w_{1}^{3}\right]\right\rangle \bar{\sigma}\right\}
$$


for $u=\sigma u^{(0)}+u_{1}$ with $u_{1}={ }^{\mathrm{T}}\left(\phi_{1}, w_{1}^{1}, w_{1}^{2}, w_{1}^{3}\right)$. Here $A$ is an operator on $L^{2}(D)$ defined by

$$
A \varphi=-\Delta^{\prime} \varphi \text { for } \varphi \in D(A)=H^{2}(D) \cap H_{0}^{1}(D) .
$$

PROPOSITION 4.10. There exist constants $\nu_{0}>0, \gamma_{0}>0, \omega_{0}>0$ and $\tilde{\alpha}_{0}>0$ such that if $v \geq v_{0}, \gamma^{2} /(v+\tilde{v}) \geq \gamma_{0}^{2}$ and $(v+\tilde{v}) \omega / v \leq \omega_{0}$, then the following estimates hold:

$$
\begin{gathered}
\frac{1}{2} \frac{d}{d t}\left(\frac{v}{(v+\tilde{v}) \gamma^{2}}|\sigma|^{2}+J_{1}[u]\right)+\frac{1}{2} \frac{\tilde{\alpha}_{0}}{v+\tilde{v}}|\xi|^{2}|\sigma|^{2} \\
\leq C\left\{\frac{v}{(v+\tilde{v}) \gamma^{2}}\left|\phi_{1}\right|_{2}^{2}+\frac{1}{v+\tilde{v}}|\xi|^{2}\left|\phi_{1}\right|_{2}^{2}+\frac{v^{2}}{(v+\tilde{v}) \gamma^{4}} \max \left\{1,|\xi|^{2}\right\}|\xi|^{2}\left|\phi_{1}\right|_{2}^{2}\right. \\
\left.\quad+\frac{\gamma^{2}}{(v+\tilde{v}) v} \tilde{D}_{\xi}\left[w_{1}\right]+\left(\frac{\tilde{v}^{2}}{(v+\tilde{v})^{2}}+\frac{1}{v}\right)|\xi|^{2} \tilde{D}_{\xi}\left[w_{1}\right]\right\}, \\
\left|J_{1}[u]\right| \leq \frac{1}{\gamma^{2}}|\sigma|^{2}+C \frac{\gamma^{2}}{(v+\tilde{v})^{2}}\left|w_{1}\right|_{2}^{2},
\end{gathered}
$$

where $\tilde{\alpha}_{0}$ is a positive constant.

Proof. Since

$$
\left\langle Q_{0} \tilde{B}_{\xi}\left(\sigma u^{(0)}+u_{1}\right)\right\rangle=\left\langle Q_{0} \tilde{B}_{\xi} u^{(0)}\right\rangle \sigma+\gamma^{2} i \xi\left\langle\rho_{s} w_{1}^{3}\right\rangle+i \xi\left\langle v_{s}^{3} \phi_{1}\right\rangle,
$$

equation (4.8) is written as

$$
\partial_{t} \sigma+\left\langle Q_{0} \tilde{B}_{\xi} u^{(0)}\right\rangle \sigma+\gamma^{2} i \xi\left\langle\rho_{s} w_{1}^{3}\right\rangle=-i \xi\left\langle v_{s}^{3} \phi_{1}\right\rangle .
$$

Set

$$
\tilde{B}_{\xi}^{3}=\left(i \xi \frac{P^{\prime}\left(\rho_{s}\right)}{\gamma^{2} \rho_{s}} \quad 0 \quad i \xi v_{s}^{3}\right) .
$$

Since $\left(P^{\prime}\left(\rho_{s}\right) / \gamma^{2} \rho_{S}\right) \phi^{(0)}=\alpha_{0}$, we have

$$
\tilde{B}_{\xi}^{3} u_{0}^{(0)}=i \xi \frac{P^{\prime}\left(\rho_{s}\right)}{\gamma^{2} \rho_{s}} \sigma \phi^{(0)}=i \xi \alpha_{0} .
$$

We thus obtain

$$
-\left(\Delta^{\prime}-\xi^{2}\right) w_{1}^{3}=-\frac{\alpha_{0}}{v} i \xi \sigma \rho_{s}-\frac{\rho_{s}}{v} \partial_{t} w_{1}^{3}+I_{1}^{3} .
$$

Here

$$
\begin{aligned}
I_{1}^{3}=-\frac{\rho_{s}}{v}\left\{\widehat{C}_{0}^{3} u_{1}-\frac{\tilde{v}}{\rho_{s}} i \xi\left(\nabla^{\prime} \cdot w_{1}^{\prime}+i \xi w_{1}^{3}\right)+\tilde{B}_{\xi}^{3} u_{1}+\sigma \tilde{M}_{\xi}^{3} u_{1}^{(0)}\right. \\
\left.-\left\langle Q_{0} \tilde{B}_{\xi}\left(\sigma u^{(0)}+u_{1}\right)\right\rangle w^{(0), 3}\right\}
\end{aligned}
$$

where $\widehat{C}_{0}^{3}$ and $\tilde{M}_{\xi}^{3}$ are $1 \times 4$ matrix operators defined by

$$
\tilde{M}_{\xi}^{3}=\left(\begin{array}{lll}
0 & 0 & \frac{v+\tilde{v}}{\rho_{s}} \xi^{2}
\end{array}\right)+\tilde{B}_{\xi}^{3}, \quad \widehat{C}_{0}^{3}=\left(\begin{array}{ccc}
\frac{v}{\gamma^{2} \rho_{s}^{2}} \Delta^{\prime} v_{s}^{3} & \mathrm{~T}\left(\nabla^{\prime} v_{s}^{3}\right) & 0
\end{array}\right) .
$$

It then follows from (4.25) that

$$
w_{1}^{3}=-\frac{\alpha_{0}}{v} i \xi \sigma\left(A+|\xi|^{2}\right)^{-1} \rho_{s}-\left(A+|\xi|^{2}\right)^{-1}\left[\frac{\rho_{s}}{v} \partial_{t} w_{1}^{3}\right]+\left(A+|\xi|^{2}\right)^{-1} I_{1}^{3}
$$


Substituting this into (4.24) we obtain

$$
\partial_{t} \sigma+\left\langle Q_{0} \tilde{B}_{\xi} u^{(0)}\right\rangle \sigma+\frac{\alpha_{0} \gamma^{2}}{v}\left\langle\rho_{s}\left(A+|\xi|^{2}\right)^{-1} \rho_{s}\right\rangle|\xi|^{2} \sigma=I_{1}^{0}-I_{2}^{0},
$$

where

$$
\begin{aligned}
& I_{1}^{0}=-\gamma^{2} i \xi\left\langle\rho_{s}\left(A+|\xi|^{2}\right)^{-1} I_{1}^{3}\right\rangle-i \xi\left\langle v_{s}^{3} \phi_{1}\right\rangle, \\
& I_{2}^{0}=\gamma^{2} i \xi\left\langle\rho_{s}\left(A+|\xi|^{2}\right)^{-1}\left[\frac{\rho_{s}}{v} \partial_{t} w_{1}^{3}\right]\right\rangle .
\end{aligned}
$$

Let us calculate (4.26) $\times \bar{\sigma}$ and take its real part. Since $\operatorname{Re}\left\{\left\langle Q_{0} \tilde{B}_{\xi} u^{(0)}\right\rangle\right\}=0$, we have

$$
\frac{1}{2} \frac{d}{d t}|\sigma|^{2}+\frac{\alpha_{0} \gamma^{2}}{v}\left\langle\rho_{s}\left(A+|\xi|^{2}\right)^{-1} \rho_{s}\right\rangle|\xi|^{2}|\sigma|^{2}=\operatorname{Re}\left(I_{1}^{0} \bar{\sigma}\right)+\operatorname{Re}\left(I_{2}^{0} \bar{\sigma}\right) .
$$

Since $\left\langle\rho_{s}\left(A+|\xi|^{2}\right)^{-1} \rho_{S}\right\rangle=\left|\left(A+|\xi|^{2}\right)^{-1 / 2} \rho_{S}\right|_{2}^{2}$ is continuous in $\xi$ and is positive for all $\xi \in \mathbb{R}$, we see that there exists a positive constant $\tilde{\alpha}_{0}=\mathcal{O}\left(|\xi|^{-2}\right)$ as $|\xi| \rightarrow \infty$ such that

$$
\frac{\alpha_{0} \gamma^{2}}{v}\left\langle\rho_{s}\left(A+|\xi|^{2}\right)^{-1} \rho_{s}\right\rangle \geq \frac{\tilde{\alpha}_{0} \gamma^{2}}{v}
$$

for all $\xi \in \mathbb{R}$ with $|\xi| \leq R$. We thus obtain

$$
\frac{1}{2} \frac{d}{d t}|\sigma|^{2}+\frac{\tilde{\alpha}_{0} \gamma^{2}}{v}|\xi|^{2}|\sigma|^{2} \leq \operatorname{Re}\left(I_{1}^{0} \bar{\sigma}\right)+\operatorname{Re}\left(I_{2}^{0} \bar{\sigma}\right) .
$$

As for the right-hand side of (4.27), we see from

$$
\left|\left(A+|\xi|^{2}\right)^{-1} p\right|_{2} \leq \frac{C}{|\xi|^{2}+1}|p|_{2}
$$

that

$$
\begin{aligned}
\left|\operatorname{Re}\left(I_{1}^{0} \bar{\sigma}\right)\right| \leq & \frac{\tilde{\alpha}_{0} \gamma^{2}}{v}\left(\frac{1}{10}+C \frac{1}{\gamma^{2}}\right) \min \left\{1,|\xi|^{2}\right\}|\sigma|^{2} \\
& +C\left\{\left|\phi_{1}\right|_{2}^{2}+\left(\frac{\gamma^{2}}{v}+\frac{1}{v \gamma^{2}}\right)|\xi|^{2}\left|\phi_{1}\right|_{2}^{2}+\frac{v}{\gamma^{2}} \max \left\{1,|\xi|^{2}\right\}|\xi|^{2}\left|\phi_{1}\right|_{2}^{2}\right. \\
& \left.+\frac{\gamma^{2}}{v^{2}} \tilde{D}_{\xi}\left[w_{1}\right]+\left(\frac{(v+\tilde{v}) \gamma^{2}}{v^{2}}+\frac{\gamma^{2} \tilde{v}^{2}}{(v+\tilde{v}) v}\right)|\xi|^{2} \tilde{D}_{\xi}\left[w_{1}\right]\right\} .
\end{aligned}
$$

We next derive the estimate for $I_{2}^{0} \bar{\sigma}$. It holds that

$$
\begin{aligned}
\operatorname{Re}\left(I_{2}^{0} \bar{\sigma}\right)= & \operatorname{Re}\left\{\gamma^{2} i \xi\left\langle\rho_{s}\left(A+|\xi|^{2}\right)^{-1}\left(\frac{\rho_{s}}{v} \partial_{t} w_{1}^{3}\right)\right) \bar{\sigma}\right\} \\
= & \frac{d}{d t} \operatorname{Re}\left\{i \xi \frac{\gamma^{2}}{v}\left\langle\rho_{s}\left(A+|\xi|^{2}\right)^{-1}\left(\rho_{s} w_{1}^{3}\right)\right\rangle \bar{\sigma}\right\} \\
& -\operatorname{Re}\left\{i \xi \frac{\gamma^{2}}{v}\left\langle\rho_{s}\left(A+|\xi|^{2}\right)^{-1}\left(\rho_{s} w_{1}^{3}\right)\right\rangle \partial_{t} \bar{\sigma}\right\} \\
= & \frac{d}{d t}\left(\frac{\gamma^{2}(v+\tilde{v})}{v} J_{1}[u]\right)-\operatorname{Re}\left\{i \xi \frac{\gamma^{2}}{v}\left\langle\rho_{s}\left(A+|\xi|^{2}\right)^{-1}\left(\rho_{s} w_{1}^{3}\right)\right\rangle \partial_{t} \bar{\sigma}\right\}
\end{aligned}
$$


Let us estimate the second term of the right-hand side of this equation. We see from (4.8) that

$$
\begin{aligned}
& \left|\operatorname{Re}\left\{i \xi \frac{\gamma^{2}}{v}\left\langle\rho_{s}\left(A+|\xi|^{2}\right)^{-1}\left[\rho_{s} w_{1}^{3}\right]\right\rangle \partial_{t} \bar{\sigma}\right\}\right| \\
& \quad=\left|\operatorname{Re}\left\{i \xi \frac{\gamma^{2}}{v}\left\langle\rho_{s}\left(A+|\xi|^{2}\right)^{-1}\left(\rho_{s} w_{1}^{3}\right)\right\rangle\left\{-\left\langle Q_{0} \tilde{B}_{\xi} u^{(0)}\right\rangle-\gamma^{2} i \xi\left\langle\rho_{s} w_{1}^{3}\right\rangle-i \xi\left\langle v_{s}^{3} \phi_{1}\right\rangle\right\}\right\}\right| \\
& \quad \leq C \frac{\gamma^{2}}{v} \frac{|\xi|}{1+|\xi|^{2}}\left|w_{1}\right|_{2}\left\{|\xi||\sigma|+\gamma^{2}|\xi|\left|w_{1}\right|_{2}+|\xi||\phi|_{2}\right\} \\
& \quad \leq \frac{1}{10} \frac{\tilde{\alpha}_{0} \gamma^{2}}{v} \min \left\{1,|\xi|^{2}\right\}|\sigma|^{2}+C\left\{\frac{1}{v}|\xi|^{2}\left|\phi_{1}\right|_{2}^{2}+\frac{\gamma^{4}}{v^{2}} \tilde{D}_{\xi}\left[w_{1}\right]\right\} .
\end{aligned}
$$

If $1 / \nu, 1 / \gamma^{2}$ and $(v+\tilde{v}) / \gamma^{2}$ are sufficiently small, it then follows from (4.27)-(4.30) that

$$
\begin{aligned}
\frac{1}{2} \frac{d}{d t}\left(|\sigma|^{2}+\frac{\gamma^{2}(v+\tilde{v})}{v} J_{1}[u]\right)+\frac{1}{2} \frac{\tilde{\alpha}_{0} \gamma^{2}}{v}|\xi|^{2}|\sigma|^{2} \\
\leq C\left\{\left|\phi_{1}\right|_{2}^{2}+\frac{\gamma^{2}}{v}|\xi|^{2}\left|\phi_{1}\right|_{2}^{2}+\frac{v}{\gamma^{2}} \max \left\{1,|\xi|^{2}\right\}|\xi|^{2}\left|\phi_{1}\right|_{2}^{2}\right. \\
\left.\quad+\frac{\gamma^{4}}{v^{2}} \tilde{D}_{\xi}\left[w_{1}\right]+\left(\frac{\gamma^{2}(v+\tilde{v})}{v^{2}}+\frac{\gamma^{2} \tilde{v}^{2}}{v(v+\tilde{v})}\right)|\xi|^{2} \tilde{D}_{\xi}\left[w_{1}\right]\right\} .
\end{aligned}
$$

Furthermore, we have the estimate

$$
\begin{aligned}
\left|J_{1}[u]\right| & =\left|\operatorname{Re}\left(i \xi \frac{1}{v+\tilde{v}}\left\langle\rho_{s}\left(A+|\xi|^{2}\right)^{-1}\left[\rho_{s} w_{1}^{3}\right]\right\rangle \bar{\sigma}\right)\right| \\
& \leq \frac{1}{\gamma^{2}}|\sigma|^{2}+C \frac{\gamma^{2}}{(v+\tilde{v})^{2}}\left|w_{1}\right|_{2}^{2} .
\end{aligned}
$$

Multiplying both sides of (4.31) by $v /\left(\gamma^{2}(v+\tilde{v})\right)$, we obtain the desired estimates. This completes the proof.

From Propositions 4.7, 4.9 and 4.10, we get the estimate of $|\sigma|,\left|\phi_{1}\right|_{2}$ and $\left|w_{1}\right|_{2}$.

Proposition 4.11. Let $R>0$. There exist positive constants $v_{0}, \gamma_{0}$, $\omega_{0}$ independent of $R$ and an energy functional $E_{1}[u]$ such that if $v \geq v_{0} R^{2}, \gamma^{2} /(v+\tilde{v}) \geq \gamma_{0}^{2} R^{2},(v+\tilde{v}) \omega / v \leq \omega_{0}$ and $|\xi| \leq R$, then the following estimates hold:

$$
\begin{gathered}
\frac{d}{d t} E_{1}[u]+\frac{1}{v+\tilde{v}}\left(|\xi|^{2}|\sigma|^{2}+\left|\phi_{1}\right|_{2}^{2}\right)+\tilde{D}_{\xi}\left[w_{1}\right] \leq 0, \\
\frac{1}{2}\left(\frac{1}{\gamma^{2}}|\sigma|^{2}+E_{0}\left[u_{1}\right]\right) \leq C E_{1}[u] \leq \frac{3}{2}\left(\frac{1}{\gamma^{2}}|\sigma|^{2}+E_{0}\left[u_{1}\right]\right),
\end{gathered}
$$

where $C$ is a positive constant independent of $u$.

Proof. For a given $R>0$ we assume that $|\xi| \leq R$. Let $b_{1}>1$ and $b_{2}>1$ be constants. Define $E_{1}[u]$ by

$$
E_{1}[u]=b_{1}\left(1+\frac{\gamma^{2}}{v(v+\tilde{v})}\right)\left(\frac{\alpha_{0}}{\gamma^{2}}|\sigma|^{2}+E_{0}\left[u_{1}\right]\right)+b_{2} J_{0}\left[u_{1}\right]+\frac{v}{\gamma^{2}(v+\tilde{v})}|\sigma|^{2}+J_{1}[u] .
$$


Since we have

$$
\begin{gathered}
\frac{1}{2}\left(\left|\phi_{1}\right|_{2}^{2}+\left|w_{1}\right|_{2}^{2}\right) \leq C_{0} E_{0}\left[u_{1}\right] \leq \frac{3}{2}\left(\left|\phi_{1}\right|_{2}^{2}+\left|w_{1}\right|_{2}^{2}\right), \\
\left|J_{0}\left[u_{1}\right]\right| \leq C_{1}\left\{\frac{v}{\gamma^{2}(v+\tilde{v})}\left|\phi_{1}\right|_{2}^{2}+\frac{\gamma^{2}}{v(v+\tilde{v})}\left|w_{1}\right|_{2}^{2}\right\} \\
\left|J_{1}[u]\right| \leq \frac{1}{\gamma^{2}}|\sigma|^{2}+C_{2} \frac{\gamma^{2}}{(v+\tilde{v})^{2}}\left|w_{1}\right|_{2}^{2},
\end{gathered}
$$

if $1 /(v+\tilde{v})<1,(v+\tilde{v}) / \gamma^{2}<1$ and $b_{1}>8 \max \left\{C_{0} C_{1} b_{2}, C_{0} C_{2}, \alpha_{0}^{-1}\right\}$, then there exists a constant $C>0$ such that

$$
\frac{1}{2}\left(\frac{1}{\gamma^{2}}|\sigma|^{2}+E_{0}\left[u_{1}\right]\right) \leq C E_{1}[u] \leq \frac{3}{2}\left(\frac{1}{\gamma^{2}}|\sigma|^{2}+E_{0}\left[u_{1}\right]\right) .
$$

Let us compute $b_{1} \times\left(1+\gamma^{2} / v(v+\tilde{v})\right) \times(4.12)+b_{2} \times(4.21)+(4.23)$ then

$$
\begin{aligned}
& \frac{1}{2} \frac{d}{d t} E_{1}[u]+\frac{b_{1}}{2}\left(1+\frac{\gamma^{2}}{v(v+\tilde{v})}\right) \tilde{D}_{\xi}\left[w_{1}\right]+\frac{b_{2}}{2} \frac{1}{v+\tilde{v}}\left|\phi_{1}\right|_{2}^{2}+\frac{\tilde{\alpha}_{0}}{2} \frac{1}{v+\tilde{v}}|\xi|^{2}|\sigma|^{2} \\
& \leq C_{3}\left\{b_{1}\left(1+\frac{\gamma^{2}}{v(v+\tilde{v})}\right)\left(\frac{1}{\gamma^{2}}+\frac{v+\tilde{v}}{\gamma^{4}}\right)|\xi|^{2}|\sigma|^{2}\right. \\
&+b_{1}\left(1+\frac{\gamma^{2}}{v(v+\tilde{v})}\right)\left(\frac{1}{\gamma^{2}}+\frac{v}{\gamma^{4}}\right)\left|\phi_{1}\right|_{2}^{2}+b_{2}\left(\frac{1}{\gamma^{2}}+\frac{v+\tilde{v}}{\gamma^{4}}\right)|\xi|^{2}|\sigma|^{2} \\
&+b_{2}\left(1+\frac{\gamma^{2}}{v(v+\tilde{v})}\right) \tilde{D}_{\xi}\left[w_{1}\right]+b_{2} \frac{v}{v+\tilde{v}}|\xi|^{2} \tilde{D}_{\xi}\left[w_{1}\right] \\
&+\frac{v}{(v+\tilde{v}) \gamma^{2}}\left|\phi_{1}\right|_{2}^{2}+\frac{1}{v+\tilde{v}}|\xi|^{2}\left|\phi_{1}\right|_{2}^{2}+\frac{v^{2}}{(v+\tilde{v}) \gamma^{4}} \max \left\{1,|\xi|^{2}\right\}|\xi|^{2}\left|\phi_{1}\right|_{2}^{2} \\
&\left.+\frac{\gamma^{2}}{v(v+\tilde{v})} \tilde{D}_{\xi}\left[w_{1}\right]+\left(\frac{\tilde{v}^{2}}{(v+\tilde{v})^{2}}+\frac{1}{v}\right)|\xi|^{2} \tilde{D}_{\xi}\left[w_{1}\right]\right\} .
\end{aligned}
$$

Fix $b_{1}>1$ and $b_{2}>1$ so large that $b_{2} \geq 16 C_{3} R^{2}$ and $b_{1} \geq 16 \max \left\{C_{0} C_{1} b_{2}, C_{0} C_{2}, \alpha_{0}^{-1}\right.$, $\left.C_{3} b_{2}, C_{3} R^{2}\right\}$. We assume that $v \geq v_{0}$ and $\gamma \geq \gamma_{0}$ are so large that

$$
v \geq 16 C_{3} b_{1} \max \left\{\tilde{\alpha}_{0}^{-1}, b_{2}^{-1}, 1\right\}
$$

and

$$
\gamma^{2}>16 C_{3}\left(1+\tilde{\alpha}^{-1}+\tilde{\alpha}^{-1 / 2}\right)(v+\tilde{v}) \max \left\{b_{1}, b_{2}, b_{2}^{-1}\left(1+R^{2}\right)\right\} .
$$

It then follows that there exists a constant $C>0$ such that

$$
\frac{d}{d t} E_{1}[u]+C\left\{\frac{1}{v+\tilde{v}}|\xi|^{2}|\sigma|^{2}+\frac{1}{v+\tilde{v}}\left|\phi_{1}\right|_{2}^{2}+\tilde{D}_{\xi}\left[w_{1}\right]\right\} \leq 0 .
$$

We thus obtain the desired estimates. This completes the proof.

We are now in a position to prove the estimate of the $L^{2}$ norm of $U_{1}(t) u_{0}$. Before proceeding further we introduce some notation. For $R>0$ we define $\chi_{(R)}$ by $\chi_{(R)}(\xi)=1$ for $|\xi| \leq R$ and $\chi_{(R)}(\xi)=0$ for $|\xi|>R$. 
PROPOSITION 4.12. Let $R>0$. There exist positive constants $\nu_{0}, \gamma_{0}$ and $\omega_{0}$ such that if $v \geq v_{0} R^{2}, \gamma^{2} /(v+\tilde{v}) \geq \gamma_{0}^{2} R^{2}$, and $(v+\tilde{v}) \omega / v \leq \omega_{0}$, then for any $l=0,1, \ldots$, there exists a constant $C=C(l)>0$ such that the estimate

$$
\left\|\partial_{x_{3}}^{l} \mathcal{F}^{-1}\left[\chi_{(R)} e^{-t \widehat{L}_{\xi}} \widehat{u}_{0}\right]\right\|_{L^{2}} \leq C(1+t)^{-1 / 4-l / 2}\left\|u_{0}\right\|_{L^{1}\left(\mathbb{R}: L^{2}(D)\right)}
$$

holds for $t \geq 0$.

Proof. For a given $R>0$, we assume that $|\xi| \leq R$. Since

$$
|\xi|^{2}|\sigma|^{2}+\left|\phi_{1}\right|_{2}^{2}+\tilde{D}_{\xi}\left[w_{1}\right] \geq \tilde{d}_{0}|\xi|^{2}\left(|\sigma|^{2}+\left|\phi_{1}\right|_{2}^{2}+\left|w_{1}\right|_{2}^{2}\right)
$$

for some constant $\tilde{d}_{0}=\tilde{d}_{0}(R)>0$, we see from (4.33) that there exists a constant $d_{0}>0$ such that

$$
\frac{d}{d t} E_{1}[u](t)+d_{0}|\xi|^{2}|u|_{2}^{2} \leq 0 .
$$

This implies that

$$
\left|e^{-t \widehat{L}_{\xi}} \widehat{u}_{0}(\xi)\right|_{L^{2}} \leq C e^{-d_{0}|\xi|^{2} t}\left|\widehat{u}_{0}(\xi)\right|_{L^{2}}
$$

We thus obtain the desired estimate. This completes the proof.

We next estimate derivatives of $u$. We introduce some notation. We define $J_{2}^{(0)}[u]$ by

$$
J_{2}^{(0)}[u]=-2 \operatorname{Re}\left\langle\sigma u^{(0)}+u_{1}, \widehat{B} \xi \tilde{Q} u_{1}\right\rangle \quad \text { for } u=\sigma u^{(0)}+u_{1} .
$$

In addition, we set

$$
\begin{aligned}
& E_{2}^{(0)}[u]=\left(1+\frac{b_{3} \gamma^{2}}{v}\right)\left(\frac{\alpha_{0}}{\gamma^{2}}|\sigma|^{2}+E_{0}\left[u_{1}\right]\right)+\tilde{D}_{\xi}\left[w_{1}\right], \\
& \tilde{E}_{2}^{(0)}[u]=E_{2}^{(0)}[u]+J_{2}^{(0)}[u],
\end{aligned}
$$

where $b_{3}$ is a positive constant to be determined later. We note that there exists a constant $b_{3}^{*}>0$ such that if $b_{3} \geq b_{3}^{*}$ and $\gamma^{2} \geq 1$ then

$$
\frac{1}{2} E_{2}^{(0)}[u] \leq \tilde{E}_{2}^{(0)}[u] \leq \frac{3}{2} E_{2}^{(0)}[u] .
$$

Taking $b_{3}$ suitably large, we have the following estimate for $\tilde{E}_{2}^{(0)}[u]$.

PROPOSITION 4.13. There exist constants $b_{3} \geq b_{3}^{*}, v_{0}>0$ and $\omega_{0}>0$ such that if $v \geq v_{0}$, $\gamma^{2} \geq 1$ and $\omega \leq \omega_{0}$, then the following estimate holds:

$$
\begin{aligned}
\frac{1}{2} \frac{d}{d t} \tilde{E}_{2}^{(0)}[u]+\frac{1}{4} b_{3} \frac{\gamma^{2}}{v} \tilde{D}_{\xi}\left[w_{1}\right]+\frac{1}{2}\left|\sqrt{\rho_{s}} \partial_{t} w_{1}\right|_{2}^{2} \\
\leq C\left\{\left(\frac{1}{v}+\frac{v+\tilde{v}}{v \gamma^{2}}+\frac{\tilde{v}^{2}}{\gamma^{4}}\right)|\xi|^{2}|\sigma|^{2}+\frac{(v+\tilde{v})^{2}}{\gamma^{4}}|\xi|^{4}|\sigma|^{2}\right. \\
\left.+\left(\frac{1}{v}+\frac{1}{\gamma^{2}}+\frac{v^{2}}{\gamma^{4}}\right)\left|\phi_{1}\right|_{2}^{2}+\frac{1}{\gamma^{2}}|\xi|^{2}\left|\phi_{1}\right|_{2}^{2}\right\} .
\end{aligned}
$$


Proof. Since $u$ is a solution of

$$
\partial_{t} u+\widehat{L}_{\xi} u=0
$$

it holds that

$$
\left\langle\partial_{t} u, \partial_{t} \tilde{Q} u_{1}\right\rangle+\left\langle\widehat{L}_{\xi} u, \partial_{t} \tilde{Q} u_{1}\right\rangle=0 .
$$

We first consider the first term on the left-hand side of (4.39). Since

$$
\begin{gathered}
\partial_{t} \sigma=-\left\langle Q_{0} \tilde{B}_{\xi}\left(\sigma u^{(0)}+u_{1}\right)\right\rangle, \\
\left\langle u^{(0)}, \partial_{t} \tilde{Q} u_{1}\right\rangle=\left\langle u_{1}^{(0)}, \partial_{t} \tilde{Q} u_{1}\right\rangle,
\end{gathered}
$$

applying Remark 4.5 and Lemma 4.6, we obtain

$$
\begin{aligned}
\operatorname{Re}\left\langle\partial_{t} u, \partial_{t} \tilde{Q} u_{1}\right\rangle & =\operatorname{Re}\left\{\left\langle\partial_{t} \sigma u^{(0)}, \partial_{t} \tilde{Q} u_{1}\right\rangle+\left\langle\partial_{t} u_{1}, \partial_{t} \tilde{Q} u_{1}\right\rangle\right\} \\
& =\operatorname{Re}\left\{-\left\langle Q_{0} \tilde{B}_{\xi}\left(\sigma u^{(0)}+u_{1}\right)\right\rangle\left\langle u_{1}^{(0)}, \partial_{t} \tilde{Q} u_{1}\right\rangle+\left|\sqrt{\rho_{s}} \partial_{t} w_{1}\right|_{2}^{2}\right\} \\
& \geq \frac{7}{8}\left|\sqrt{\rho_{s}} \partial_{t} w_{1}\right|_{2}^{2}-C\left\{\frac{1}{\gamma^{4}}|\xi|^{2}\left(|\sigma|^{2}+\left|\phi_{1}\right|_{2}^{2}\right)+\frac{1}{v} \tilde{D}_{\xi}\left[w_{1}\right]\right\} .
\end{aligned}
$$

As for the second term on the left-hand side of (4.39), we see from $\widehat{L}_{0} u^{(0)}=0$ and $\widehat{B}_{0} u^{(0)}=0$ that

$$
\begin{aligned}
\left\langle\widehat{L}_{\xi} u, \partial_{t} \tilde{Q} u_{1}\right\rangle= & \left\langle\tilde{M}_{\xi}\left(\sigma u^{(0)}\right), \partial_{t} \tilde{Q} u_{1}\right\rangle+\left\langle\widehat{L}_{\xi} u_{1}, \partial_{t} \tilde{Q} u_{1}\right\rangle \\
= & \left\langle\tilde{A}_{\xi}\left(\sigma u^{(0)}\right), \partial_{t} \tilde{Q} u_{1}\right\rangle+\left\langle\widehat{B}_{\xi}\left(\sigma u^{(0)}+u_{1}\right), \partial_{t} \tilde{Q} u_{1}\right\rangle \\
& +\left\langle\widehat{A}_{\xi} u_{1}, \partial_{t} \tilde{Q} u_{1}\right\rangle+\left\langle\widehat{C}_{0} u_{1}, \partial_{t} \tilde{Q} u_{1}\right\rangle .
\end{aligned}
$$

It follows from (4.39)-(4.41) that

$$
\begin{aligned}
& \frac{7}{8}\left|\sqrt{\rho_{s}} \partial_{t} w_{1}\right|_{2}^{2}+\operatorname{Re}\left\langle\tilde{A}_{\xi}\left(\sigma u^{(0)}\right), \partial_{t} \tilde{Q} u_{1}\right\rangle+\operatorname{Re}\left\langle\widehat{B}_{\xi}\left(\sigma u^{(0)}+u_{1}\right), \partial_{t} \tilde{Q} u_{1}\right\rangle \\
& +\operatorname{Re}\left\langle\widehat{A}_{\xi} u_{1}, \partial_{t} \tilde{Q} u_{1}\right\rangle+\operatorname{Re}\left\langle\widehat{C}_{0} u_{1}, \partial_{t} \tilde{Q} u_{1}\right\rangle \\
& \quad \leq C\left\{\frac{1}{\gamma^{4}}|\xi|^{2}|\sigma|^{2}+\frac{1}{\gamma^{4}}|\xi|^{2}\left|\phi_{1}\right|_{2}^{2}+\frac{1}{v} \tilde{D}_{\xi}\left[w_{1}\right]\right\} .
\end{aligned}
$$

Next we show the estimate

$$
\begin{aligned}
& \operatorname{Re}\left\{\left\langle\widehat{B}_{\xi}\left(\sigma u^{(0)}+u_{1}\right), \partial_{t} \tilde{Q} u_{1}\right\rangle+\left\langle\widehat{A}_{\xi} u_{1}, \partial_{t} \tilde{Q} u_{1}\right\rangle\right\} \\
& \geq \frac{1}{2} \frac{d}{d t}\left(\tilde{D}_{\xi}\left[w_{1}\right]+J_{2}^{(0)}[u]\right)-\epsilon\left|\sqrt{\rho_{s}} \partial_{t} w_{1}\right|_{2}^{2} \\
&-C\left\{\left(\frac{1}{\gamma^{2}}+\frac{1}{\epsilon \gamma^{4}}\right)|\xi|^{2}|\sigma|^{2}+\frac{1}{\gamma^{2}}|\xi|^{2}\left|\phi_{1}\right|_{2}^{2}+\left(\frac{1}{v \gamma^{2}}+\frac{\gamma^{2}}{v}+\frac{1}{\epsilon v}\right) \tilde{D}_{\xi}\left[w_{1}\right]\right\}
\end{aligned}
$$

for any $\epsilon>0$ with $C$ independent of $\epsilon$. In fact, it holds by integrating by parts that

$$
\operatorname{Re}\left\langle\widehat{A}_{\xi} u_{1}, \partial_{t} \tilde{Q} u_{1}\right\rangle=\frac{1}{2} \frac{d}{d t} \tilde{D}_{\xi}\left[w_{1}\right] .
$$

Since $\widehat{B}_{\xi}^{*}=-\widehat{B}_{\xi}$, we see that

$$
\operatorname{Re}\left\langle\widehat{B}_{\xi}\left(\sigma u_{0}^{(0)}\right), \partial_{t} \tilde{Q} u_{1}\right\rangle=-\frac{d}{d t}\left\{\operatorname{Re}\left\langle\sigma u_{0}^{(0)}, \widehat{B}_{\xi} \tilde{Q} u_{1}\right\rangle\right\}+\operatorname{Re}\left\langle\partial_{t}\left(\sigma u_{0}^{(0)}\right), \widehat{B}_{\xi} \tilde{Q} u_{1}\right\rangle
$$


By (4.8) we have

$$
\partial_{t} \sigma=-\left\langle Q_{0} \tilde{B}_{\xi}\left(\sigma u^{(0)}+u_{1}\right)\right\rangle .
$$

It then follows from Lemma 4.6 that

$$
\begin{aligned}
\left|\operatorname{Re}\left\langle\partial_{t}\left(\sigma u_{0}^{(0)}\right), \widehat{B}_{\xi} \tilde{Q} u_{1}\right\rangle\right| & =\left|\operatorname{Re}\left\{\left\langle Q_{0} \tilde{B}_{\xi}\left(\sigma u^{(0)}+u_{1}\right)\right\rangle\left\langle u_{0}^{(0)}, \widehat{B}_{\xi} \tilde{Q} u_{1}\right\rangle\right\}\right| \\
& \leq C\left\{\frac{1}{\gamma^{2}}|\xi|^{2}\left(|\sigma|^{2}+\left|\phi_{1}\right|_{2}^{2}\right)+\left(\frac{1}{v \gamma^{2}}+\frac{\gamma^{2}}{v}\right) \tilde{D}_{\xi}\left[w_{1}\right]\right\} .
\end{aligned}
$$

Similarly to above, the following equation holds:

$$
\begin{aligned}
\operatorname{Re} & \left\langle\widehat{B}_{\xi} u_{1}, \partial_{t} \tilde{Q} u_{1}\right\rangle \\
& =-\frac{d}{d t}\left\{\operatorname{Re}\left\langle u_{1}, \widehat{B_{\xi}} \tilde{Q} u_{1}\right\rangle\right\}+\operatorname{Re}\left\langle\partial_{t} u_{1}, \widehat{B_{\xi}} \tilde{Q} u_{1}\right\rangle \\
& =-\frac{d}{d t}\left\{\operatorname{Re}\left\langle u_{1}, \widehat{B} \xi \tilde{Q} u_{1}\right\rangle\right\}+\operatorname{Re}\left\langle\partial_{t} Q_{0} u_{1}, \widehat{B} \xi \tilde{Q} u_{1}\right\rangle+\operatorname{Re}\left\langle\partial_{t} \tilde{Q} u_{1}, \widehat{B} \xi \tilde{Q} u_{1}\right\rangle .
\end{aligned}
$$

We estimate the second term on the right-hand side of (4.47). By (4.11) we have

$$
\begin{aligned}
\partial_{t} Q_{0} u_{1} & =-Q_{0}\left\{\widehat{L}_{\xi} u_{1}+\tilde{M}_{\xi}\left(\sigma u^{(0)}\right)-\left\langle Q_{0} \tilde{B}_{\xi}\left(\sigma u^{(0)}+u_{1}\right)\right\rangle u^{(0)}\right\} \\
& =-Q_{0} \widehat{B_{\xi}} u_{1}-Q_{0} \tilde{B}_{\xi}\left(\sigma u^{(0)}\right)+\left\langle Q_{0} \tilde{B}_{\xi}\left(\sigma u^{(0)}+u_{1}\right)\right\rangle u^{(0)} .
\end{aligned}
$$

Since $\left\langle\partial_{t} Q_{0} u_{1}, \widehat{B_{\xi}} \tilde{Q} u_{1}\right\rangle=\left\langle\partial_{t} Q_{0} u_{1}, Q_{0} \widehat{B_{\xi}} \tilde{Q} u_{1}\right\rangle$, we see from Lemma 4.6 that

$$
\begin{aligned}
& \left|\operatorname{Re}\left\langle\partial_{t} Q_{0} u_{1}, \widehat{B}_{\xi} \tilde{Q} u_{1}\right\rangle\right| \\
& \quad \leq C\left\{\left|Q_{0} \widehat{B}_{\xi} u_{1}\right|_{2}+\left|Q_{0} \tilde{B}_{\xi}\left(\sigma u^{(0)}\right)\right|_{2}+\left|\left\langle Q_{0} \tilde{B}_{\xi}\left(\sigma u^{(0)}+u_{1}\right)\right\rangle u^{(0)}\right|_{2}\right\} \times \frac{1}{\gamma^{2}}\left|Q_{0} \widehat{B}_{\xi} \tilde{Q} u_{1}\right|_{2} \\
& \quad \leq C\left\{\frac{1}{\gamma^{2}}|\xi|^{2}\left(|\sigma|^{2}+\left|\phi_{1}\right|_{2}^{2}\right)+\left(\frac{1}{v \gamma^{2}}+\frac{\gamma^{2}}{v}\right) \tilde{D}_{\xi}\left[w_{1}\right]\right\} .
\end{aligned}
$$

The third term on the right-hand side of (4.47) is estimated as

$$
\begin{aligned}
\left|\operatorname{Re}\left\langle\partial_{t} \tilde{Q} u_{1}, \widehat{B}_{\xi} \tilde{Q} u_{1}\right\rangle\right| & \leq C\left|\sqrt{\rho_{s}} \partial_{t} w_{1}\right|_{2}\left(\left|\nabla^{\prime} \cdot\left(\rho_{s} w_{1}^{\prime}\right)+i \xi \rho_{s} w_{1}^{3}\right|_{2}+|\xi|\left|w_{1}\right|_{2}\right) \\
& \leq \epsilon\left|\sqrt{\rho_{s}} \partial_{t} w_{1}\right|_{2}^{2}+C \frac{1}{\epsilon \nu} \tilde{D}_{\xi}\left[w_{1}\right]
\end{aligned}
$$

for any $\epsilon>0$ with $C$ independent of $\epsilon$. This, together with (4.47) and (4.48), leads to the inequality

$$
\begin{aligned}
& \operatorname{Re}\left\langle\widehat{B}_{\xi} u_{1}, \partial_{t} \tilde{Q} u_{1}\right\rangle \\
& \geq-\frac{d}{d t}\left\{\operatorname{Re}\left\langle u_{1}, \widehat{B}_{\xi} \tilde{Q} u_{1}\right\rangle\right\}-\epsilon\left|\sqrt{\rho_{s}} \partial_{t} w_{1}\right|_{2}^{2} \\
&-C\left\{\frac{1}{\gamma^{2}}|\xi|^{2}\left(|\sigma|^{2}+\left|\phi_{1}\right|_{2}^{2}\right)+\left(\frac{1}{v \gamma^{2}}+\frac{\gamma^{2}}{v}+\frac{1}{\epsilon v}\right) \tilde{D}_{\xi}\left[w_{1}\right]\right\}
\end{aligned}
$$

for any $\epsilon>0$ with $C$ independent of $\epsilon$. Furthermore, we have

$$
\begin{aligned}
\left|\operatorname{Re}\left\langle\widehat{B}_{\xi}\left(\sigma u_{1}^{(0)}\right), \partial_{t} \tilde{Q} u_{1}\right\rangle\right| & \leq C\left|\sqrt{\rho_{s}} \partial_{t} w_{1}\right|_{2}\left|i \xi \rho_{s} \sigma w^{(0), 3}+i \xi v_{s}^{3} \sigma w^{(0), 3}\right|_{2} \\
& \leq \epsilon\left|\sqrt{\rho_{s}} \partial_{t} w_{1}\right|_{2}^{2}+C \frac{1}{\epsilon \gamma^{4}}|\xi|^{2}|\sigma|^{2}
\end{aligned}
$$


for any $\epsilon>0$ with $C$ independent of $\epsilon$. By (4.45), (4.46), (4.49) and (4.50), we obtain

$$
\begin{aligned}
\operatorname{Re}\left\langle\widehat{B}_{\xi}\right. & \left.\left(\sigma u^{(0)}+u_{1}\right), \partial_{t} \tilde{Q} u_{1}\right\rangle \\
\geq & -\frac{1}{2} \frac{d}{d t} J_{2}^{(0)}[u]-\epsilon\left|\sqrt{\rho_{s}} \partial_{t} w_{1}\right|_{2}^{2} \\
& \quad-C\left\{\left(\frac{1}{\gamma^{2}}+\frac{1}{\epsilon \gamma^{4}}\right)|\xi|^{2}|\sigma|^{2}+\frac{1}{\gamma^{2}}|\xi|^{2}\left|\phi_{1}\right|_{2}^{2}+\left(\frac{1}{v \gamma^{2}}+\frac{\gamma^{2}}{v}+\frac{1}{\epsilon v}\right) \tilde{D}_{\xi}\left[w_{1}\right]\right\} .
\end{aligned}
$$

This, together with (4.44), gives (4.43).

The remaining terms on the left-hand side of (4.42) are estimated as

$$
\begin{aligned}
\left|\operatorname{Re}\left\langle\tilde{A}_{\xi}\left(\sigma u^{(0)}\right), \partial_{t} \tilde{Q} u_{1}\right\rangle\right| & \leq C\left\{\tilde{v}|\xi|\left|\nabla^{\prime} w^{(0), 3}\right|_{2}+(v+\tilde{v})|\xi|^{2}\left|w^{(0), 3}\right|{ }_{2}\right\}|\sigma|\left|\sqrt{\rho_{s}} \partial_{t} w_{1}\right|_{2} \\
& \leq \epsilon\left|\sqrt{\rho_{s}} \partial_{t} w_{1}\right|_{2}^{2}+C \frac{1}{\epsilon}\left\{\frac{\tilde{v}^{2}}{\gamma^{4}}|\xi|^{2}|\sigma|^{2}+\frac{(v+\tilde{v})^{2}}{\gamma^{4}}|\xi|^{4}|\sigma|^{2}\right\} \\
\left|\operatorname{Re}\left\langle\widehat{C}_{0} u_{1}, \partial_{t} \tilde{Q} u_{1}\right\rangle\right| & \leq C\left(\frac{v}{\gamma^{2}}\left|\phi_{1}\right|_{2}+\left|w_{1}^{\prime}\right|_{2}\right)\left|\sqrt{\rho_{s}} \partial_{t} w_{1}\right|_{2} \\
& \leq \epsilon\left|\sqrt{\rho_{s}} \partial_{t} w_{1}\right|_{2}^{2}+C \frac{1}{\epsilon}\left\{\frac{v^{2}}{\gamma^{4}}\left|\phi_{1}\right|_{2}^{2}+\frac{1}{v} \tilde{D}_{\xi}\left[w_{1}\right]\right\} .
\end{aligned}
$$

Here $\epsilon$ is an arbitrary positive number and $C$ is a constant independent of $\epsilon$. Taking $\epsilon>0$ suitably small, we see from (4.42) with (4.43), (4.51) and (4.52) that if $v \geq 1$ and $\gamma^{2} \geq 1$, then

$$
\begin{aligned}
\frac{1}{2} \frac{d}{d t}\left(\tilde{D}_{\xi}\left[w_{1}\right]+J_{2}^{(0)}[u]\right)+\frac{3}{4}\left|\sqrt{\rho_{s}} \partial_{t} w_{1}\right|_{2}^{2} \\
\leq C_{0}\left\{\left(\frac{1}{\gamma^{2}}+\frac{\tilde{v}^{2}}{\gamma^{4}}\right)|\xi|^{2}|\sigma|^{2}+\frac{(\nu+\tilde{v})^{2}}{\gamma^{4}}|\xi|^{4}|\sigma|^{2}+\frac{v^{2}}{\gamma^{4}}\left|\phi_{1}\right|_{2}^{2}\right. \\
\left.\quad+\frac{1}{\gamma^{2}}|\xi|^{2}\left|\phi_{1}\right|_{2}^{2}+\frac{\gamma^{2}}{v} \tilde{D}_{\xi}\left[w_{1}\right]\right\} .
\end{aligned}
$$

We take $b_{3}$ as $b_{3} \geq \max \left\{b_{3}^{*}, 4 C_{0}\right\}$ and then add (4.53) to $b_{3}\left(\gamma^{2} / v\right) \times(4.12)$, to obtain (4.38). This completes the proof.

We next establish the estimate for higher-order derivatives near the boundary $\partial D$. We introduce the local curvilinear coordinate system.

For any $\bar{x}_{0}^{\prime} \in \partial D$, there exist a neighborhood $\tilde{\mathcal{O}}_{\bar{x}_{0}}$ of $\bar{x}_{0}^{\prime}$ and a smooth diffeomorphism map $\Psi=\left(\Psi_{1}, \Psi_{2}\right): \tilde{\mathcal{O}}_{\bar{x}_{0}^{\prime}} \rightarrow B_{1}(0)=\left\{z^{\prime}=\left(z_{1}, z_{2}\right):\left|z^{\prime}\right|<1\right\}$ such that

$$
\left\{\begin{array}{l}
\Psi\left(\tilde{\mathcal{O}}_{\bar{x}_{0}^{\prime}} \cap D\right)=\left\{z^{\prime} \in B_{1}(0): z_{1}>0\right\} \\
\Psi\left(\tilde{\mathcal{O}}_{\bar{x}_{0}^{\prime}} \cap \partial D\right)=\left\{z^{\prime} \in B_{1}(0): z_{1}=0\right\} \\
\operatorname{det} \nabla_{x^{\prime}} \Psi \neq 0 \text { on } \quad \tilde{\mathcal{O}}_{\bar{x}_{0}^{\prime}} \cap D
\end{array}\right.
$$

By the tubular neighborhood theorem, there exist a neighborhood $\mathcal{O}_{\bar{x}_{0}^{\prime}}$ of $\bar{x}_{0}^{\prime}$ and a local curvilinear coordinate system $y^{\prime}=\left(y_{1}, y_{2}\right)$ on $\mathcal{O}_{\bar{x}_{0}^{\prime}}$ defined by

$$
x^{\prime}=y_{1} a_{1}\left(y_{2}\right)+\Psi^{-1}\left(0, y_{2}\right): \mathcal{R} \rightarrow \mathcal{O}_{\bar{x}_{0}^{\prime}},
$$


where $\mathcal{R}=\left\{y^{\prime}=\left(y_{1}, y_{2}\right):\left|y_{1}\right| \leq \tilde{\delta}_{1},\left|y_{2}\right| \leq \tilde{\delta}_{2}\right\}$ for some $\tilde{\delta}_{1}, \tilde{\delta}_{2}>0 ; a_{1}\left(y_{2}\right)$ is the unit inward normal to $\partial D$ that is given by

$$
a_{1}\left(y_{2}\right)=\frac{\nabla_{x^{\prime}} \Psi_{1}}{\left|\nabla_{x^{\prime}} \Psi_{1}\right|} .
$$

We set $y_{3}=x_{3}$. It then follows that

$$
\begin{gathered}
\nabla_{x}=e_{1}\left(y_{2}\right) \partial_{y_{1}}+J\left(y^{\prime}\right) e_{2}\left(y_{2}\right) \partial_{y_{2}}+e_{3} \partial_{y_{3}}, \\
\nabla_{y}=\left(\begin{array}{c}
{ }^{\mathrm{T}} e_{1}\left(y_{2}\right) \\
\frac{1}{J\left(y^{\prime}\right)}{ }^{\mathrm{T}} e_{2}\left(y_{2}\right) \\
\mathrm{T}_{e_{3}}
\end{array}\right) \nabla_{x},
\end{gathered}
$$

where

$$
\begin{gathered}
e_{1}\left(y_{2}\right)=\left(\begin{array}{c}
a_{1}\left(y_{2}\right) \\
0
\end{array}\right), \quad e_{2}\left(y_{2}\right)=\left(\begin{array}{c}
a_{2}\left(y_{2}\right) \\
0
\end{array}\right), \quad e_{3}=\left(\begin{array}{l}
0 \\
0 \\
1
\end{array}\right) ; \\
J\left(y^{\prime}\right)=\left|\operatorname{det} \nabla_{x^{\prime}} \Psi\right|, \quad a_{2}\left(y_{2}\right)=\frac{-\nabla_{x^{\prime}}^{\perp} \Psi_{1}}{\left|\nabla_{x^{\prime}}^{\perp} \Psi_{1}\right|}
\end{gathered}
$$

with $\nabla_{x^{\prime}}^{\perp} \Psi_{1}={ }^{\mathrm{T}}\left(-\partial_{x_{2}} \Psi_{1}, \partial_{x_{1}} \Psi_{1}\right)$. Note that $\partial_{y_{1}}$ and $\partial_{y_{2}}$ are the inward normal derivative and tangential derivative at $x^{\prime}=\Psi^{-1}\left(0, y_{2}\right) \in \partial D \cap \mathcal{O}_{\bar{x}_{0}^{\prime}}$, respectively. We denote the normal and tangential derivatives by $\partial_{n}$ and $\partial$, i.e.

$$
\partial_{n}=\partial_{y_{1}}, \quad \partial=\partial_{y_{2}} .
$$

Since $\partial D$ is compact, there are bounded open sets $\mathcal{O}_{m}(m=1, \ldots, N)$ such that $\partial D \subset$ $\bigcup_{m=1}^{N} \mathcal{O}_{m}$ and for each $m=1, \ldots, N$, there exists a local curvilinear coordinate system $y^{\prime}=\left(y_{1}, y_{2}\right)$ as defined in (4.54) with $\mathcal{O}_{\bar{x}_{0}^{\prime}}, \Psi$ and $\mathcal{R}$ replaced by $\mathcal{O}_{m}, \Psi^{m}$ and $\mathcal{R}_{m}=\left\{y^{\prime}=\right.$ $\left.\left(y_{1}, y_{2}\right):\left|y_{1}\right|<\tilde{\delta}_{1}^{m},\left|y_{2}\right|<\tilde{\delta}_{2}^{m}\right\}$ for some $\tilde{\delta}_{1}^{m}, \tilde{\delta}_{2}^{m}>0$. At last, we take an open set $\mathcal{O}_{0} \subset D$ such that

$$
\bigcup_{m=0}^{N} \mathcal{O}_{m} \supset D, \quad \overline{\mathcal{O}}_{0} \cap \partial D=\emptyset .
$$

We set a local coordinate $y^{\prime}=\left(y_{1}, y_{2}\right)$ such that $y_{1}=x_{1}, y_{2}=x_{2}$ on $\mathcal{O}_{0}$.

Note that if $h \in H^{2}(D)$, then $\left.h\right|_{\partial D}=0$ implies that $\left.\partial^{k} h\right|_{\partial D \cap \mathcal{O}^{m}}=0(k=0,1)$.

Let us introduce a partition of unity $\left\{\chi_{m}\right\}_{m=0}^{N}$ subordinate to $\left\{\mathcal{O}_{m}\right\}_{m=0}^{N}$, satisfying

$$
\sum_{m=0}^{N} \chi_{m}=1 \quad \text { on } D, \quad \chi_{m} \in C_{0}^{\infty}\left(\mathcal{O}_{m}\right) \quad(m=0,1, \ldots, N) .
$$

In the following we will denote by $[A, B]$ the commutator of $A$ and $B$, i.e.

$$
[A, B]=A B-B A .
$$

LEMMA 4.14. For $1 \leq m \leq N$ the following estimates hold:

(i) $\quad\left|\left[\partial, \partial_{x_{j}}\right] h\right| \leq C\left|\partial_{x^{\prime}} h\right|$ for $h \in H^{2}(D)$ and $j=1,2$; 
(ii) $\left|\left(\chi_{m}\left[\partial, \partial_{x_{j}}\right] h, \chi_{m} \partial h\right)\right| \leq C\left|\chi_{m} \partial_{x^{\prime}} h\right|_{2}^{2}$ for $h \in H^{2}(D)$ and $j=1,2$;

(iii) $\left|\left(\chi_{m}\left[\partial, \partial_{x_{k}} \partial_{x_{l}}\right] h, \chi_{m} \partial h\right)\right| \leq \eta\left|\chi_{m} \partial_{x^{\prime}} \partial h\right|_{2}^{2}+C(1+1 / \eta)\left|\partial_{x^{\prime}} h\right|_{L^{2}\left(D \cap \mathcal{O}_{m}\right)}^{2}$ for all $\eta>0$, $h \in H^{2}(D)$ with $\left.\partial h\right|_{\partial D \cap \mathcal{O}_{m}}=0$ and $k, l=1,2$.

Proof. (i) For $x^{\prime} \in D \cap \mathcal{O}_{m}$, we set $y^{\prime}=\Psi^{m}\left(x^{\prime}\right), h\left(x^{\prime}\right)=\tilde{h}\left(y^{\prime}\right)$. Then there exists a smooth matrix valued function $A_{1}\left(y^{\prime}\right)$ such that $\nabla_{x^{\prime}}=A_{1}\left(y^{\prime}\right) \nabla_{y^{\prime}}$. We thus find that

$$
\left[\partial, \partial_{x_{j}}\right] h=\partial \partial_{x_{j}} h-\partial_{x_{j}} \partial h=\sum_{0 \leq l_{1}, 0 \leq l_{2}, l_{1}+l_{2}=1} h_{l_{1} l_{2}} \partial^{l_{1}} \partial_{n}^{l_{2}} \tilde{h},
$$

where $h_{l_{1} l_{2}}=h_{l_{1} l_{2}}\left(y^{\prime}\right)$ are smooth functions depending only on $D \cap \mathcal{O}_{m}$. Since

$$
\frac{1}{C}\left|\partial_{y^{\prime}} \tilde{h}\right| \leq\left|\partial_{x^{\prime}} h\right| \leq C\left|\partial_{y^{\prime}} \tilde{h}\right|
$$

for some constant $C>0$, we have the desired inequality. This completes the proof of (i).

(ii) The estimate in (ii) immediately follows from (i).

(iii) We have $\nabla_{y^{\prime}}=A_{1}\left(y^{\prime}\right)^{-1} \nabla_{x^{\prime}}$. We set $A_{1}\left(y^{\prime}\right)^{-1}=\left(c^{i j}\left(x^{\prime}\right)\right)_{i j}$. Then it holds that

$$
\left[\partial, \partial_{x_{k} x_{l}}\right] h=-\sum_{j=1}^{2}\left\{\partial_{x_{k}} \partial_{x_{l}} c^{2 j} \partial_{x_{j}} h+\partial_{x_{l}} c^{2 j} \partial_{x_{k}} \partial_{x_{j}} h+\partial_{x_{k}} c^{2 j} \partial_{x_{l}} \partial_{x_{k}} h\right\} .
$$

It follows from integration by parts that

$$
\begin{aligned}
& \left|\left(\chi_{m} \partial_{x_{l}} c^{2 j} \partial_{x_{k}} \partial_{x_{j}} h, \chi_{m} \partial h\right)\right| \\
& \quad=\left|\left(\chi_{m} \partial_{x_{l}} c^{2 j} \partial_{x_{j}} h, \chi_{m} \partial_{x_{k}} \partial h\right)+\left(\chi_{m} \partial_{x_{k}} \partial_{x_{l}} c^{2 j} \partial_{x_{j}} h, \chi_{m} \partial h\right)+\left(\partial_{x_{k}} \chi_{m}^{2} \partial_{x_{l}} c^{2 j} \partial_{x_{j}} h, \partial h\right)\right| \\
& \quad \leq C\left\{\left|\chi_{m} \partial_{x_{j}} h\right|_{2}\left|\chi_{m} \partial_{x_{k}} \partial h\right|_{2}+\left|\chi_{m} \partial_{x_{j}} h\right|_{2}\left|\chi_{m} \partial h\right|_{2}+\left|\partial_{x_{j}} h\right|_{L^{2}\left(D \cap \mathcal{O}_{m}\right)}\left|\chi_{m} \partial h\right|_{2}\right\} \\
& \quad \leq \eta\left|\chi_{m} \partial_{x^{\prime}} \partial h\right|_{2}^{2}+C\left(1+\frac{1}{\eta}\right)\left|\partial_{x^{\prime}} h\right|_{L^{2}\left(D \cap \mathcal{O}_{m}\right)}^{2} .
\end{aligned}
$$

This completes the proof of (iii).

We are in a position to estimate higher-order derivatives. We first derive the estimate for $\partial \phi_{1}$.

Proposition 4.15. For $1 \leq m \leq N$, there exist constants $v_{0}>0, \omega_{0}>0$ and $b>0$ such that if $v \geq v_{0}, \gamma^{2} \geq 1$ and $(v+\tilde{v}) \omega / v \leq \omega_{0}$, then the following estimate holds:

$$
\begin{aligned}
\frac{1}{2} \frac{d}{d t}( & \left.\frac{1}{\gamma^{2}}\left|\chi_{m} \sqrt{\frac{P^{\prime}\left(\rho_{s}\right)}{\gamma^{2} \rho_{s}}} \partial \phi_{1}\right|_{2}^{2}+\left|\chi_{m} \sqrt{\rho_{s}} \partial w_{1}\right|_{2}^{2}\right)+b \frac{v+\tilde{v}}{\gamma^{4}}\left|\chi_{m} \partial \dot{\phi}_{1}\right|_{2}^{2} \\
+ & \frac{1}{2} \nu\left(\left|\chi_{m} \nabla^{\prime} \partial w_{1}\right|_{2}^{2}+|\xi|^{2}\left|\chi_{m} \partial w_{1}\right|_{2}^{2}\right)+\frac{1}{2} \tilde{v}\left|\chi_{m}\left(\nabla^{\prime} \cdot \partial w_{1}^{\prime}+i \xi \partial w_{1}^{3}\right)\right|_{2}^{2} \\
\leq & C\left\{\left(\frac{1}{\gamma^{2}}+\frac{v+\tilde{v}}{\gamma^{4}}\right)|\xi|^{2}|\sigma|^{2}+\left(\eta+\frac{1}{\gamma^{2}}\right)\left|\phi_{1}\right|_{2}^{2}+\left(\eta+\frac{1}{\gamma^{2}}+\frac{v+\tilde{v}}{\gamma^{4}}\right)|\xi|^{2}\left|\phi_{1}\right|_{2}^{2}\right. \\
& \left.+\left(\eta+\frac{1}{\gamma^{2}}\right)\left|\partial_{x^{\prime}} \phi_{1}\right|_{2}^{2}+\left(\frac{1}{\eta v}+\frac{v}{\gamma^{2}}+\frac{\tilde{v}}{v}+1\right) \tilde{D}_{\xi}\left[w_{1}\right]+\left(\frac{\tilde{v}}{v}+1\right)|\xi|^{2} \tilde{D}_{\xi}\left[w_{1}\right]\right\}
\end{aligned}
$$

for any $\eta>0$ with $C$ independent of $\eta$. 
Proof. Applying d to (4.11), we have

$$
\left\{\begin{array}{l}
\partial_{t} \partial \phi_{1}+i \xi v_{s}^{3} \partial \phi_{1}+\gamma^{2} \nabla^{\prime} \cdot\left(\rho_{s} \partial w_{1}^{\prime}\right)+\gamma^{2} i \xi \rho_{s} \partial w_{1}^{3}=\tilde{F}^{0}, \\
\partial_{t} \partial w_{1}^{\prime}-\frac{v}{\rho_{s}}\left(\Delta^{\prime}-|\xi|^{2}\right) \partial w_{1}^{\prime}-\frac{\tilde{v}}{\rho_{s}} \nabla^{\prime}\left(\nabla^{\prime} \cdot \partial w_{1}^{\prime}+i \xi \partial w_{1}^{3}\right) \\
+\nabla^{\prime}\left(\frac{P^{\prime}\left(\rho_{s}\right)}{\gamma^{2} \rho_{s}} \partial \phi_{1}\right)+i \xi v_{s}^{3} \partial w_{1}^{\prime}=\tilde{G}^{\prime}, \\
\partial_{t} \partial w_{1}^{3}-\frac{v}{\rho_{s}}\left(\Delta^{\prime}-|\xi|^{2}\right) \partial w_{1}^{3}-\frac{\tilde{v}}{\rho_{s}} i \xi\left(\nabla^{\prime} \cdot \partial w_{1}^{\prime}+i \xi \partial w_{1}^{3}\right) \\
\quad+i \xi \frac{P^{\prime}\left(\rho_{s}\right)}{\gamma^{2} \rho_{s}} \partial \phi_{1}+i \xi v_{s}^{3} \partial w_{1}^{3}+\frac{v}{\gamma^{2} \rho_{s}^{2}} \Delta^{\prime} v_{s}^{3} \partial \phi_{1}+\partial w_{1}^{\prime} \cdot \nabla^{\prime} v_{s}^{3}=\tilde{G}^{3}
\end{array}\right.
$$

on $D \cap \mathcal{O}_{m}$ and

$$
\left.\partial w_{1}\right|_{\partial D \cap \mathcal{O}_{m}}=0
$$

Here $\tilde{F}^{0}=F_{1}^{0}+F_{2}^{0}, \tilde{G}^{\prime}=G_{1}^{\prime}+G_{2}^{\prime}$ and $\tilde{G}^{3}=G_{1}^{3}+G_{2}^{3}$, with

$$
\begin{aligned}
F_{1}^{0}= & -\left[\partial, i \xi v_{s}^{3}\right] \phi_{1}-\gamma^{2}\left[\partial, \nabla^{\prime} \cdot \rho_{s}\right] w_{1}^{\prime}-\gamma^{2}\left[\partial, i \xi \rho_{s}\right] w_{1}^{3}, \\
G_{1}^{\prime}= & \nu\left[\partial, \frac{1}{\rho_{s}} \Delta^{\prime}\right] w_{1}^{\prime}-v\left[\partial, \frac{1}{\rho_{s}}|\xi|^{2}\right] w_{1}^{\prime}+\tilde{v}\left[\partial, \frac{1}{\rho_{s}} \nabla^{\prime} \nabla^{\prime} \cdot\right] w_{1}^{\prime}+\tilde{v}\left[\partial, \frac{1}{\rho_{s}} \nabla^{\prime}(i \xi)\right] w_{1}^{3} \\
& -\left[\partial, \nabla^{\prime} \frac{P^{\prime}\left(\rho_{s}\right)}{\gamma^{2} \rho_{s}}\right] \phi_{1}-\left[\partial, i \xi v_{s}^{3}\right] w_{1}^{\prime}, \\
G_{1}^{3}= & v\left[\partial, \frac{1}{\rho_{s}} \Delta^{\prime}\right] w_{1}^{3}-v\left[\partial, \frac{1}{\rho_{s}}|\xi|^{2}\right] w_{1}^{3}+\tilde{v}\left[\partial, \frac{1}{\rho_{s}} i \xi \nabla^{\prime} \cdot\right] w_{1}^{\prime}-\tilde{v}\left[\partial, \frac{1}{\rho_{s}}|\xi|^{2}\right] w_{1}^{3} \\
& -\left[\partial, \frac{v}{\gamma^{2} \rho_{s}^{2}} \Delta^{\prime} v_{s}^{3}\right] \phi_{1}-\left[\partial, i \xi \frac{P^{\prime}\left(\rho_{s}\right)}{\gamma^{2} \rho_{s}}\right] \phi_{1}-\left[\partial, i \xi v_{s}^{3}\right] w_{1}^{3}-\left[\partial,{ }^{\mathrm{T}}\left(\nabla^{\prime} v_{s}^{3}\right)\right] w_{1}^{\prime}, \\
F_{2}^{0}= & -\left\{i \xi \sigma \partial\left(v_{s}^{3} \phi^{(0)}\right)+\gamma^{2} i \xi \sigma \partial\left(\rho_{s} w^{(0), 3}\right)-\left\langle Q_{0} \tilde{B}_{\xi}\left(\sigma u^{(0)}+u_{1}\right)\right\rangle \partial \phi^{(0)}\right\}, \\
G_{2}^{\prime}= & -\left\{-\tilde{v} i \xi \sigma \partial\left(\frac{1}{\rho_{s}} \nabla^{\prime} w^{(0), 3}\right)\right\}, \\
G_{2}^{3}= & -\left\{(v+\tilde{v}) \xi^{2} \sigma \partial\left(\frac{1}{\rho_{s}} w^{(0), 3}\right)+i \xi \sigma \partial\left(v_{s}^{3} w^{(0), 3}\right)-\left\langle Q_{0} \tilde{B}_{\xi}\left(\sigma u^{(0)}+u_{1}\right)\right\rangle \partial w^{(0), 3}\right\} .
\end{aligned}
$$

We set $\tilde{F}={ }^{\mathrm{T}}\left(\tilde{F}^{0}, \tilde{G}^{\prime}, \tilde{G}^{3}\right), F_{1}={ }^{\mathrm{T}}\left(F_{1}^{0}, G_{1}^{\prime}, G_{1}^{3}\right)$ and $F_{2}={ }^{\mathrm{T}}\left(F_{2}^{0}, G_{2}^{\prime}, G_{2}^{3}\right)$. Taking the weighted inner product of (4.57) with $\chi_{m}^{2} \partial u_{1}$, we have

$$
\begin{aligned}
\frac{1}{2} \frac{d}{d t} & \left(\frac{1}{\gamma^{2}}\left|\chi_{m} \sqrt{\frac{P^{\prime}\left(\rho_{s}\right)}{\gamma^{2} \rho_{s}}} \partial \phi_{1}\right|_{2}^{2}+\left|\chi_{m} \sqrt{\rho_{s}} \partial w_{1}\right|_{2}^{2}\right) \\
+ & v\left\{\left|\chi_{m} \nabla^{\prime} \partial w_{1}\right|_{2}^{2}+|\xi|^{2}\left|\chi_{m} \partial w_{1}\right|_{2}^{2}\right\}+\tilde{v}\left|\chi_{m}\left(\nabla^{\prime} \cdot \partial w_{1}^{\prime}+i \xi \partial w_{1}^{3}\right)\right|_{2}^{2} \\
\quad & =\operatorname{Re}\left\{\left\langle F, \chi_{m}^{2} \partial u_{1}\right\rangle-I\right\},
\end{aligned}
$$


where

$$
\begin{aligned}
I= & v\left(\nabla^{\prime} \partial w_{1}, \nabla^{\prime}\left(\chi_{m}^{2}\right) \partial w_{1}\right)+\tilde{v}\left(\nabla^{\prime} \cdot \partial w_{1}^{\prime}+i \xi \partial w_{1}^{3}, \nabla^{\prime}\left(\chi_{m}^{2}\right) \cdot \partial w_{1}^{\prime}\right) \\
& -\left(\frac{P^{\prime}\left(\rho_{s}\right)}{\gamma^{2} \rho_{s}} \partial \phi_{1}, \nabla^{\prime}\left(\chi_{m}^{2}\right) \cdot \rho_{s} \partial w_{1}^{\prime}\right)+\left(i \xi v_{s}^{3} \partial w_{1}, \chi_{m}^{2} \rho_{s} \partial w_{1}\right) \\
& +\left(\frac{v}{\gamma^{2} \rho_{s}} \Delta^{\prime} v_{s}^{3} \partial \phi_{1}, \chi_{m}^{2} \partial w_{1}^{3}\right)+\left(\partial w_{1}^{\prime} \cdot \nabla^{\prime} v_{s}^{3}, \chi_{m}^{2} \rho_{s} \partial w_{1}^{3}\right) .
\end{aligned}
$$

Let us estimate the right-hand side of (4.58). By Lemma 4.14 and the Poincaré inequality we have

$$
\begin{aligned}
\mid \operatorname{Re}\langle & \left.F_{1}, \chi_{m}^{2} \partial u_{1}\right\rangle \mid \\
\leq & \left(\eta+\frac{C}{\gamma^{2}}\right)\left|\phi_{1}\right|_{2}^{2}+\left(\eta+\frac{C}{\gamma^{2}}\right)|\xi|^{2}\left|\phi_{1}\right|_{2}^{2}+\left(\eta+\frac{C}{\gamma^{2}}\right)\left|\partial_{x^{\prime}} \phi_{1}\right|_{2}^{2} \\
& +C\left(\frac{1}{v \eta}+\frac{v}{\gamma^{2}}+\frac{\tilde{v}}{v}+\frac{1}{v}+1\right) \tilde{D}_{\xi}\left[w_{1}\right]+\frac{1}{8} v\left(\left|\chi_{m} \nabla^{\prime} \partial w_{1}\right|_{2}^{2}+|\xi|^{2}\left|\chi_{m} \partial w_{1}\right|_{2}^{2}\right) \\
& +\frac{1}{8} \tilde{v}\left|\chi_{m}\left(\nabla^{\prime} \cdot \partial w_{1}^{\prime}+i \xi \partial w_{1}^{3}\right)\right|_{2}^{2}, \\
|\operatorname{Re} I| & \leq\left(\eta+\frac{C}{\gamma^{2}}\right)\left|\partial_{x^{\prime}} \phi_{1}\right|_{2}^{2}+C\left(\frac{v}{\gamma^{2}}+\frac{1}{v \eta}+\frac{1}{v}+1\right) \tilde{D}_{\xi}\left[w_{1}\right] \\
& +\frac{1}{8} v\left(\left|\chi_{m} \nabla^{\prime} \partial w_{1}\right|_{2}^{2}+|\xi|^{2}\left|\chi_{m} \partial w_{1}\right|_{2}^{2}\right)+\frac{1}{8} \tilde{v}\left|\chi_{m}\left(\nabla^{\prime} \cdot \partial w_{1}^{\prime}+i \xi \partial w_{1}^{3}\right)\right|_{2}^{2}
\end{aligned}
$$

for any $\eta>0$ with $C$ independent of $\eta>0$. By Lemma 4.6 and the Hölder inequality we deduce that

$$
\begin{aligned}
\mid \operatorname{Re}\langle & \left.F_{2}, \chi_{m}^{2} \partial u_{1}\right\rangle \mid \\
\leq & C\left\{\left(\frac{1}{\gamma^{2}}+\frac{v+\tilde{v}}{\gamma^{4}}\right)|\xi|^{2}|\sigma|^{2}+\left(\frac{1}{\gamma^{2}}+\frac{1}{\gamma^{4}}\right)|\xi|^{2}\left|\phi_{1}\right|_{2}^{2}+\left(\eta+\frac{1}{\gamma^{2}}\right)\left|\partial_{x^{\prime}} \phi_{1}\right|_{2}^{2}\right. \\
& \left.+\left(\frac{1}{v}+\frac{1}{v \eta}\right) \tilde{D}_{\xi}\left[w_{1}\right]+\left(\frac{\tilde{v}}{v}+1\right)|\xi|^{2} \tilde{D}_{\xi}\left[w_{1}\right]\right\}
\end{aligned}
$$

for any $\eta>0$ with $C$ independent of $\eta>0$. Therefore, we see from (4.58) that if $v \geq 1$, $\gamma^{2} \geq 1$ and $\omega \leq 1$, then

$$
\begin{aligned}
& \frac{1}{2} \frac{d}{d t}\left(\frac{1}{\gamma^{2}}\left|\chi_{m} \sqrt{\frac{P^{\prime}\left(\rho_{s}\right)}{\gamma^{2} \rho_{s}}} \partial \phi_{1}\right|_{2}^{2}+\left|\chi_{m} \sqrt{\rho_{s}} \partial w_{1}\right|_{2}^{2}\right) \\
& +\frac{3}{4} v\left(\left|\chi_{m} \nabla^{\prime} \partial w_{1}\right|_{2}^{2}+|\xi|^{2}\left|\chi_{m} \partial w_{1}\right|_{2}^{2}\right)+\frac{3}{4} \tilde{v}\left|\chi_{m}\left(\nabla^{\prime} \cdot \partial w_{1}^{\prime}+i \xi \partial w_{1}^{3}\right)\right|_{2}^{2} \\
& \quad \leq C\left\{\left(\frac{1}{\gamma^{2}}+\frac{v+\tilde{v}}{\gamma^{4}}\right)|\xi|^{2}|\sigma|^{2}+\left(\eta+\frac{1}{\gamma^{2}}\right)\left|\phi_{1}\right|_{2}^{2}+\left(\eta+\frac{1}{\gamma^{2}}+\frac{1}{\gamma^{4}}\right)|\xi|^{2}\left|\phi_{1}\right|_{2}^{2}\right. \\
& \left.\quad+\left(\eta+\frac{1}{\gamma^{2}}\right)\left|\partial_{x^{\prime}} \phi_{1}\right|_{2}^{2}+\left(\frac{1}{v \eta}+\frac{v}{\gamma^{2}}+\frac{\tilde{v}}{v}+1\right) \tilde{D}_{\xi}\left[w_{1}\right]+\left(\frac{\tilde{v}}{v}+1\right)|\xi|^{2} \tilde{D}_{\xi}\left[w_{1}\right]\right\} .
\end{aligned}
$$


We next estimate $\partial \dot{\phi}_{1}$. The first equation of (4.57) leads to

$$
\begin{aligned}
\frac{1}{\gamma^{2}} \partial \dot{\phi}_{1} & =\frac{1}{\gamma^{2}}\left(\partial_{t} \partial \phi_{1}+i \xi \partial\left(v_{s}^{3} \phi_{1}\right)\right) \\
& =\frac{1}{\gamma^{2}} \tilde{F}^{0}-\left\{\frac{1}{\gamma^{2}} i \xi \partial v_{s}^{3} \phi_{1}+\nabla^{\prime} \cdot\left(\rho_{s} \partial w_{1}^{\prime}\right)+i \xi \rho_{s} \partial w_{1}^{3}\right\}
\end{aligned}
$$

We thus have

$$
\frac{1}{\gamma^{4}}\left|\chi_{m} \partial \dot{\phi}_{1}\right|_{2}^{2} \leq C\left\{\frac{1}{\gamma^{4}}|\xi|^{2}|\sigma|^{2}+\frac{1}{\gamma^{4}}|\xi|^{2}\left|\phi_{1}\right|^{2}+\frac{1}{v} \tilde{D}_{\xi}\left[w_{1}\right]+\left|\chi_{m}\left(\nabla^{\prime} \cdot \partial w_{1}^{\prime}+i \xi \partial w_{1}^{3}\right)\right|_{2}^{2}\right\}
$$

Take $b>0$ suitably small and add $b\left((\nu+\tilde{v}) / \gamma^{4}\right)\left|\chi_{m} \partial \dot{\phi}_{1}\right|_{2}^{2}$ to (4.59). We thus obtain the desired estimate. This completes the proof.

We next derive the estimate for $\partial_{n} \phi_{1}$.

Proposition 4.16. For $1 \leq m \leq N$, there exist constants $\nu_{0}>0, \omega_{0}>0$ and $b>0$ such that if $v \geq v_{0}, \gamma^{2} \geq 1$ and $(v+\tilde{v}) \omega / v \leq \omega_{0}$, then the following estimate holds:

$$
\begin{aligned}
\frac{1}{2} \frac{d}{d t}( & \left.\frac{1}{\gamma^{2}}\left|\chi_{m} \sqrt{\frac{P^{\prime}\left(\rho_{s}\right)}{\gamma^{2} \rho_{s}}} \partial_{n} \phi_{1}\right|_{2}^{2}\right)+\frac{1}{2} \frac{1}{v+\tilde{v}}\left|\chi_{m} \frac{P^{\prime}\left(\rho_{s}\right)}{\gamma^{2}} \partial_{n} \phi_{1}\right|_{2}^{2}+b \frac{v+\tilde{v}}{\gamma^{4}}\left|\chi_{m} \partial_{n} \dot{\phi}_{1}\right|_{2}^{2} \\
\leq & C\left\{\frac{v+\tilde{v}}{\gamma^{4}}|\xi|^{2}|\sigma|^{2}+\left(\frac{\omega^{2}}{v+\tilde{v}}+\frac{v^{2}}{\gamma^{4}(v+\tilde{v})}\right)\left|\phi_{1}\right|_{2}^{2}+\frac{v+\tilde{v}}{\gamma^{4}}|\xi|^{2}\left|\phi_{1}\right|_{2}^{2}\right. \\
& +\left(\frac{\tilde{v}}{v}+1\right) \tilde{D}_{\xi}\left[w_{1}\right]+\frac{v}{v+\tilde{v}}|\xi|^{2} \tilde{D}_{\xi}\left[w_{1}\right]+\frac{v^{2}}{v+\tilde{v}}\left(\left|\chi_{m} \partial_{n} \partial w_{1}\right|_{2}^{2}+\left|\chi_{m} \partial^{2} w_{1}\right|_{2}^{2}\right) \\
& \left.+\frac{1}{v+\tilde{v}}\left|\sqrt{\rho_{s}} \partial_{t} w_{1}\right|_{2}^{2}\right\} .
\end{aligned}
$$

Proof. For a scalar field $p\left(x^{\prime}\right)$ on $D \cap \mathcal{O}_{m}$, we set

$$
\tilde{p}\left(y^{\prime}\right)=p\left(x^{\prime}\right) \quad\left(y^{\prime}=\Psi^{m}\left(x^{\prime}\right), x^{\prime} \in D \cap \mathcal{O}_{m}\right) .
$$

Similarly we transform a vector field $h\left(x^{\prime}\right)={ }^{\mathrm{T}}\left(h^{1}\left(x^{\prime}\right), h^{2}\left(x^{\prime}\right), h^{3}\left(x^{\prime}\right)\right)$ into $\tilde{h}\left(y^{\prime}\right)=$ $\mathrm{T}\left(\tilde{h}^{1}\left(y^{\prime}\right), \tilde{h}^{2}\left(y^{\prime}\right), \tilde{h}^{3}\left(y^{\prime}\right)\right)$ as

$$
h\left(x^{\prime}\right)=E\left(y^{\prime}\right) \tilde{h}\left(y^{\prime}\right),
$$

where $E\left(y^{\prime}\right)=\left(e_{1}\left(y_{2}^{\prime}\right), e_{2}\left(y_{2}\right), e_{3}\right)$ with $e_{1}\left(y_{2}\right), e_{2}\left(y_{2}\right)$ and $e_{3}$ given in (4.55). Note that, since $e_{3}={ }^{\mathrm{T}}(0,0,1)$, the Fourier transform in $x_{3}=y_{3}$ commutes with these transformations. It then follows that $\tilde{\phi}_{1}\left(y^{\prime}\right)$ and $\tilde{w}_{1}\left(y^{\prime}\right)={ }^{\mathrm{T}}\left(\tilde{w}_{1}^{1}\left(y^{\prime}\right), \tilde{w}_{1}^{2}\left(y^{\prime}\right), \tilde{w}_{1}^{3}\left(y^{\prime}\right)\right)$ are governed by the following 
system of equations

$$
\left\{\begin{array}{l}
\partial_{t} \tilde{\phi}_{1}+i \xi \tilde{v}_{s}^{3} \tilde{\phi}_{1}+\gamma^{2} \widehat{\operatorname{div}}_{y}\left(\tilde{\rho}_{s} \tilde{w}_{1}\right)+i \xi \tilde{v}_{s}^{3} \sigma \tilde{\phi}^{(0)}+\gamma^{2} i \xi \tilde{\rho}_{s} \sigma \tilde{w}^{(0), 3} \\
\quad-\left\langle Q_{0} \tilde{B}_{\xi}\left(\sigma \tilde{u}^{(0)}+\tilde{u}_{1}\right)\right\rangle \tilde{\phi}^{(0)}=0 \\
\partial_{t} \tilde{w}_{1}^{1}+\frac{v}{\tilde{\rho}_{s}}\left(\widehat{\operatorname{rot}}_{y} \widehat{\operatorname{rot}}_{y} \tilde{w}_{1}\right)^{1}-\frac{v+\tilde{v}}{\tilde{\rho}_{s}}\left(\widehat{\nabla}_{y} \widehat{\operatorname{div}}_{y} \tilde{w}_{1}\right)^{1}+\partial_{y_{1}}\left(\frac{\tilde{P}^{\prime}\left(\tilde{\rho}_{s}\right)}{\gamma^{2} \tilde{\rho}_{s}} \tilde{\phi}_{1}\right) \\
\quad+\frac{v}{\gamma^{2} \tilde{\rho}_{s}^{2}}\left(\Delta_{y^{\prime}} \tilde{v}_{s}\right)^{1} \tilde{\phi}_{1}+i \xi \tilde{v}_{s}^{3} \tilde{w}_{1}^{1}-\frac{\tilde{v}}{\tilde{\rho}_{s}} i \xi \sigma \partial_{y_{1}} \tilde{w}^{(0), 3}=0 \\
\partial_{t} \tilde{w}_{1}^{2}+\frac{v}{\tilde{\rho}_{s}}\left(\widehat{\operatorname{rot}}_{y} \widehat{\operatorname{rot}}_{y} \tilde{w}_{1}\right)^{2}-\frac{v+\tilde{v}}{\tilde{\rho}_{s}}\left(\widehat{\nabla}_{y} \widehat{\operatorname{div}}_{y} \tilde{w}_{1}\right)^{2}+\frac{1}{J} \partial_{y_{2}}\left(\frac{\tilde{P}^{\prime}\left(\tilde{\rho}_{s}\right)}{\gamma^{2} \tilde{\rho}_{s}} \tilde{\phi}_{1}\right) \\
\quad+\frac{v}{\gamma^{2} \tilde{\rho}_{s}^{2}}\left(\Delta_{y^{\prime}} \tilde{v}_{s}\right)^{2} \tilde{\phi}_{1}+i \xi \tilde{v}_{s}^{3} \tilde{w}_{1}^{2}-\frac{\tilde{v}}{\tilde{\rho}_{s}} i \xi \sigma \frac{1}{J} \partial_{y_{2}} \tilde{w}^{(0), 3}=0 \\
\quad+\frac{v}{\partial_{t}} \tilde{w}_{1}^{3}+\frac{v}{\tilde{\rho}_{s}}\left(\widehat{\operatorname{rot}}_{y} \widehat{\operatorname{rot}}_{y} \tilde{w}_{1}\right)^{3}-\frac{v+\tilde{\rho}_{s}^{2}}{\tilde{\rho}_{s}}\left(\widehat{\nabla}_{y} \widehat{\operatorname{div}}_{y} \tilde{w}_{1}\right)^{3}+i \xi \frac{\tilde{P}^{\prime}\left(\tilde{\rho}_{s}\right)}{\gamma^{2} \tilde{\rho}_{s}} \tilde{\phi}_{1}+i \xi \tilde{v}_{s}^{3} \tilde{w}_{1}^{3}+\tilde{w}_{1}^{1} \partial_{y_{1}} \tilde{v}_{s}^{3}+\frac{1}{J} \tilde{w}_{1}^{2} \partial_{y_{2}} \tilde{v}_{s}^{3}+\frac{v+\tilde{v}_{\rho_{s}}}{\rho^{2} \sigma \tilde{w}^{(0), 3}} \\
\quad+i \xi \frac{\tilde{P}^{\prime}\left(\tilde{\rho}_{s}\right)}{\gamma^{2} \tilde{\rho}_{s}} \sigma \tilde{\phi}^{(0)}+i \xi \tilde{v}_{s}^{3} \sigma \tilde{w}^{(0), 3}+\left\langle Q_{0} \tilde{B}_{\xi}\left(\sigma \tilde{u}^{(0)}+\tilde{u}_{1}\right)\right\rangle \tilde{w}^{(0), 3}=0
\end{array}\right.
$$

with $\tilde{\rho}_{s}\left(y^{\prime}\right)=\rho_{s}\left(x^{\prime}\right), \tilde{v}_{s}^{3}\left(y^{\prime}\right)=v_{s}^{3}\left(x^{\prime}\right)$ and $\tilde{P}^{\prime}\left(\tilde{\rho}_{s}\left(y^{\prime}\right)\right)=P^{\prime}\left(\rho_{s}\left(x^{\prime}\right)\right)$. Here $\nabla_{y}, \operatorname{div}_{y}$ and $\operatorname{rot}_{y}$ denote the gradient, divergence and rotation in the curvilinear coordinate $y$ which are written for $\tilde{p}=\tilde{p}\left(y^{\prime}\right)$ and $\tilde{h}={ }^{\mathrm{T}}\left(\tilde{h}^{1}\left(y^{\prime}\right), \tilde{h}^{2}\left(y^{\prime}\right), \tilde{h}^{3}\left(y^{\prime}\right)\right)$ as

$$
\begin{aligned}
\nabla_{y} \tilde{p} & =e_{1} \partial_{y_{1}} \tilde{p}+\frac{1}{J} e_{2} \partial_{y_{2}} \tilde{p}+e_{3} \partial_{y_{3}} \tilde{p}, \\
\operatorname{div}_{y} \tilde{h} & =\frac{1}{J}\left\{\partial_{y_{1}}\left(J \tilde{h}^{1}\right)+\partial_{y_{2}} \tilde{h}^{2}+\partial_{y_{3}}\left(J \tilde{h}^{3}\right)\right\}, \\
\operatorname{rot}_{y} \tilde{h} & =\left(\operatorname{rot}_{y} \tilde{h}\right)^{1} e_{1}+\left(\operatorname{rot}_{y} \tilde{h}\right)^{2} e_{2}+\left(\operatorname{rot}_{y} \tilde{h}\right)^{3} e_{3}
\end{aligned}
$$

with

$$
\begin{aligned}
& \left(\operatorname{rot}_{y} \tilde{h}\right)^{1}=\frac{1}{J}\left\{\partial_{y_{2}} \tilde{h}^{3}-\partial_{y_{3}}\left(J \tilde{h}^{2}\right)\right\}, \\
& \left(\operatorname{rot}_{y} \tilde{h}\right)^{2}=\partial_{y_{3}} \tilde{h}^{1}-\partial_{y_{1}} \tilde{h}^{3}, \\
& \left(\operatorname{rot}_{y} \tilde{h}\right)^{3}=\frac{1}{J}\left\{\partial_{y_{1}} \tilde{h}^{2}-\partial_{y_{2}}\left(J \tilde{h}^{1}\right)\right\},
\end{aligned}
$$

and, therefore,

$$
\begin{aligned}
& \left(\operatorname{rot}_{y} \operatorname{rot}_{y} \tilde{h}\right)^{1}=\frac{1}{J}\left\{\partial_{y_{2}}\left(\operatorname{rot}_{y} \tilde{h}\right)^{3}-\partial_{y_{3}}\left(\operatorname{rot}_{y} \tilde{h}\right)^{2}\right\}, \\
& \left(\operatorname{rot}_{y} \operatorname{rot}_{y} \tilde{h}\right)^{2}=\partial_{y_{3}}\left(\operatorname{rot}_{y} \tilde{h}\right)^{1}-\partial_{y_{1}}\left(\operatorname{rot}_{y} \tilde{h}\right)^{3}, \\
& \left(\operatorname{rot}_{y} \operatorname{rot}_{y} \tilde{h}\right)^{3}=\frac{1}{J}\left\{\partial_{y_{1}}\left(\operatorname{rot}_{y} \tilde{h}\right)^{2}-\partial_{y_{2}}\left(\operatorname{rot}_{y} \tilde{h}\right)^{1}\right\} ;
\end{aligned}
$$

the Fourier transformed gradient $\widehat{\nabla}_{y}$ is given by

$$
\widehat{\nabla}_{y} \tilde{p}=e_{1} \partial_{y_{1}} \tilde{p}+\frac{1}{J} e_{2} \partial_{y_{2}} \tilde{p}+e_{3} i \xi \tilde{p} ;
$$


and similarly $\widehat{\operatorname{div}}_{y}$ and $\widehat{\operatorname{rot}}_{y}$ are obtained from $\operatorname{div}_{y}$ and $\operatorname{rot}_{y}$ by replacing $\partial_{y_{3}}$ with $i \xi$ respectively. Applying $\partial_{y_{1}}$ to the first equation of (4.61), we have

$$
\begin{aligned}
\partial_{t} \partial_{y_{1}} & \tilde{\phi}_{1}+i \xi \tilde{v}_{s}^{3} \partial_{y_{1}} \tilde{\phi}_{1}+\gamma^{2} \tilde{\rho}_{s} \partial_{y_{1}} \widehat{\operatorname{div}}_{y} \tilde{w}_{1} \\
= & -\left\{i \xi \partial_{y_{1}} \tilde{v}_{s}^{3} \tilde{\phi}_{1}+\gamma^{2} \partial_{y_{1}}\left(\widehat{\operatorname{div}}_{y}\left(\tilde{\rho}_{s} \tilde{w}_{1}\right)\right)-\gamma^{2} \tilde{\rho}_{s} \partial_{y_{1}} \widehat{\operatorname{div}}_{y} \tilde{w}_{1}\right. \\
& \left.+i \xi \partial_{y_{1}}\left(\tilde{v}_{s}^{3} \sigma \tilde{\phi}^{(0)}\right)+\gamma^{2} i \xi \partial_{y_{1}}\left(\tilde{\rho}_{s} \sigma \tilde{w}^{(0), 3}\right)-\left\langle Q_{0} \tilde{B}_{\xi}\left(\sigma \tilde{u}^{(0)}+\tilde{u}_{1}\right)\right\rangle \partial_{y_{1}} \tilde{\phi}^{(0)}\right\} .
\end{aligned}
$$

To eliminate the term $\partial_{y_{1}} \partial_{y_{1}} \tilde{w}_{1}^{1}$ in this equation, we consider $\gamma^{2} \tilde{\rho}_{s} /(v+\tilde{v}) \times(4.61)_{2}+$ $1 / \tilde{\rho}_{s} \times(4.62)$. It then follows that

$$
\frac{1}{\tilde{\rho}_{s}} \partial_{t} \partial_{y_{1}} \tilde{\phi}_{1}+\frac{\tilde{P}^{\prime}\left(\tilde{\rho}_{s}\right)}{v+\tilde{v}} \partial_{y_{1}} \tilde{\phi}_{1}+\frac{1}{\tilde{\rho}_{s}} i \xi \tilde{v}_{s}^{3} \partial_{y_{1}} \tilde{\phi}_{1}=I,
$$

where $I=I_{1}+I_{2}$ with

$$
\begin{aligned}
I_{1}= & -\frac{\gamma^{2}}{v+\tilde{v}}\left\{\tilde{\rho}_{s} \partial_{t} \tilde{w}_{1}^{1}+v\left(\widehat{\operatorname{rot}}_{y} \widehat{\operatorname{rot}}_{y} \tilde{w}_{1}\right)^{1}\right. \\
& \left.+\tilde{\rho}_{s} \partial_{y_{1}}\left(\frac{\tilde{P}^{\prime}\left(\tilde{\rho}_{s}\right)}{\gamma^{2} \tilde{\rho}_{s}}\right) \tilde{\phi}_{1}+\frac{v}{\gamma^{2}} \tilde{\rho}_{s}\left(\Delta_{y^{\prime}} \tilde{v}_{s}\right)^{1} \tilde{\phi}_{1}+i \xi \tilde{\rho}_{s} \tilde{v}_{s}^{3} \tilde{w}_{1}^{1}\right\} \\
& -\left\{i \xi \frac{1}{\tilde{\rho}_{s}} \partial_{y_{1}} \tilde{v}_{s}^{3} \tilde{\phi}_{1}+\gamma^{2} \frac{1}{\tilde{\rho}_{s}} \partial_{y_{1}}\left(\widehat{\operatorname{div}}_{y}\left(\tilde{\rho}_{s} \tilde{w}_{1}\right)\right)-\gamma^{2} \partial_{y_{1}} \widehat{\operatorname{div}}_{y} \tilde{w}_{1}\right\}, \\
I_{2}= & -\frac{\gamma^{2}}{v+\tilde{v}}\left(-\tilde{v} i \xi \sigma \partial_{y_{1}} \tilde{w}^{(0), 3}\right)-\left\{i \xi \frac{1}{\tilde{\rho}_{s}} \partial_{y_{1}}\left(\tilde{v}_{s}^{3} \sigma \tilde{\phi}^{(0)}\right)\right. \\
& \left.+\gamma^{2} i \xi \frac{1}{\tilde{\rho}_{s}} \partial_{y_{1}}\left(\tilde{\rho}_{s} \sigma \tilde{w}^{(0), 3}\right)-\frac{1}{\tilde{\rho}_{s}}\left\langle Q_{0} \tilde{B}_{\xi}\left(\sigma \tilde{u}^{(0)}+\tilde{u}_{1}\right)\right\rangle \partial_{y_{1}} \tilde{\phi}^{(0)}\right\} .
\end{aligned}
$$

Considering $\int_{\Psi^{m}\left(D \cap \mathcal{O}_{m}\right)}(4.63) \times \tilde{\chi}_{m}^{2}\left(\tilde{P}^{\prime}\left(\tilde{\rho}_{s}\right) / \gamma^{4}\right) \overline{\partial_{y_{1}} \tilde{\phi}_{1}} J d y^{\prime}$ with $\tilde{\chi}_{m}\left(y^{\prime}\right)=\chi_{m}\left(x^{\prime}\right)$, we see that

$$
\begin{aligned}
& \frac{1}{2} \frac{d}{d t}\left(\frac{1}{\gamma^{2}}\left|\tilde{\chi}_{m} \sqrt{\frac{\tilde{P}^{\prime}\left(\tilde{\rho}_{s}\right)}{\gamma^{2} \tilde{\rho}_{s}}} \partial_{y_{1}} \tilde{\phi}_{1}\right|_{2}^{2}\right)+\frac{1}{v+\tilde{v}}\left|\tilde{\chi}_{m} \frac{\tilde{P}^{\prime}\left(\tilde{\rho}_{s}\right)}{\gamma^{2}} \partial_{y_{1}} \tilde{\phi}_{1}\right|_{2}^{2} \\
& =\int_{\Psi^{m}\left(D \cap \mathcal{O}_{m}\right)} I \times \tilde{\chi}_{m}^{2} \frac{\tilde{P}^{\prime}\left(\tilde{\rho}_{s}\right)}{\gamma^{4}} \overline{\partial_{y_{1}} \tilde{\phi}_{1}} J d y^{\prime} .
\end{aligned}
$$

Since

$$
\left.\widehat{\operatorname{rot}}_{y} \widehat{\operatorname{rot}}_{y} \tilde{w}_{1}\right)^{1}=\frac{1}{J} \partial_{y_{2}}\left(\frac{1}{J} \partial_{y_{1}}\left(J \tilde{w}_{1}^{2}\right)-\frac{1}{J} \partial_{y_{2}} \tilde{w}_{1}^{1}\right)-i \xi\left(i \xi \tilde{w}_{1}^{1}-\partial_{y_{1}} \tilde{w}_{1}^{3}\right)
$$


we obtain

$$
\begin{aligned}
& \frac{v+\tilde{v}}{\gamma^{4}}\left|\tilde{\chi}_{m} I_{1}\right|_{2}^{2} \\
& \leq C\left\{\left(\frac{\omega^{2}}{v+\tilde{v}}+\frac{v^{2}}{\gamma^{4}(v+\tilde{v})}\right)\left|\tilde{\chi}_{m} \tilde{\phi}_{1}\right|_{2}^{2}+\frac{v+\tilde{v}}{\gamma^{4}}|\xi|^{2}\left|\tilde{\chi}_{m} \tilde{\phi}_{1}\right|_{2}^{2}+\frac{1}{v+\tilde{v}}\left|\tilde{\chi}_{m} \sqrt{\tilde{\rho}_{s}} \partial_{t} \tilde{w}_{1}\right|_{2}^{2}\right. \\
& \quad+(v+\tilde{v}) \omega^{2}\left|\tilde{\chi}_{m} \tilde{w}_{1}\right|_{2}^{2}+\frac{1}{v+\tilde{v}}|\xi|^{2}\left|\tilde{\chi}_{m} \tilde{w}_{1}\right|_{2}^{2}+\frac{v^{2}}{v+\tilde{v}}|\xi|^{4}\left|\tilde{\chi}_{m} \tilde{w}_{1}\right|_{2}^{2} \\
& \left.\quad+(v+\tilde{v}) \omega^{2}\left|\tilde{\chi}_{m} \partial_{y^{\prime}} \tilde{w}_{1}\right|_{2}^{2}+\frac{v^{2}}{v+\tilde{v}^{2}}|\xi|^{2}\left|\tilde{\chi}_{m} \partial_{y^{\prime}} \tilde{w}_{1}\right|_{2}^{2}+\frac{v^{2}}{v+\tilde{v}}\left|\tilde{\chi}_{m} \partial_{y^{\prime}} \partial_{y_{2}} \tilde{w}_{1}\right|_{2}^{2}\right\} \\
& \frac{v+\tilde{v}}{\gamma^{4}}\left|\tilde{\chi}_{m} I_{2}\right|_{2}^{2} \\
& \leq C\left\{\frac{v+\tilde{v}}{\left.\gamma^{4}|\xi|^{2}|\sigma|^{2}+\frac{v+\tilde{v}}{\gamma^{4}}|\xi|^{2}\left|\tilde{\phi}_{1}\right|_{L^{2}\left(\Psi^{m}\left(D \cap \mathcal{O}_{m}\right)\right)}^{2}+(v+\tilde{v})\left|\tilde{w}_{1}\right|_{L^{2}\left(\Psi^{m}\left(D \cap \mathcal{O}_{m}\right)\right)}^{2}\right\} .}\right.
\end{aligned}
$$

It then follows that

$$
\begin{aligned}
\frac{1}{2} \frac{d}{d t}( & \left.\frac{1}{\gamma^{2}}\left|\tilde{\chi}_{m} \sqrt{\frac{\tilde{P}^{\prime}\left(\tilde{\rho}_{s}\right)}{\gamma^{2} \tilde{\rho}_{s}}} \partial_{y_{1}} \tilde{\phi}_{1}\right|_{2}^{2}\right)+\frac{3}{4} \frac{1}{v+\tilde{v}}\left|\tilde{\chi}_{m} \frac{\tilde{P}^{\prime}\left(\tilde{\rho}_{s}\right)}{\gamma^{2}} \partial_{y_{1}} \tilde{\phi}_{1}\right|_{2}^{2} \\
\leq & C\left\{\frac{v+\tilde{v}}{\gamma^{4}}|\xi|^{2}|\sigma|^{2}+\left(\frac{\omega^{2}}{v+\tilde{v}}+\frac{v^{2}}{\gamma^{4}(v+\tilde{v})}\right)\left|\tilde{\chi}_{m} \tilde{\phi}_{1}\right|_{2}^{2}+\frac{v+\tilde{v}}{\gamma^{4}}|\xi|^{2}\left|\tilde{\phi}_{1}\right|_{L^{2}\left(\Psi^{m}\left(D \cap \mathcal{O}_{m}\right)\right)}^{2}\right. \\
& +(v+\tilde{v})\left|\tilde{w}_{1}\right|_{L^{2}\left(\Psi^{m}\left(D \cap \mathcal{O}_{m}\right)\right)}^{2}+\frac{1}{v+\tilde{v}}|\xi|^{2}\left|\tilde{\chi}_{m} \tilde{w}_{1}\right|_{2}^{2}+\frac{v^{2}}{v+\tilde{v}}|\xi|^{4}\left|\tilde{\chi}_{m} \tilde{w}_{1}\right|_{2}^{2} \\
& +(v+\tilde{v}) \omega^{2}\left|\tilde{\chi}_{m} \partial_{y^{\prime}} \tilde{w}_{1}\right|_{2}^{2}+\frac{v^{2}}{v+\tilde{v}}|\xi|^{2}\left|\tilde{\chi}_{m} \partial_{y^{\prime}} \tilde{w}_{1}\right|_{2}^{2}+\frac{v^{2}}{v+\tilde{v}}\left|\tilde{\chi}_{m} \partial_{y^{\prime}} \partial_{y_{2}} \tilde{w}_{1}\right|_{2}^{2} \\
& \left.+\frac{1}{v+\tilde{v}}\left|\tilde{\chi}_{m} \sqrt{\tilde{\rho}_{s}} \partial_{t} \tilde{w}_{1}\right|_{2}^{2}\right\}
\end{aligned}
$$

We next consider $\partial_{y_{1}} \dot{\tilde{\phi}}_{1}$ where $\dot{\tilde{\phi}}_{1}=\partial_{t} \tilde{\phi}_{1}+i \xi \tilde{v}_{s}^{3} \tilde{\phi}_{1}$. Equation (4.63) gives that

$$
\frac{1}{\gamma^{2}} \partial_{y_{1}} \dot{\tilde{\phi}}_{1}=\frac{1}{\gamma^{2} \tilde{\rho}_{s}}\left(I+i \xi \partial_{y_{1}} \tilde{v}_{s}^{3} \tilde{\phi}_{1}-\frac{\gamma^{2}}{v+\tilde{v}} \frac{\tilde{P}^{\prime}\left(\tilde{\rho}_{s}\right)}{\gamma^{2} \tilde{\rho}_{s}} \partial_{y_{1}} \tilde{\phi}_{1}\right) .
$$

This equation leads to the estimate

$$
\begin{aligned}
\frac{v+\tilde{v}}{\gamma^{4}}\left|\tilde{\chi}_{m} \partial_{y_{1}} \dot{\tilde{\phi}}_{1}\right|_{2}^{2} & \leq C\left\{\frac{v+\tilde{v}}{\gamma^{4}}\left|\tilde{\chi}_{m} \tilde{\rho}_{s}\left(I+i \xi \partial_{y_{1}} \tilde{v}_{s}^{3} \tilde{\phi}_{1}\right)\right|_{2}^{2}+\frac{1}{v+\tilde{v}}\left|\tilde{\chi}_{m} \frac{\tilde{P}^{\prime}\left(\tilde{\rho}_{s}\right)}{\gamma^{2}} \partial_{y_{1}} \tilde{\phi}_{1}\right|_{2}^{2}\right\} \\
& \leq C\left\{\frac{v+\tilde{v}}{\gamma^{4}}\left|\tilde{\chi}_{m} I\right|_{2}^{2}+\frac{1}{v+\tilde{v}}\left|\tilde{\chi}_{m} \frac{\tilde{P}^{\prime}\left(\tilde{\rho}_{s}\right)}{\gamma^{2}} \partial_{y_{1}} \tilde{\phi}_{1}\right|_{2}^{2}\right\} .
\end{aligned}
$$


Therefore, if we take $b>0$ suitably small and add $b\left((v+\tilde{v}) / \gamma^{4}\right)\left|\tilde{\chi}_{m} \partial_{y_{1}} \dot{\tilde{\phi}}_{1}\right|_{2}^{2}$ to (4.64), we obtain

$$
\begin{aligned}
\frac{1}{2} \frac{d}{d t}( & \left.\frac{1}{\gamma^{2}}\left|\tilde{\chi}_{m} \sqrt{\frac{\tilde{P}^{\prime}\left(\tilde{\rho}_{s}\right)}{\gamma^{2} \tilde{\rho}_{s}}} \partial_{y_{1}} \tilde{\phi}_{1}\right|_{2}^{2}\right)+\frac{1}{2} \frac{1}{v+\tilde{v}}\left|\tilde{\chi}_{m} \frac{\tilde{P}^{\prime}\left(\tilde{\rho}_{s}\right)}{\gamma^{2}} \partial_{y_{1}} \tilde{\phi}_{1}\right|_{2}^{2}+b \frac{v+\tilde{v}}{\gamma^{4}}\left|\tilde{\chi}_{m} \partial_{y_{1}} \dot{\tilde{\phi}}_{1}\right|_{2}^{2} \\
\leq & C\left\{\frac{v+\tilde{v}}{\gamma^{4}}|\xi|^{2}|\sigma|^{2}+\left(\frac{\omega^{2}}{v+\tilde{v}}+\frac{v^{2}}{\gamma^{4}(v+\tilde{v})}\right)\left|\tilde{\chi}_{m} \tilde{\phi}_{1}\right|_{2}^{2}+\frac{v+\tilde{v}}{\gamma^{4}}|\xi|^{2}\left|\tilde{\phi}_{1}\right|_{L^{2}\left(\Psi^{m}\left(D \cap \mathcal{O}_{m}\right)\right)}^{2}\right. \\
& +(v+\tilde{v})\left|\tilde{w}_{1}\right|_{L^{2}\left(\Psi^{m}\left(D \cap \mathcal{O}_{m}\right)\right)}^{2}+\frac{1}{v+\tilde{v}}|\xi|^{2}\left|\tilde{\chi}_{m} \tilde{w}_{1}\right|_{2}^{2}+\frac{v^{2}}{v+\tilde{v}}|\xi|^{4}\left|\tilde{\chi}_{m} \tilde{w}_{1}\right|_{2}^{2} \\
& +(v+\tilde{v}) \omega^{2}\left|\tilde{\chi}_{m} \partial_{y^{\prime}} \tilde{w}_{1}\right|_{2}^{2}+\frac{v^{2}}{v+\tilde{v}}|\xi|^{2}\left|\tilde{\chi}_{m} \partial_{y^{\prime}} \tilde{w}_{1}\right|_{2}^{2}+\frac{v^{2}}{v+\tilde{v}}\left|\tilde{\chi}_{m} \partial_{y^{\prime}} \partial_{y_{2}} \tilde{w}_{1}\right|_{2}^{2} \\
& \left.+\frac{1}{v+\tilde{v}}\left|\tilde{\chi}_{m} \sqrt{\tilde{\rho}_{s}} \partial_{t} \tilde{w}_{1}\right|_{2}^{2}\right\} .
\end{aligned}
$$

The desired estimate follows from (4.65) by inverting to the original coordinates $x^{\prime}$ and noting that $\partial_{y_{1}}=\partial_{n}, \partial_{y_{2}}=\partial$. This completes the proof.

We next derive the interior estimate for the derivative of $\phi_{1}$.

Proposition 4.17. There exist constants $v_{0}>0, \omega_{0}>0$ and $b>0$ such that if $v \geq v_{0}$, $\gamma^{2} \geq 1$ and $(v+\tilde{v}) \omega / v \leq \omega_{0}$, then the following estimate holds:

$$
\begin{aligned}
\frac{1}{2} \frac{d}{d t}( & \left.\frac{1}{\gamma^{2}}\left|\chi_{0} \sqrt{\frac{P^{\prime}\left(\rho_{s}\right)}{\gamma^{2} \rho_{s}}} \partial_{x^{\prime}} \phi_{1}\right|_{2}^{2}+\left|\chi_{0} \sqrt{\rho_{s}} \partial_{x^{\prime}} w_{1}\right|_{2}^{2}\right)+b \frac{v+\tilde{v}}{\gamma^{4}}\left|\chi_{0} \partial_{x^{\prime}} \dot{\phi}_{1}\right|_{2}^{2} \\
+ & \frac{1}{2} v\left(\left|\chi_{0} \nabla^{\prime} \partial_{x^{\prime}} w_{1}\right|_{2}^{2}+|\xi|^{2}\left|\chi_{0} \partial_{x^{\prime}} w_{1}\right|_{2}^{2}\right)+\frac{1}{2} \tilde{v}\left|\chi_{0}\left(\nabla^{\prime} \cdot \partial_{x^{\prime}} w_{1}^{\prime}+i \xi \partial_{x^{\prime}} w_{1}^{3}\right)\right|_{2}^{2} \\
\leq & C\left\{\left(\frac{1}{\gamma^{2}}+\frac{v+\tilde{v}}{\gamma^{4}}\right)|\xi|^{2}|\sigma|^{2}+\frac{1}{\gamma^{2}}\left|\phi_{1}\right|_{2}^{2}+\left(\frac{1}{\gamma^{2}}+\frac{v+\tilde{v}}{\gamma^{4}}+\frac{\omega^{2}}{v+\tilde{v}}\right)|\xi|^{2}\left|\phi_{1}\right|_{2}^{2}\right. \\
& \left.+\left(\eta+\frac{1}{\gamma^{2}}\right)\left|\partial_{x^{\prime}} \phi_{1}\right|_{2}^{2}+\left(\frac{1}{\eta v}+\frac{v}{\gamma^{2}}+\frac{\tilde{v}}{v}+1\right) \tilde{D}_{\xi}\left[w_{1}\right]+\left(\frac{\tilde{v}}{v}+1\right)|\xi|^{2} \tilde{D}_{\xi}\left[w_{1}\right]\right\}
\end{aligned}
$$

for any $\eta>0$ with $C$ independent of $\eta$.

Since $\operatorname{supp}\left(\chi_{0} w_{1}\right) \subset D$ we have $\left.\partial_{x^{\prime}} w_{1}\right|_{\partial D \cap \mathcal{O}_{0}}=0$. Therefore we can prove this proposition similarly to the proof of Proposition 4.15. We omit the details.

Before proceeding further we introduce an energy functional. We define $E_{3}^{(0)}\left[u_{1}\right]$ by

$$
\begin{aligned}
E_{3}^{(0)}\left[u_{1}\right] & \\
= & \frac{1}{\gamma^{2}}\left|\chi_{0} \sqrt{\frac{P^{\prime}\left(\rho_{s}\right)}{\gamma^{2} \rho_{s}}} \partial_{x^{\prime}} \phi_{1}\right|_{2}^{2}+\left|\chi_{0} \sqrt{\rho_{s}} \partial_{x^{\prime}} w_{1}\right|_{2}^{2} \\
& +b_{4} \sum_{m=1}^{N}\left(\frac{1}{\gamma^{2}}\left|\chi_{m} \sqrt{\frac{P^{\prime}\left(\rho_{s}\right)}{\gamma^{2} \rho_{s}}} \partial \phi_{1}\right|_{2}^{2}+\left|\chi_{m} \sqrt{\rho_{s}} \partial w_{1}\right|_{2}^{2}\right)+\sum_{m=1}^{N} \frac{1}{\gamma^{2}}\left|\chi_{m} \sqrt{\frac{P^{\prime}\left(\rho_{s}\right)}{\gamma^{2} \rho_{s}}} \partial_{n} \phi_{1}\right|_{2}^{2},
\end{aligned}
$$

where $b_{4}$ is a positive constant. Taking $b_{4}$ suitably large, we have the following estimate for $E_{3}^{(0)}\left[u_{1}\right]$. 
PROPOSITION 4.18. There exist constants $v_{0}>0, \omega_{0}>0, b>0$ and $b_{4}>0$ such that if $v \geq v_{0}, \gamma^{2} \geq 1$ and $(v+\tilde{v}) \omega / v \leq \omega_{0}$, then the following estimate holds:

$$
\begin{aligned}
& \frac{1}{2} \frac{d}{d t} E_{3}^{(0)}\left[u_{1}\right]+b \frac{v+\tilde{v}}{\gamma^{4}}\left|\partial_{x^{\prime}} \dot{\phi}_{1}\right|_{2}^{2} \\
&+\frac{1}{2}\left\{v\left(\left|\chi_{0} \nabla^{\prime} \partial_{x^{\prime}} w_{1}\right|_{2}^{2}+|\xi|^{2}\left|\chi_{0} \partial_{x^{\prime}} w_{1}\right|_{2}^{2}\right)+\tilde{v}\left|\chi_{0}\left(\nabla^{\prime} \cdot \partial_{x^{\prime}} w_{1}^{\prime}+i \xi \partial_{x^{\prime}} w_{1}^{3}\right)\right|_{2}^{2}\right\} \\
&+\frac{1}{2} \sum_{m=1}^{N}\left\{v\left(\left|\chi_{m} \nabla^{\prime} \partial w_{1}\right|_{2}^{2}+|\xi|^{2}\left|\chi_{m} \partial w_{1}\right|_{2}^{2}\right)+\tilde{v}\left|\chi_{m}\left(\nabla^{\prime} \cdot \partial w_{1}^{\prime}+i \xi \partial w_{1}^{3}\right)\right|_{2}^{2}\right\} \\
& \leq C\left\{\left(\frac{1}{\gamma^{2}}+\frac{v+\tilde{v}}{\gamma^{4}}\right)|\xi|^{2}|\sigma|^{2}+\left(\eta+\frac{\omega^{2}}{v+\tilde{v}}+\frac{1}{\gamma^{2}}+\frac{v^{2}}{\gamma^{4}(v+\tilde{v})}\right)\left|\phi_{1}\right|_{2}^{2}\right. \\
&+\left(\eta+\frac{\omega^{2}}{v+\tilde{v}}+\frac{1}{\gamma^{2}}+\frac{v+\tilde{v}}{\gamma^{4}}\right)|\xi|^{2}\left|\phi_{1}\right|_{2}^{2}+\left(\eta+\frac{1}{\gamma^{2}}\right)\left|\partial_{x^{\prime}} \phi_{1}\right|_{2}^{2} \\
&\left.+\left(\frac{1}{v \eta}+\frac{v}{\gamma^{2}}+\frac{\tilde{v}}{v}+1\right) \tilde{D}_{\xi}\left[w_{1}\right]+\left(\frac{\tilde{v}}{v}+1\right)|\xi|^{2} \tilde{D}_{\xi}\left[w_{1}\right]+\frac{1}{v+\tilde{v}}\left|\sqrt{\rho_{s}} \partial_{t} w_{1}\right|_{2}^{2}\right\}
\end{aligned}
$$

for any $\eta>0$ with $C$ independent of $\eta$.

Using Propositions 4.15, 4.16 and 4.17, we obtain the estimate of Proposition 4.18.

We next derive a dissipative estimate for $\left|\partial_{x^{\prime}}^{2} w_{1}\right|_{2}$ and $\left|\partial_{x^{\prime}} \phi_{1}\right|_{2}$.

Proposition 4.19. There exist constants $v_{0}>0$ and $\omega_{0}>0$ such that if $v \geq v_{0}$, $(v+\tilde{v}) \omega / v \leq \omega_{0}$ and $\gamma^{2} \geq 1$, then the following estimate holds:

$$
\begin{aligned}
& \frac{v^{2}}{v+\tilde{v}}\left|\partial_{x}^{2} w_{1}^{\prime}\right|_{2}^{2}+\frac{1}{v+\tilde{v}}\left|\partial_{x^{\prime}} \phi_{1}\right|_{2}^{2} \\
& \leq C\left\{\left(\frac{1}{v+\tilde{v}}+\frac{v+\tilde{v}}{\gamma^{4}}\right)|\xi|^{2}|\sigma|^{2}+\frac{v^{2}}{\gamma^{4}(v+\tilde{v})}\left|\phi_{1}\right|_{2}^{2}+\left(\frac{1}{v+\tilde{v}}+\frac{v^{2}+\tilde{v}^{2}}{\gamma^{4}(v+\tilde{v})}\right)|\xi|^{2}\left|\phi_{1}\right|_{2}^{2}\right. \\
& \left.\quad+\left(\frac{\tilde{v}}{v}+1\right)\left(1+|\xi|^{2}\right) \tilde{D}_{\xi}\left[w_{1}\right]+\frac{1}{v+\tilde{v}}\left|\sqrt{\rho_{s}} \partial_{t} w_{1}\right|_{2}^{2}+\frac{v^{2}+\tilde{v}^{2}}{\gamma^{4}(v+\tilde{v})}\left|\dot{\phi}_{1}\right|_{H^{1}}^{2}\right\}
\end{aligned}
$$

Proof. We first derive the estimate for $\partial_{x^{\prime}}^{2} w_{1}^{\prime}$ and $\partial_{x^{\prime}} \phi_{1}$. We will employ the following estimate for solutions of Stokes equation. If $\left(p, h^{\prime}\right)$ is the solution of

$$
\left\{\begin{array}{l}
\nabla^{\prime} \cdot h^{\prime}=F^{0}, \\
-\Delta^{\prime} h^{\prime}+\frac{1}{v} \nabla^{\prime} p=\frac{1}{v} G^{\prime}, \\
\left.h^{\prime}\right|_{\partial D}=0,
\end{array}\right.
$$

then it holds that

$$
\left|\partial_{x^{\prime}}^{2} h^{\prime}\right|_{2}^{2}+\frac{1}{v^{2}}\left|\partial_{x^{\prime}} p\right|_{2}^{2} \leq C\left\{\left|F^{0}\right|_{H^{1}}^{2}+\frac{1}{v^{2}}\left|G^{\prime}\right|_{2}^{2}\right\} .
$$


(See, for example, [2, IV.6] and [19, III.1.5].) By the first and second equations of (4.11), with the boundary condition of $w_{1}^{\prime}$, we see that $\left(\phi_{1}, w_{1}^{\prime}\right)$ satisfies the following Stokes equation:

$$
\left\{\begin{array}{l}
\nabla^{\prime} \cdot w_{1}^{\prime}=F_{1}^{0}, \\
-\Delta^{\prime} w_{1}^{\prime}+\frac{1}{v} \nabla^{\prime}\left(\frac{P^{\prime}\left(\rho_{s}\right)}{\gamma^{2}} \phi_{1}\right)=\frac{1}{v} G_{1}^{\prime}, \\
\left.w_{1}^{\prime}\right|_{\partial D}=0,
\end{array}\right.
$$

where

$$
\begin{aligned}
F_{1}^{0}= & -\frac{1}{\gamma^{2} \rho_{s}}\left\{\partial_{t} \phi_{1}+i \xi v_{s}^{3} \phi_{1}+\gamma^{2}\left(\nabla^{\prime} \rho_{s}\right) \cdot w_{1}^{\prime}+\gamma^{2} i \xi w_{1}^{3}\right. \\
& \left.+i \xi v_{s}^{3} \sigma \phi^{(0)}+\gamma^{2} i \xi \rho_{s} \sigma w^{(0), 3}-\left\langle Q_{0} \tilde{B}_{\xi}\left(\sigma u^{(0)}+u_{1}\right)\right\rangle \phi^{(0)}\right\} \\
G_{1}^{\prime}= & -\rho_{s}\left\{\partial_{t} w_{1}^{\prime}+\frac{v}{\rho_{s}} \xi^{2} w_{1}^{\prime}-\frac{\tilde{v}}{\rho_{s}}\left(\nabla^{\prime} \cdot w_{1}^{\prime}+i \xi w_{1}^{3}\right)+i \xi v_{s}^{3} w_{1}^{\prime}\right. \\
& \left.+\nabla^{\prime}\left(\frac{1}{\rho_{s}}\right) \frac{P^{\prime}\left(\rho_{s}\right)}{\gamma^{2}} \phi_{1}-\frac{\tilde{v}}{\rho_{s}} i \xi \nabla^{\prime}\left(\sigma w^{(0), 3}\right)\right\} .
\end{aligned}
$$

By Lemma 4.6 and the Poincaré inequality, we have

$$
\begin{aligned}
\left|F_{1}^{0}\right|_{2}^{2} \leq & C\left\{\frac{1}{\gamma^{4}}|\xi|^{2}|\sigma|_{2}^{2}+\frac{1}{\gamma^{4}}\left|\phi_{1}\right|_{2}^{2}+\frac{1}{v} \tilde{D}_{\xi}\left[w_{1}\right]+\frac{1}{\gamma^{4}}\left|\dot{\phi}_{1}\right|_{2}^{2}\right\} \\
\left|\partial_{x^{\prime}} F_{1}^{0}\right|_{2}^{2} \leq & C\left\{\frac{1}{\gamma^{4}}|\xi|^{2}|\sigma|^{2}+\frac{1}{\gamma^{4}}|\xi|^{2}\left|\phi_{1}\right|_{2}^{2}+\frac{1}{v}\left(1+|\xi|^{2}\right) \tilde{D}_{\xi}\left[w_{1}\right]+\frac{1}{\gamma^{4}}\left|\dot{\phi}_{1}\right|_{H^{1}}^{2}\right\} \\
\left|G_{1}^{\prime}\right|_{2}^{2} \leq & C\left\{\frac{\tilde{v}^{2}}{\gamma^{4}}|\xi|^{2}|\sigma|^{2}+\left(\omega^{2}+\frac{\tilde{v}^{2}}{\gamma^{4}}\right)|\xi|^{2}\left|\phi_{1}\right|_{2}^{2}+\left(\frac{1}{v}+\frac{\tilde{v}^{2}}{v}\right) \tilde{D}_{\xi}\left[w_{1}\right]\right. \\
& \left.+\left(v+\frac{\tilde{v}^{2}}{v}\right)|\xi|^{2} \tilde{D}_{\xi}\left[w_{1}\right]+\frac{\tilde{v}^{2}}{\gamma^{4}}\left|\dot{\phi}_{1}\right|_{H^{1}}^{2}+\left|\sqrt{\rho_{s}} \partial_{t} w_{1}\right|_{2}^{2}\right\}
\end{aligned}
$$

Since

$$
\begin{gathered}
\partial_{x^{\prime}}\left(\frac{P^{\prime}\left(\rho_{s}\right)}{\gamma^{2}} \phi_{1}\right)= \\
\frac{P^{\prime}\left(\rho_{s}\right)}{\gamma^{2}} \partial_{x^{\prime}} \phi_{1}+\frac{P^{\prime \prime}\left(\rho_{s}\right) \partial_{x^{\prime}} \rho_{s}}{\gamma^{2}} \phi_{1}, \\
\frac{P^{\prime}\left(\rho_{s}\right)}{\gamma^{2}} \geq \frac{1}{2},
\end{gathered}
$$

and

$$
\left|\phi_{1}\right|_{2} \leq C\left|\partial_{x^{\prime}} \phi_{1}\right|_{2}
$$

by the Poincaré inequality, we see that

$$
\begin{aligned}
\left|\partial_{x^{\prime}}\left(\frac{P^{\prime}\left(\rho_{s}\right)}{\gamma^{2}} \phi_{1}\right)\right|_{2}^{2} & \geq C\left\{\left|\partial_{x^{\prime}} \phi_{1}\right|_{2}^{2}-\omega^{2}\left|\phi_{1}\right|_{2}^{2}\right\} \\
& \geq C\left(1-\omega^{2}\right)\left|\partial_{x^{\prime}} \phi_{1}\right|_{2}^{2} \\
& \geq C\left|\partial_{x^{\prime}} \phi_{1}\right|_{2}^{2}
\end{aligned}
$$


for $\omega^{2}<\frac{1}{2}$. We thus find the estimate

$$
\begin{aligned}
\left|\partial_{x^{\prime}}^{2} w_{1}^{\prime}\right|_{2}^{2}+\frac{1}{v^{2}}\left|\partial_{x^{\prime}} \phi_{1}\right|_{2}^{2} & \\
\leq & C \frac{1}{v^{2}}\left\{\frac{v^{2}+\tilde{v}^{2}}{\gamma^{4}}|\xi|^{2}|\sigma|^{2}+\frac{v^{2}}{\gamma^{4}}\left|\phi_{1}\right|_{2}^{2}+\left(\omega^{2}+\frac{v^{2}}{\gamma^{4}}+\frac{\tilde{v}^{2}}{\gamma^{4}}\right)|\xi|^{2}\left|\phi_{1}\right|_{2}^{2}\right. \\
& \left.+\left(v+\frac{1}{v}+\frac{\tilde{v}^{2}}{v}\right) \tilde{D}_{\xi}\left[w_{1}\right]+\left(v+\frac{\tilde{v}^{2}}{v}\right)|\xi|^{2} \tilde{D}_{\xi}\left[w_{1}\right]+\left|\sqrt{\rho_{s}} \partial_{t} w_{1}\right|_{2}^{2}+\frac{v^{2}+\tilde{v}^{2}}{\gamma^{4}}\left|\dot{\phi}_{1}\right|_{H^{1}}^{2}\right\} .
\end{aligned}
$$

We next derive the estimate for $\partial_{x^{\prime}}^{2} w_{1}^{3}$. The third equation of (4.11), with the boundary condition of $w_{1}^{3}$, is written as

$$
\left\{\begin{array}{l}
-\Delta^{\prime} w_{1}^{3}=G_{1}^{3}, \\
\left.w_{1}^{3}\right|_{\partial D}=0,
\end{array}\right.
$$

where

$$
\begin{aligned}
G_{1}^{3}= & -\frac{\rho_{s}}{v}\left\{\partial_{t} w_{1}^{3}+\frac{v}{\rho_{s}} \xi^{2} w_{1}^{3}-\frac{\tilde{v}}{\rho_{s}} i \xi\left(\nabla^{\prime} \cdot w_{1}^{\prime}+i \xi w_{1}^{3}\right)\right. \\
& +i \xi\left(\frac{P^{\prime}\left(\rho_{s}\right)}{\gamma^{2} \rho_{s}} \phi_{1}\right)+i \xi v_{s}^{3} w_{1}^{3}+\frac{v}{\gamma^{2} \rho_{s}^{2}} \Delta^{\prime} v_{s}^{3} \phi_{1}+w_{1}^{\prime} \cdot \nabla^{\prime} v_{s}^{3} \\
& \left.+\frac{v+\tilde{v}}{\rho_{s}} \xi^{2} \sigma w^{(0), 3}+i \xi \alpha_{0} \sigma+i \xi v_{s}^{3} \sigma w^{(0), 3}-\left\langle Q_{0} \tilde{B}_{\xi}\left(\sigma u^{(0)}+u_{1}\right)\right\rangle w^{(0), 3}\right\} .
\end{aligned}
$$

We thus obtain

$$
\left|w_{1}^{3}\right|_{H^{2}}^{2} \leq C\left|G_{1}^{3}\right|_{2}^{2}
$$

It then follows that

$$
\begin{aligned}
\left|\partial_{x^{\prime}}^{2} w_{1}^{3}\right|_{2}^{2} \leq & C \frac{1}{v^{2}}\left\{\left(1+\frac{1}{\gamma^{4}}+\frac{(v+\tilde{v})^{2}}{\gamma^{4}}\right)|\xi|^{2}|\sigma|^{2}+\frac{v^{2}}{\gamma^{4}}\left|\phi_{1}\right|_{2}^{2}+\left(1+\frac{1}{\gamma^{4}}\right)|\xi|^{2}\left|\phi_{1}\right|_{2}^{2}\right. \\
& \left.+\left(v+\tilde{v}+\frac{1}{v}\right) \tilde{D}_{\xi}\left[w_{1}\right]+\frac{\tilde{v}^{2}}{v+\tilde{v}}|\xi|^{2} \tilde{D}_{\xi}\left[w_{1}\right]+\left|\sqrt{\rho_{s}} \partial_{t} w_{1}\right|_{2}^{2}\right\}
\end{aligned}
$$

Multiplying $v^{2} /(v+\tilde{v})$ to $(4.70)+(4.71)$, we have the desired estimate. This completes the proof.

We are now in a position to prove Theorem 3.2.

PROPOSITION 4.20. Let $R>0$. There exist positive constants $v_{0}, \gamma_{0}, \omega_{0}$ and $d$ such that if $v \geq v_{0} R^{2}, \gamma^{2} /(v+\tilde{v}) \geq \gamma_{0}^{2} R^{2}$ and $(v+\tilde{v}) \omega / v \leq \omega_{0}$, then for any $l=0,1, \ldots$, there exists a constant $C=C(l)>0$ such that the estimate

$$
\begin{aligned}
& \left\|\partial_{x^{\prime}} \partial_{x_{3}}^{l} \mathcal{F}^{-1}\left[\chi_{(R)} e^{-t \widehat{L}_{\xi}} \widehat{u}_{0}\right]\right\|_{L^{2}} \\
& \quad \leq C\left\{(1+t)^{-1 / 4-l / 2}\left\|u_{0}\right\|_{L^{1}\left(\mathbb{R}: L^{2}(D)\right)}+e^{-d t}\left(\left\|u_{0}\right\|_{L^{2}}+\left\|\partial_{x^{\prime}} u_{0}\right\|_{L^{2}}\right)\right\}
\end{aligned}
$$

holds for $t \geq 0$. 
Proof. Let $b_{5}$ and $b_{6}$ be constants satisfying $b_{5}, b_{6}>1$. Define $E_{4}^{(0)}[u]$ by

$$
E_{4}^{(0)}[u]=b_{5} \frac{v}{v+\tilde{v}} \tilde{E}_{2}^{(0)}[u]+b_{6} E_{3}^{(0)}\left[u_{1}\right] .
$$

If $\gamma^{2} \geq 1$, then there exists a constant $C>0$ such that

$$
\begin{aligned}
& \frac{1}{2}\left\{\frac{1}{\gamma^{2}}|\sigma|^{2}+E_{0}\left[u_{1}\right]+\frac{1}{\gamma^{2}}\left|\partial_{x^{\prime}} \phi_{1}\right|_{2}^{2}+\tilde{D}_{\xi}\left[w_{1}\right]\right\} \\
& \quad \leq C E_{4}^{(0)} \leq \frac{3}{2}\left\{\frac{1}{\gamma^{2}}|\sigma|^{2}+E_{0}\left[u_{1}\right]+\frac{1}{\gamma^{2}}\left|\partial_{x^{\prime}} \phi_{1}\right|_{2}^{2}+\tilde{D}_{\xi}\left[w_{1}\right]\right\} .
\end{aligned}
$$

We compute $b_{5}(v /(v+\tilde{v})) \times(4.38)+b_{6} \times(4.67)+b b_{6} \times(4.13)+(4.68)$. It holds that

$$
\begin{aligned}
\frac{1}{2} \frac{d}{d t} & E_{4}^{(0)}[u]+\frac{v^{2}}{v+\tilde{v}}\left|\partial_{x^{\prime}}^{2} w_{1}\right|_{2}^{2}+\frac{1}{v+\tilde{v}}\left|\partial_{x^{\prime}} \phi_{1}\right|_{2}^{2} \\
& +\frac{b_{3} b_{5}}{4} \frac{\gamma^{2}}{v(v+\tilde{v})} \tilde{D}_{\xi}\left[w_{1}\right]+\frac{b_{5}}{2} \frac{1}{v+\tilde{v}}\left|\sqrt{\rho_{s}} \partial_{t} w_{1}\right|_{2}^{2}+b b_{6} \frac{v+\tilde{v}}{\gamma^{4}}\left|\dot{\phi}_{1}\right|_{H^{1}}^{2} \\
& +\frac{b_{6}}{2}\left\{v\left(\left|\chi_{0} \nabla^{\prime} \partial_{x^{\prime}} w_{1}\right|_{2}^{2}+|\xi|^{2}\left|\chi_{0} \partial_{x^{\prime}} w_{1}\right|_{2}^{2}\right)+\tilde{v}\left|\chi_{0}\left(\nabla^{\prime} \cdot \partial_{x^{\prime}} w_{1}^{\prime}+i \xi \partial_{x^{\prime}} w_{1}^{3}\right)\right|_{2}^{2}\right\} \\
& +\frac{b_{6}}{2} \sum_{m=1}^{N}\left\{v\left(\left|\chi_{m} \nabla^{\prime} \partial w_{1}\right|_{2}^{2}+|\xi|^{2}\left|\chi_{m} \partial w_{1}\right|_{2}^{2}\right)+\tilde{v}\left|\chi_{m}\left(\nabla^{\prime} \cdot \partial w_{1}^{\prime}+i \xi \partial w_{1}^{3}\right)\right|_{2}^{2}\right\} \\
\leq & C_{4}\left\{b_{5} \frac{v}{v+\tilde{v}}\left(\frac{1}{v}+\frac{v+\tilde{v}}{v \gamma^{2}}+\frac{\tilde{v}^{2}}{\gamma^{4}}\right)|\xi|^{2}|\sigma|^{2}+b_{5} \frac{v}{v+\tilde{v}} \frac{(v+\tilde{v})^{2}}{\gamma^{4}}|\xi|^{4}|\sigma|^{2}\right. \\
& +b_{5} \frac{v}{v+\tilde{v}}\left(\frac{1}{v}+\frac{1}{\gamma^{2}}+\frac{v^{2}}{\gamma^{4}}\right)\left|\phi_{1}\right|_{2}^{2}+b_{5} \frac{v}{v+\tilde{v}} \frac{1}{\gamma^{2}}|\xi|^{2}\left|\phi_{1}\right|_{2}^{2} \\
& +b_{6}\left(\frac{1}{\gamma^{2}}+\frac{v+\tilde{v}}{\gamma^{4}}\right)|\xi|^{2}|\sigma|^{2}+b_{6}\left(\eta+\frac{\omega^{2}}{v+\tilde{v}}+\frac{1}{\gamma^{2}}+\frac{v^{2}}{\gamma^{4}(v+\tilde{v})}\right)\left|\phi_{1}\right|_{2}^{2} \\
& +b_{6}\left(\eta+\frac{\omega^{2}}{v+\tilde{v}}+\frac{1}{\gamma^{2}}+\frac{v+\tilde{v}}{\gamma^{4}}\right)|\xi|^{2}\left|\phi_{1}\right|_{2}^{2}+b_{6}\left(\eta+\frac{1}{\gamma^{2}}\right)\left|\partial_{x^{\prime}} \phi_{1}\right|_{2}^{2} \\
& +\left(\frac{\tilde{v}}{\gamma^{4}(v+\tilde{v})}\left|\phi_{1}\right|_{2}^{2}+\left(\frac{1}{v+\tilde{v}}+\frac{v^{2}+\tilde{v}^{2}}{\gamma^{4}(v+\tilde{v})}\right)|\xi|^{2}\left|\phi_{1}\right|_{2}^{2}\right. \\
& +b_{6}\left(\frac{1}{v \eta}+\frac{v}{\gamma^{2}}+\frac{\tilde{v}}{v}+1\right) \tilde{D_{\xi}}\left[w_{1}\right]+b_{6}\left(\frac{\tilde{v}}{v}+1\right)|\xi|^{2} \tilde{D_{\xi}}\left[w_{1}\right] \\
& +b_{6} \frac{1}{v+\tilde{v}}\left|\sqrt{\rho_{s}} \partial_{t} w_{1}\right|_{2}^{2}+b b_{6} \frac{v+\tilde{v}}{\gamma^{4}}|\xi|^{2}|\sigma|^{2}+b b_{6} \frac{v+\tilde{v}}{\gamma^{4}}|\xi|^{2}\left|\phi_{1}\right|_{2}^{2} \\
& \left.+1+\frac{v+\tilde{v}}{\gamma^{4}}\right)|\xi|^{2}|\sigma|^{2} \\
& \left.+\tilde{D}_{\xi}\left[w_{1}\right]+\left(w_{1}\right]+\frac{1}{v+\tilde{v}}\left|\sqrt{\rho_{s}} \partial_{t} w_{1}\right|_{2}^{2}+\frac{v^{2}+\tilde{v}^{2}}{\gamma^{4}(v+\tilde{v})}\left|\dot{\phi}_{1}\right|_{H^{1}}^{2}\right\}
\end{aligned}
$$

Fix $b_{5}>1$ and $b_{6}>1$ sufficiently large such that $b_{6} \geq 2 C_{4} / b$ and $b_{5} \geq 8 b_{6} C_{4}$, respectively. Let us take $\eta>0$ so small satisfying $\eta \leq \min \left\{1,1 / 8 b_{6} C_{4}\right\}$. We assume that $v \geq v_{0}$ and 
$\gamma \geq \gamma_{0}$ are so large that $v \geq v_{0}>1$ and $\gamma^{2} \geq 8 b_{6} C_{4}(v+\tilde{v})$. Since we have that

$$
\begin{aligned}
\tilde{D}_{\xi}\left[w_{1}\right] & \leq C(1+R)\left|w_{1}\right|_{2}\left|\partial_{x^{\prime}}^{2} w_{1}\right|_{2} \\
& \leq \epsilon\left|\partial_{x^{\prime}}^{2} w_{1}\right|_{2}^{2}+C \frac{1}{\epsilon}(1+R)^{2}\left|w_{1}\right|_{2}^{2}
\end{aligned}
$$

for any $\epsilon>0$, if we take $\epsilon$ sufficiently small such that $\epsilon<\frac{1}{2}\left(v^{2} /(v+\tilde{v})\right)$, then we obtain

$$
\frac{d}{d t} E_{4}^{(0)}[u]+d\left(\left|\nabla^{\prime} \phi_{1}\right|_{2}^{2}+\left|\nabla^{\prime} w_{1}\right|_{H^{1}}^{2}\right) \leq C|u|_{2}^{2}
$$

Now we decompose $E_{4}^{(0)}[u]$ as

$$
E_{4}^{(0)}[u]=E_{4,0}^{(0)}[u]+E_{4,1}^{(0)}[u],
$$

where

$$
\begin{aligned}
\frac{1}{2}|u|_{2}^{2} & \leq C E_{4,0}^{(0)}[u] \leq \frac{3}{2}|u|_{2}^{2}, \\
\frac{1}{2}\left(\left|\nabla^{\prime} \phi_{1}\right|_{2}^{2}+\left|\nabla^{\prime} w_{1}\right|_{H^{1}}^{2}\right) & \leq C E_{4,1}^{(0)}[u] \leq \frac{3}{2}\left(\left|\nabla^{\prime} \phi_{1}\right|_{2}^{2}+\left|\nabla^{\prime} w_{1}\right|_{H^{1}}^{2}\right) .
\end{aligned}
$$

It then follows that

$$
\frac{d}{d t} E_{4,1}^{(0)}[u](t)+d_{1} E_{4,1}^{(0)}[u]+\frac{d}{2}\left(\left|\nabla^{\prime} \phi_{1}\right|_{2}^{2}+\left|\nabla^{\prime} w_{1}\right|_{H^{1}}^{2}\right) \leq C|u|_{2}^{2}-\frac{d}{d t} E_{4,0}^{(0)}[u](t) .
$$

We thus obtain

$$
\begin{aligned}
& E_{4,1}^{(0)}[u](t)+\frac{d}{2} \int_{0}^{t} e^{-d_{1}(t-\tau)}\left(\left|\nabla^{\prime} \phi_{1}\right|_{2}^{2}+\left|\nabla^{\prime} w_{1}\right|_{H^{1}}^{2}\right) d \tau \\
& \quad \leq e^{-d_{1} t} E_{4,1}^{(0)}\left[u_{0}\right]+C \int_{0}^{t} e^{-d_{1}(t-\tau)}|u|_{2}^{2} d \tau-\int_{0}^{t} e^{-d_{1}(t-\tau)} \frac{d}{d \tau} E_{4,0}^{(0)}[u](\tau) d \tau .
\end{aligned}
$$

Since

$$
e^{-d_{1}(t-\tau)} \frac{d}{d \tau} E_{4,0}^{(0)}[u](\tau)=\frac{d}{d \tau}\left\{e^{-d_{1}(t-\tau)} E_{4,0}^{(0)}[u](\tau)\right\}+d_{1} e^{-d_{1}(t-\tau)} E_{4,0}^{(0)}[u](\tau)
$$

and

$$
E_{4,0}^{(0)}[u] \leq C|u|_{2}^{2},
$$

we see that

$$
E_{4,1}^{(0)}[u](t) \leq e^{-d_{1} t} E_{4}^{(0)}\left[u_{0}\right]+C \int_{0}^{t} e^{-d_{1}(t-\tau)}|u(\tau)|_{2}^{2} d \tau .
$$

From (4.37), we obtain

$$
E_{4,1}^{(0)}[u](t) \leq e^{-d_{1} t} E_{4}^{(0)}\left[u_{0}\right]+C\left|u_{0}\right|_{2}^{2} \int_{0}^{t} e^{-d_{1}(t-\tau)} e^{-d_{0}|\xi|^{2} \tau} d \tau .
$$


Let us estimate the second term on the right-hand side of this inequality. We have

$$
\begin{aligned}
\int_{0}^{t / 2} \exp \left\{-d_{1}(t-\tau)-d_{0}|\xi|^{2} \tau\right\} d \tau & \leq \int_{0}^{t / 2} \exp \left\{-d_{1}(t-\tau)\right\} d \tau \\
& \leq \frac{1}{d_{1}} \exp \left\{-\frac{d_{1}}{2} t\right\} \\
& \leq \frac{1}{d_{1}} \exp \left\{-\frac{d_{1}}{2} \frac{|\xi|^{2}}{R^{2}} t\right\}, \\
\int_{t / 2}^{t} \exp \left\{-d_{1}(t-\tau)-d_{0}|\xi|^{2} \tau\right\} d \tau & \leq \exp \left\{-\frac{d_{0}}{2}|\xi|^{2} t\right\} \int_{t / 2}^{t} \exp \left\{-d_{1}(t-\tau)\right\} d \tau \\
& \leq \frac{1}{d_{1}} \exp \left\{-\frac{d_{0}}{2}|\xi|^{2} t\right\} .
\end{aligned}
$$

We set $d_{2}=\min \left\{d_{0}, d_{1} / R^{2}\right\}$. It then follows that there exist positive constants $v_{0}, \gamma_{0}, \omega_{0}, d_{1}$ and $d_{2}$ such that if $v \geq v_{0} R^{2}, \gamma^{2} /(v+\tilde{v}) \geq \gamma_{0}^{2} R^{2}$ and $(v+\tilde{v}) \omega / v \leq \omega_{0}$, then

$$
E_{4,1}^{(0)}[u](t) \leq C\left\{e^{-d_{2} / 2|\xi|^{2} t}\left|u_{0}\right|_{2}^{2}+e^{-d_{1} t} E_{4}^{(0)}\left[u_{0}\right]\right\} .
$$

Combining Propositions 4.12 and 4.20 with $R=1$ we obtain the desired estimates in Theorem 3.2.

\section{Decay estimate of the high-frequency part}

In this section we will give a proof of Theorem 3.3. To prove Theorem 3.3, we will employ an energy method to obtain the estimate on solutions of

$$
\partial_{t} u+\widehat{L}_{\xi} u=0,\left.\quad w\right|_{\partial \Omega}=0,\left.\quad u\right|_{t=0}=u_{0}
$$

similarly to Section 4. The following Propositions 5.1-5.6 can be proved in a similar manner in Section 4. So we give the statements only and omit the proofs.

PROPOSITION 5.1. There exists a constant $v_{0}>0$ such that if $v \geq v_{0}$, then the following estimates hold:

$$
\begin{gathered}
\frac{1}{2} \frac{d}{d t} E_{0}[u]+\frac{1}{2} \tilde{D}_{\xi}[w] \leq C \frac{v}{\gamma^{4}}|\phi|_{2}^{2}, \\
\frac{v+\tilde{v}}{\gamma^{4}}|\dot{\phi}|_{2}^{2} \leq C\left(1+\frac{v+\tilde{v}}{v} \omega^{2}\right) \tilde{D}_{\xi}[w] .
\end{gathered}
$$

We proceed to estimate derivatives of $u$. We introduce some notation. We define $J_{2}^{(\infty)}[u]$ by

$$
J_{2}^{(\infty)}[u]=-2 \operatorname{Re}\left\langle u, \widehat{B}_{\xi} \tilde{Q} u\right\rangle .
$$

In addition, we set

$$
\begin{gathered}
E_{2}^{(\infty)}[u]=\left(1+\frac{\tilde{b}_{3} \gamma^{2}}{v}\right) E_{0}[u]+\tilde{D}_{\xi}[w], \\
\tilde{E}_{2}^{(\infty)}[u]=E_{2}^{(\infty)}[u]+J_{2}^{(\infty)}[u],
\end{gathered}
$$


where $\tilde{b}_{3}$ is a positive constant to be determined later. We note that there exists a constant $\tilde{b}_{3}^{*}>0$ such that if $\tilde{b}_{3} \geq \tilde{b}_{3}^{*}$ and $\gamma^{2} \geq 1$, then

$$
\frac{1}{2} E_{2}^{(\infty)}[u] \leq \tilde{E}_{2}^{(\infty)}[u] \leq \frac{3}{2} E_{2}^{(\infty)}[u]
$$

Taking $\tilde{b}_{3}$ suitably large, we have the following estimate for $\tilde{E}_{2}^{(\infty)}[u]$.

Proposition 5.2. There exist constants $\tilde{b}_{3} \geq \tilde{b}_{3}^{*}$ and $v_{0}>0$ such that if $v \geq v_{0}$ and $\gamma^{2} \geq 1$, then the following estimate holds:

$$
\frac{1}{2} \frac{d}{d t} \tilde{E}_{2}^{(\infty)}[u]+\frac{1}{4} \tilde{b}_{3} \frac{\gamma^{2}}{v} \tilde{D}_{\xi}[w]+\frac{1}{2}\left|\sqrt{\rho_{s}} \partial_{t} w\right|_{2}^{2} \leq C\left\{\left(\frac{1}{\gamma^{2}}+\frac{v^{2}}{\gamma^{4}}\right)|\phi|_{2}^{2}+\frac{1}{\gamma^{2}}|\xi|^{2}|\phi|_{2}^{2}\right\} .
$$

PROPOSITION 5.3. For $1 \leq m \leq N$, there exist constants $v_{0}>0$ and $b>0$ such that if $v \geq v_{0}, \gamma^{2} \geq 1$ and $\omega \leq 1$, then the following estimate holds:

$$
\begin{aligned}
\frac{1}{2} \frac{d}{d t} & \left(\frac{1}{\gamma^{2}}\left|\chi_{m} \sqrt{\frac{P^{\prime}\left(\rho_{s}\right)}{\gamma^{2} \rho_{s}}} \partial \phi\right|_{2}^{2}+\left|\chi_{m} \sqrt{\rho_{s}} \partial w\right|_{2}^{2}\right)+b \frac{v+\tilde{v}}{\gamma^{4}}\left|\chi_{m} \partial \dot{\phi}\right|_{2}^{2} \\
& +\frac{1}{2} \nu\left(\left|\chi_{m} \nabla^{\prime} \partial w\right|_{2}^{2}+|\xi|^{2}\left|\chi_{m} \partial w\right|_{2}^{2}\right)+\frac{1}{2} \tilde{v}\left|\chi_{m}\left(\nabla^{\prime} \cdot \partial w^{\prime}+i \xi \partial w^{3}\right)\right|_{2}^{2} \\
\leq & C\left\{\left(\eta+\frac{1}{\gamma^{2}}\right)|\phi|_{2}^{2}+\left(\eta+\frac{1}{\gamma^{2}}+\frac{v+\tilde{v}}{\gamma^{4}}\right)|\xi|^{2}|\phi|_{2}^{2}+\left(\eta+\frac{1}{\gamma^{2}}\right)\left|\partial_{x^{\prime}} \phi\right|_{2}^{2}\right. \\
& \left.+\left(\frac{1}{\eta v}+\frac{v}{\gamma^{2}}+\frac{\tilde{v}}{v}+1\right) \tilde{D}_{\xi}[w]\right\}
\end{aligned}
$$

for any $\eta>0$ with $C$ independent of $\eta$.

Proposition 5.4. For $1 \leq m \leq N$, there exist constants $v_{0}>0$ and $b>0$ such that if $v \geq v_{0}, \gamma^{2} \geq 1$ and $\omega \leq 1$, then the following estimate holds:

$$
\begin{aligned}
\frac{1}{2} & \frac{d}{d t}\left(\frac{1}{\gamma^{2}}\left|\chi_{m} \sqrt{\frac{P^{\prime}\left(\rho_{s}\right)}{\gamma^{2} \rho_{s}}} \partial_{n} \phi\right|_{2}^{2}\right)+\frac{1}{2} \frac{1}{v+\tilde{v}}\left|\chi_{m} \frac{P^{\prime}\left(\rho_{s}\right)}{\gamma^{2}} \partial_{n} \phi\right|_{2}^{2}+b \frac{v+\tilde{v}}{\gamma^{4}}\left|\chi_{m} \partial_{n} \dot{\phi}\right|_{2}^{2} \\
\leq & C\left\{\left(\frac{\omega^{2}}{v+\tilde{v}}+\frac{v^{2}}{\gamma^{4}(v+\tilde{v})}\right)|\phi|_{2}^{2}+\frac{v+\tilde{v}}{\gamma^{4}}|\xi|^{2}|\phi|_{2}^{2}+\left(\frac{\tilde{v}}{v}+1\right) \tilde{D}_{\xi}[w]\right. \\
& \left.+\frac{v}{v+\tilde{v}}|\xi|^{2} \tilde{D}_{\xi}[w]+\frac{v^{2}}{v+\tilde{v}}\left(\left|\chi_{m} \partial_{n} \partial w\right|_{2}^{2}+\left|\chi_{m} \partial^{2} w\right|_{2}^{2}\right)+\frac{1}{v+\tilde{v}}\left|\sqrt{\rho_{s}} \partial_{t} w\right|_{2}^{2}\right\}
\end{aligned}
$$


Proposition 5.5. There exist constants $v_{0}>0$ and $b>0$ such that if $v \geq v_{0}, \gamma^{2} \geq 1$ and $\omega \leq 1$, then the following estimate holds:

$$
\begin{aligned}
\frac{1}{2} \frac{d}{d t} & \left(\frac{1}{\gamma^{2}}\left|\chi_{0} \sqrt{\frac{P^{\prime}\left(\rho_{s}\right)}{\gamma^{2} \rho_{s}}} \partial_{x^{\prime}} \phi\right|_{2}^{2}+\left|\chi_{0} \sqrt{\rho_{s}} \partial_{x^{\prime}} w\right|_{2}^{2}\right)+b \frac{v+\tilde{v}}{\gamma^{4}}\left|\chi_{0} \partial_{x^{\prime}} \dot{\phi}\right|_{2}^{2} \\
& +\frac{1}{2} v\left(\left|\chi_{0} \nabla^{\prime} \partial_{x^{\prime}} w\right|_{2}^{2}+|\xi|^{2}\left|\chi_{0} \partial_{x^{\prime}} w\right|_{2}^{2}\right)+\frac{1}{2} \tilde{v}\left|\chi_{0}\left(\nabla^{\prime} \cdot \partial_{x^{\prime}} w^{\prime}+i \xi \partial_{x^{\prime}} w^{3}\right)\right|_{2}^{2} \\
\leq & C\left\{\frac{1}{\gamma^{2}}|\phi|_{2}^{2}+\left(\frac{1}{\gamma^{2}}+\frac{v+\tilde{v}}{\gamma^{4}}+\frac{\omega^{2}}{v+\tilde{v}}\right)|\xi|^{2}|\phi|_{2}^{2}+\left(\eta+\frac{1}{\gamma^{2}}\right)\left|\partial_{x^{\prime}} \phi\right|_{2}^{2}\right. \\
& \left.+\left(\frac{1}{\eta v}+\frac{v}{\gamma^{2}}+\frac{\tilde{v}}{v}+1\right) \tilde{D}_{\xi}[w]\right\}
\end{aligned}
$$

for any $\eta>0$ with $C$ independent of $\eta$.

Before proceeding further we introduce an energy functional. We define $E_{3}^{(\infty)}[u]$ by

$$
\begin{aligned}
E_{3}^{(\infty)}[u]= & \frac{1}{\gamma^{2}}\left|\chi_{0} \sqrt{\frac{P^{\prime}\left(\rho_{s}\right)}{\gamma^{2} \rho_{s}}} \partial_{x^{\prime}} \phi\right|_{2}^{2}+\left|\chi_{0} \sqrt{\rho_{s}} \partial_{x^{\prime}} w\right|_{2}^{2} \\
& +\tilde{b}_{4} \sum_{m=1}^{N}\left(\frac{1}{\gamma^{2}}\left|\chi_{m} \sqrt{\frac{P^{\prime}\left(\rho_{s}\right)}{\gamma^{2} \rho_{s}}} \partial \phi\right|_{2}^{2}+\left|\chi_{m} \sqrt{\rho_{s}} \partial w\right|_{2}^{2}\right)+\sum_{m=1}^{N} \frac{1}{\gamma^{2}}\left|\chi_{m} \sqrt{\frac{P^{\prime}\left(\rho_{s}\right)}{\gamma^{2} \rho_{s}}} \partial_{n} \phi\right|_{2}^{2},
\end{aligned}
$$

where $\tilde{b}_{4}$ is a positive constant. Taking $\tilde{b}_{4}$ suitably large, we have the following estimate for $E_{3}^{(\infty)}[u]$.

PROPOSITION 5.6. There exist constants $v_{0}>0, b>0$ and $\tilde{b}_{4}>0$ such that if $v \geq v_{0}$, $\gamma^{2} \geq 1$ and $\omega \leq 1$, then the following estimate holds:

$$
\begin{aligned}
& \frac{1}{2} \frac{d}{d t} E_{3}^{(\infty)}[u]+b \frac{v+\tilde{v}}{\gamma^{4}}\left|\partial_{x^{\prime}} \dot{\phi}\right|_{2}^{2} \\
& \quad+\frac{1}{2}\left\{v\left(\left|\chi_{0} \nabla^{\prime} \partial_{x^{\prime}} w\right|_{2}^{2}+|\xi|^{2}\left|\chi_{0} \partial_{x^{\prime}} w\right|_{2}^{2}\right)+\tilde{v}\left|\chi_{0}\left(\nabla^{\prime} \cdot \partial_{x^{\prime}} w^{\prime}+i \xi \partial_{x^{\prime}} w^{3}\right)\right|_{2}^{2}\right\} \\
& \quad+\frac{1}{2} \sum_{m=1}^{N}\left\{v\left(\left|\chi_{m} \nabla^{\prime} \partial w\right|_{2}^{2}+|\xi|^{2}\left|\chi_{m} \partial w\right|_{2}^{2}\right)+\tilde{v}\left|\chi_{m}\left(\nabla^{\prime} \cdot \partial w^{\prime}+i \xi \partial w^{3}\right)\right|_{2}^{2}\right\} \\
& \leq C\left\{\left(\eta+\frac{\omega^{2}}{v+\tilde{v}}+\frac{1}{\gamma^{2}}+\frac{v^{2}}{\gamma^{4}(v+\tilde{v})}\right)|\phi|_{2}^{2}+\left(\eta+\frac{\omega^{2}}{v+\tilde{v}}+\frac{1}{\gamma^{2}}+\frac{v+\tilde{v}}{\gamma^{4}}\right)|\xi|^{2}|\phi|_{2}^{2}\right. \\
&+\left(\eta+\frac{1}{\gamma^{2}}\right)\left|\partial_{x^{\prime}} \phi\right|_{2}^{2}+\left(\frac{1}{v \eta}+\frac{v}{\gamma^{2}}+\frac{\tilde{v}}{v}+1\right) \tilde{D}_{\xi}[w] \\
&\left.+\frac{v}{v+\tilde{v}}|\xi|^{2} \tilde{D}_{\xi}[w]+\frac{1}{v+\tilde{v}}\left|\sqrt{\rho_{s}} \partial_{t} w\right|_{2}^{2}\right\}
\end{aligned}
$$

for any $\eta>0$ with $C$ independent of $\eta$.

We do not have the estimate for $\phi$ such as $|\phi|_{2} \leq C\left|\partial_{x^{\prime}} \phi\right|_{2}$ similar to that for $\phi_{1}$ in Section 4 . We thus use the estimate for a solution of the Fourier transformed Stokes equation of the case $|\xi|^{2} \gg 1$. 
Proposition 5.7. Assume that $(p, h) \in H^{1}(D) \times H^{2}(D)$ is a solution of the following Stokes equation:

$$
\left\{\begin{array}{l}
\nabla^{\prime} \cdot h^{\prime}+i \xi h^{3}=F^{0} \\
\left(|\xi|^{2}-\Delta^{\prime}\right) h^{\prime}+\frac{1}{v} \partial_{x^{\prime}} p=\frac{1}{v} G^{\prime} \\
\left(|\xi|^{2}-\Delta^{\prime}\right) h^{3}+\frac{1}{v} i \xi p=\frac{1}{v} G^{3} \\
\left.h\right|_{\partial D}=0 .
\end{array}\right.
$$

There exists a constant $R_{0}=R_{0}(D)>0$ such that if $|\xi| \geq R_{0}$, then the following estimate holds:

$$
\begin{aligned}
& \frac{1}{v^{2}}|p|_{2}^{2}+\frac{1}{v^{2}}|\xi|^{2}|p|_{2}^{2}+\frac{1}{v^{2}}\left|\partial_{x^{\prime}} p\right|_{2}^{2}+|h|_{2}^{2}+|\xi|^{2}|h|_{2}^{2}+\left|\partial_{x^{\prime}} h\right|_{2}^{2}+\sum_{j=0}^{2}|\xi|^{2 j}\left|\partial_{x^{\prime}}^{2-j} h\right|_{2}^{2} \\
& \leq C R_{0}^{2}\left\{\left|F^{0}\right|_{2}^{2}+|\xi|^{2}\left|F^{0}\right|_{2}^{2}+\left|\partial_{x^{\prime}} F^{0}\right|_{2}^{2}+\frac{1}{v^{2}}|G|_{2}^{2}+\left|\partial_{x^{\prime}} h\right|_{2}^{2}\right\},
\end{aligned}
$$

where $C$ is a positive constant independent of $|\xi|$.

Proposition 5.7 can be proved similarly to the proof of [6, Lemma 6.6] and we omit the proof. Applying Proposition 5.7, we have the following estimate.

PROPOSITION 5.8. There exist constant $v_{0}>0$ such that if $v \geq v_{0}, \gamma^{2} \geq 1$ and $\omega \leq 1$, then the following estimate holds:

$$
\begin{aligned}
& \frac{1}{v+\tilde{v}}\left(|\phi|_{2}^{2}+|\xi|^{2}|\phi|_{2}^{2}+\left|\partial_{x^{\prime}} \phi\right|_{2}^{2}\right) \\
& \quad+\frac{v^{2}}{v+\tilde{v}}\left(|w|_{2}^{2}+|\xi|^{2}|w|_{2}^{2}+\left|\partial_{x^{\prime}} w\right|_{2}^{2}+\sum_{j=0}^{2}|\xi|^{2 j}\left|\partial_{x^{\prime}}^{2-j} w\right|_{2}^{2}\right) \\
& \leq C R_{0}^{2}\left\{\left(\frac{\omega^{2}}{v+\tilde{v}}+\frac{v^{2}}{\gamma^{4}(v+\tilde{v})}\right)|\phi|_{2}^{2}+\frac{v}{v+\tilde{v}} \tilde{D}_{\xi}[w]\right. \\
& \left.\quad+\frac{v^{2}+\tilde{v}^{2}}{\gamma^{4}(v+\tilde{v})}\left(|\dot{\phi}|_{2}^{2}+|\xi|^{2}|\dot{\phi}|_{2}^{2}+\left|\partial_{x^{\prime}} \dot{\phi}\right|_{2}^{2}\right)+\frac{1}{v+\tilde{v}}\left|\sqrt{\rho_{s}} \partial_{t} w\right|_{2}^{2}\right\}
\end{aligned}
$$

for $|\xi| \geq R_{0}$, where $R_{0}$ is the constant given in Proposition 5.7 and $C$ is a positive constant independent of $|\xi|$.

Proof. We observe that $(\phi, w)$ satisfies the following Stokes equation:

$$
\left\{\begin{array}{l}
\nabla^{\prime} \cdot w^{\prime}+i \xi w^{3}=F^{0} \\
\left(\xi^{2}-\Delta^{\prime}\right) w^{\prime}+\frac{1}{v} \nabla^{\prime}\left(\frac{P^{\prime}\left(\rho_{s}\right)}{\gamma^{2}} \phi\right)=\frac{1}{v} G^{\prime}, \\
\left(\xi^{2}-\Delta^{\prime}\right) w^{3}+\frac{1}{v} i \xi \frac{P^{\prime}\left(\rho_{s}\right)}{\gamma^{2}} \phi=\frac{1}{v} G^{3}, \\
\left.w\right|_{\partial D}=0
\end{array}\right.
$$


where

$$
\begin{aligned}
F^{0} & =-\frac{1}{\rho_{s}}\left\{\partial_{t} \phi+i \xi v_{s}^{3} \phi+\left(\nabla^{\prime} \rho_{s}\right) \cdot w^{\prime}\right\}, \\
G^{\prime} & =-\rho_{s}\left\{\partial_{t} w^{\prime}-\frac{\tilde{v}}{\rho_{s}} \nabla^{\prime}\left(\nabla^{\prime} \cdot w^{\prime}+i \xi w^{3}\right)-\frac{P^{\prime}\left(\rho_{s}\right)}{\gamma^{2} \rho_{s}} \phi \nabla^{\prime} \rho_{s}+i \xi v_{s}^{3} w^{\prime}\right\}, \\
G^{3} & =-\rho_{s}\left\{\partial_{t} w^{3}-\frac{\tilde{v}}{\rho_{s}} i \xi\left(\nabla^{\prime} \cdot w^{\prime}+i \xi w^{3}\right)+i \xi v_{s}^{3} w^{3}+\frac{v}{\gamma^{2} \rho_{s}} \Delta^{\prime} v_{s}^{3} \phi+w^{\prime} \cdot \nabla^{\prime} v_{s}^{3}\right\} .
\end{aligned}
$$

Therefore, we get the desired estimate from Proposition 5.7. This completes the proof.

We finally prove Theorem 3.3.

Proof of Theorem 3.3. Let $\tilde{b}_{5}, \tilde{b}_{6}$ and $\tilde{b}_{7}$ be constants satisfying $\tilde{b}_{5}, \tilde{b}_{6}, \tilde{b}_{7}>1$. Define $\tilde{E}_{4}^{(\infty)}[u]$ by

$$
\tilde{E}_{4}^{(\infty)}[u]=\tilde{b}_{5} E_{3}^{(\infty)}[u]+\frac{\tilde{b}_{6}}{v+\tilde{v}} \tilde{E}_{2}^{(\infty)}[u]+\tilde{b}_{7}\left(1+\frac{\tilde{v}}{v}\right)\left(1+|\xi|^{2}\right) E_{0}[u] .
$$

We compute

$$
\begin{aligned}
(5.8) & +\tilde{b}_{5} \times\left\{(5.7)+b \frac{v+\tilde{v}}{\gamma^{4}}\left(1+|\xi|^{2}\right)|\dot{\phi}|_{2}^{2}\right\}+\frac{\tilde{b}_{6}}{v+\tilde{v}} \times \\
& +\tilde{b}_{7}\left(1+\frac{\tilde{v}}{v}\right)\left(1+|\xi|^{2}\right) \times(5.1)
\end{aligned}
$$

then

$$
\begin{aligned}
& \frac{1}{2} \frac{d}{d t} \tilde{E}_{4}^{(\infty)}[u]+\frac{v^{2}}{v+\tilde{v}}\left(|w|_{2}^{2}+|\xi|^{2}|w|_{2}^{2}+\left|\partial_{x^{\prime}} w\right|_{2}^{2}+\sum_{j=0}^{2}|\xi|^{2 j}\left|\partial_{x^{\prime}}^{j} w\right|_{2}^{2}\right) \\
& \quad+\frac{1}{v+\tilde{v}}\left(|\phi|_{2}^{2}+|\xi|^{2}|\phi|_{2}^{2}+\left|\partial_{x^{\prime}} \phi\right|_{2}^{2}\right)+b \tilde{b}_{5} \frac{v+\tilde{v}}{\gamma^{4}}\left(|\dot{\phi}|_{2}^{2}+|\xi|^{2}|\dot{\phi}|_{2}^{2}+\left|\partial_{x^{\prime}} \dot{\phi}\right|_{2}^{2}\right) \\
& \quad+\frac{\tilde{b}_{5}}{2}\left\{v\left(\left|\chi_{0} \nabla^{\prime} \partial_{x^{\prime}} w\right|_{2}^{2}+|\xi|^{2}\left|\chi_{0} \partial_{x^{\prime}} w\right|_{2}^{2}\right)+\tilde{v}\left|\chi_{0}\left(\nabla^{\prime} \cdot \partial_{x^{\prime}} w^{\prime}+i \xi \partial_{x^{\prime}} w^{3}\right)\right|_{2}^{2}\right\} \\
& \quad+\frac{\tilde{b}_{5}}{2} \sum_{m=1}^{N}\left\{v\left(\left|\chi_{m} \nabla^{\prime} \partial w\right|_{2}^{2}+|\xi|^{2}\left|\chi_{m} \partial w\right|_{2}^{2}\right)+\tilde{v}\left|\chi_{m}\left(\nabla^{\prime} \cdot \partial w^{\prime}+i \xi \partial w^{3}\right)\right|_{2}^{2}\right\} \\
& \quad+\frac{\tilde{b}_{3} \tilde{b}_{6}}{4} \frac{\gamma^{2}}{v(v+\tilde{v})} \tilde{D}_{\xi}[w]+\frac{\tilde{b}_{6}}{2} \frac{1}{v+\tilde{v}}\left|\sqrt{\rho_{s}} \partial_{t} w\right|_{2}^{2}+\frac{\tilde{b}_{7}}{2}\left(1+\frac{\tilde{v}}{v}\right)\left(1+|\xi|^{2}\right) \tilde{D}_{\xi}[w] \\
& \leq \tilde{C}_{4}\left\{R_{0}^{2}\left(\frac{\omega^{2}}{v+\tilde{v}}+\frac{v^{2}}{\gamma^{4}(v+\tilde{v})}\right)|\phi|_{2}^{2}+R_{0}^{2} \frac{v}{v+\tilde{v}} \tilde{D}_{\xi}[w]\right. \\
&+R_{0}^{2} \frac{v^{2}+\tilde{v}^{2}}{\gamma^{4}(v+\tilde{v})}\left(|\dot{\phi}|_{2}^{2}+|\xi|^{2}|\dot{\phi}|_{2}^{2}+\left|\partial_{x^{\prime}} \dot{\phi}\right|_{2}^{2}\right) \\
&+R_{0}^{2} \frac{1}{v+\tilde{v}}\left|\sqrt{\rho_{s}} \partial_{t} w\right|_{2}^{2}+\tilde{b}_{5}\left(\eta+\frac{\omega^{2}}{v+\tilde{v}}+\frac{1}{\gamma^{2}}+\frac{v^{2}}{\gamma^{4}(v+\tilde{v})}\right)|\phi|_{2}^{2}
\end{aligned}
$$




$$
\begin{aligned}
& +\tilde{b}_{5}\left(\eta+\frac{\omega^{2}}{v+\tilde{v}}+\frac{1}{\gamma^{2}}+\frac{v+\tilde{v}}{\gamma^{4}}\right)|\xi|^{2}|\phi|_{2}^{2}+\tilde{b}_{5}\left(\eta+\frac{1}{\gamma^{2}}\right)\left|\partial_{x^{\prime}} \phi\right|_{2}^{2} \\
& +\tilde{b}_{5}\left(\frac{1}{v \eta}+\frac{v}{\gamma^{2}}+\frac{\tilde{v}}{v}+1\right) \tilde{D}_{\xi}[w]+\tilde{b}_{5} \frac{v}{v+\tilde{v}}|\xi|^{2} \tilde{D}_{\xi}[w] \\
& +\tilde{b}_{5} \frac{1}{v+\tilde{v}}\left|\sqrt{\rho_{s}} \partial_{t} w\right|_{2}^{2}+b \tilde{b}_{5}\left(\frac{v+\tilde{v}}{\gamma^{4}}+\frac{(v+\tilde{v})^{2}}{\gamma^{4} v}\right)\left(1+|\xi|^{2}\right) \tilde{D}_{\xi}[w] \\
& +\tilde{b}_{6}\left(\frac{1}{\gamma^{2}(v+\tilde{v})}+\frac{v^{2}}{\gamma^{4}(v+\tilde{v})}\right)|\phi|_{2}^{2} \\
& \left.+\tilde{b}_{6} \frac{1}{\gamma^{2}(v+\tilde{v})}|\xi|^{2}|\phi|_{2}^{2}+\tilde{b}_{7} \frac{v+\tilde{v}}{\gamma^{4}}\left(1+|\xi|^{2}\right)|\phi|_{2}^{2}\right\} .
\end{aligned}
$$

Fix $\tilde{b}_{5}>1, \tilde{b}_{6}>1$ and $\tilde{b}_{7}>1$ so large that $\tilde{b}_{5} \geq\left(2 \tilde{C}_{4} / b\right) R_{0}^{2}, \tilde{b}_{6} \geq 8 \tilde{C}_{4} \max \left\{R_{0}^{2}, \tilde{b}_{5}\right\}$ and $\tilde{b}_{7}>20 \tilde{C}_{4} \max \left\{R_{0}^{2}, \tilde{b}_{5}(1 / \eta(v+\tilde{v})), \tilde{b}_{5}, b \tilde{b}_{5}\right\}$, respectively. We take $\eta>0$ and $\omega>$ 0 sufficiently small such that $\eta<\left(1 / 20 \tilde{C}_{4} \tilde{b}_{5}\right)(1 /(v+\tilde{v}))$ and $\omega^{2}<\left(1 / 20 \tilde{C}_{4}\right) \min \left\{1 / R_{0}^{2}\right.$, $\left.1 / \tilde{b}_{5}\right\}$, respectively. We assume that $v \geq v_{0}$ and $\gamma \geq \gamma_{0}$ are large enough such that $v \geq v_{0}>1$ and $\gamma^{2}>20 \tilde{C}_{4} \max \left\{\tilde{b}_{6}(v+\tilde{v}),\left(\tilde{b}_{5} / \tilde{b}_{7}\right)\left(v^{2} /(v+\tilde{v})\right), \sqrt{b_{7}}(v+\tilde{v})\right\}$. We then arrive at the estimate

$$
\begin{aligned}
& \frac{d}{d t} \tilde{E}_{4}^{(\infty)}[u]+\frac{v^{2}}{v+\tilde{v}}\left(|w|_{2}^{2}+|\xi|^{2}|w|_{2}^{2}+\left|\partial_{x^{\prime}} w\right|_{2}^{2}+\sum_{j=1}^{2}|\xi|^{2 j}\left|\partial_{x^{\prime}}^{j} w\right|_{2}^{2}\right) \\
& \quad+\frac{1}{v+\tilde{v}}\left(|\phi|_{2}^{2}+|\xi|^{2}|\phi|_{2}^{2}+\left|\partial_{x^{\prime}} \phi\right|_{2}^{2}\right)+\frac{v+\tilde{v}}{\gamma^{4}}\left(|\dot{\phi}|_{2}^{2}+|\xi|^{2}|\dot{\phi}|_{2}^{2}+|\dot{\phi}|_{H^{1}}^{2}\right) \\
& \quad+v\left(\left|\chi_{0} \nabla^{\prime} \partial_{x^{\prime}} w\right|_{2}^{2}+|\xi|^{2}\left|\chi_{0} \partial_{x^{\prime}} w\right|_{2}^{2}\right)+\tilde{v}\left|\chi_{0}\left(\nabla^{\prime} \cdot \partial_{x^{\prime}} w^{\prime}+i \xi \partial_{x^{\prime}} w^{3}\right)\right|_{2}^{2} \\
& \quad+\sum_{m=1}^{N}\left\{v\left(\left|\chi_{m} \nabla^{\prime} \partial w\right|_{2}^{2}+|\xi|^{2}\left|\chi_{m} \partial w\right|_{2}^{2}\right)+\tilde{v}\left|\chi_{m}\left(\nabla^{\prime} \cdot \partial w^{\prime}+i \xi \partial w^{3}\right)\right|_{2}^{2}\right\} \\
& \quad+\frac{1}{v+\tilde{v}}\left|\sqrt{\rho_{s}} \partial_{t} w\right|_{2}^{2}+\frac{v+\tilde{v}}{v}\left(1+|\xi|^{2}\right) \tilde{D}_{\xi}[w] \\
& \leq 0
\end{aligned}
$$

for all $\xi \in \mathbb{R}$ with $|\xi| \geq R_{0}$. We define $E_{4}^{(\infty)}[u]$ by

$$
E_{4}^{(\infty)}[u]=|\phi|_{2}^{2}+|\xi|^{2}|\phi|_{2}^{2}+\left|\partial_{x^{\prime}} \phi\right|_{2}^{2}+|w|_{2}^{2}+|\xi|^{2}|w|_{2}^{2}+\left|\partial_{x^{\prime}} w\right|_{2}^{2}
$$

Since

$$
\begin{gathered}
\frac{1}{2}\left\{\left(1+\frac{\tilde{b}_{3} \gamma^{2}}{v}\right) E_{0}[u]+\tilde{D}_{\xi}[w]\right\} \leq \tilde{E}_{2}^{(\infty)}[u] \leq \frac{3}{2}\left\{\left(1+\frac{\tilde{b}_{3} \gamma^{2}}{v}\right) E_{0}[u]+\tilde{D}_{\xi}[w]\right\}, \\
\frac{1}{2} \frac{1}{\gamma^{2}}\left|\partial_{x^{\prime}} \phi\right|_{2}^{2} \leq \tilde{C}_{5} E_{3}^{(\infty)}[u] \leq \frac{3}{2}\left(\frac{1}{\gamma^{2}}\left|\partial_{x^{\prime}} \phi\right|_{2}^{2}+\left|\partial_{x^{\prime}} w\right|_{2}^{2}\right)
\end{gathered}
$$

for a positive constant $\tilde{C}_{5}$, we see that

$$
\frac{1}{2} E_{4}^{(\infty)}[u] \leq \tilde{C}_{6} \tilde{E}_{4}^{(\infty)}[u] \leq \frac{3}{2} E_{4}^{(\infty)}[u]
$$


for a positive constant $\tilde{C}_{6}$. We thus see that there exist positive constants $\nu_{0}, \gamma_{0}, \omega_{0}$ and $d$ such that if $v \geq v_{0}, \gamma^{2} /(v+\tilde{v}) \geq \gamma_{0}^{2} R_{0}^{2}$ and $\omega \leq \omega_{0} R_{0}^{-2}$, then

$$
E_{4}^{(\infty)}[u](t) \leq C e^{-d t} E_{4}^{(\infty)}\left[u_{0}\right]
$$

for $|\xi| \geq R_{0}$. On the other hand, for $1 \leq|\xi| \leq R_{0}$, we obtain the desired estimate from (4.72) with $R=R_{0}$. This completes the proof.

Acknowledgement. The author would like to thank Professor Yoshiyuki Kagei for his useful comments and constant encouragement.

\section{REFERENCES}

[1] K. Deckelnick. Decay estimates for the compressible Navier-Stokes equations in unbounded domain. Math. Z. 209 (1992), 115-130.

[2] G. P. Galdi. An Introduction to the Mathematical Theory of the Navier-Stokes Equations I, Linearized Steady Problems (Springer Tracts in Natural Philosophy, 38). Springer, Berlin, 1994.

[3] D. Hoff and K. Zumbrun. Multi-dimensional diffusion waves for the Navier-Stokes equations of compressible flow. Indiana Univ. Math. J. 44 (1995), 604-676.

[4] G. Iooss and M. Padula. Structure of the linearized problem for compressible parallel fluid flows. Ann. Univ. Ferrara Sez. VII Sci. Mat. 43 (1998), 157-171.

[5] Y, Ishihara and Y. Kagei. Large time behavior of the semigroup on $L^{p}$ spaces associated with the linearized compressible Navier-Stokes equation in a cylindrical domain. J. Differential Equations 248 (2010), 252286.

[6] Y. Kagei. Asymptotic behavior of solutions of the compressible Navier-Stokes equation around the plane Couette flow. J. Math. Fluid Mech. 13 (2011), 1-31.

[7] Y. Kagei. Asymptotic behavior of solutions to the compressible Navier-Stokes equation around a parallel flow. Arch. Ration. Mech. Anal. 205 (2012), 585-650.

[8] Y. Kagei and T. Kobayashi. Asymptotic behavior of solutions to the compressible Navier-Stokes equations on the half space. Arch. Ration. Mech. Anal. 177 (2005), 231-330.

[9] Y. Kagei, Y. Nagahuchi and T. Sudou. Decay estimate on solutions of the linearized compressible NavierStokes equation around a Poiseuille type flow. J. Math-for-Ind. 2A (2010), 39-56.

[10] Y. Kagei, Y. Nagahuchi and T. Sudou. Correction to 'Decay estimate on solutions of the linearized compressible Navier-Stokes equation around a Poiseuille type flow in J. Math-for-Ind. 2A (2010), pp. 39-56'. J. Math-for-Ind. 2B (2010), 235.

[11] Y. Kagei and T. Nukumizu. Asymptotic behavior of solutions to the compressible Navier-Stokes equation in a cylindrical domain. Osaka J. Math. 45 (2008), 987-1026.

[12] S. Kawashima. Systems of a hyperbolic-parabolic composite type, with applications to the equations of magnethydrodynamics. PhD Thesis, Kyoto University, 1983.

[13] T. Kobayashi. Some estimates of solutions for the equations of motion of compressible viscous fluid in an exterior domain in $\mathbb{R}^{3}$. J. Differential Equations 184 (2002), 587-619.

[14] T. Kobayashi and Y. Shibata. Decay estimates of solutions for the equations of motion of compressible viscous and heat-conductive gases in an exterior domain in $\mathbb{R}^{3}$. Comm. Math. Phys. 200 (1999), 621-659.

[15] A. Matsumura. An energy method for the equations of motion of compressible viscous and heat-conductive fluids, University of Wisconsin-Madison, MRC Technical Summary Report \# 2194 (1981), pp. 1-16.

[16] A. Matsumura and T. Nishida. The initial value problem for the equations of motion of compressible viscous and heat-conductive fluids. Proc. Japan Acad. Ser. A 55 (1979), 337-342.

[17] A. Matsumura and T. Nishida. Initial boundary value problem for the equations of motion of general fluids. Comput. Meth. Appl. Sci. Eng. V (1982), 389-406.

[18] A. Matsumura and T. Nishida. Initial boundary value problems for the equations of motion of compressible viscous and heat-conductive fluids. Comm. Math. Phys. 89 (1983), 445-464. 
[19] H. Sohr. The Navier-Stokes Equations. An Elementary Functional Analytic Approach. Birkhäuser, Basel, 2001.

Reika Aoyama

Graduate School of Mathematics

Kyushu University

Fukuoka, 819-0395

Japan

(E-mail:r-aoyama@kyudai.jp) 